\title{
Results for Phase I of the IAEA Coordinated Research Program on HTGR Uncertainties
}

Friederike Bostelmann

Gerhard Strydom

Su Jong Yoon

January 2015

The INL is a

U.S. Department of Energy

National Laboratory

operated by

Battelle Energy Alliance

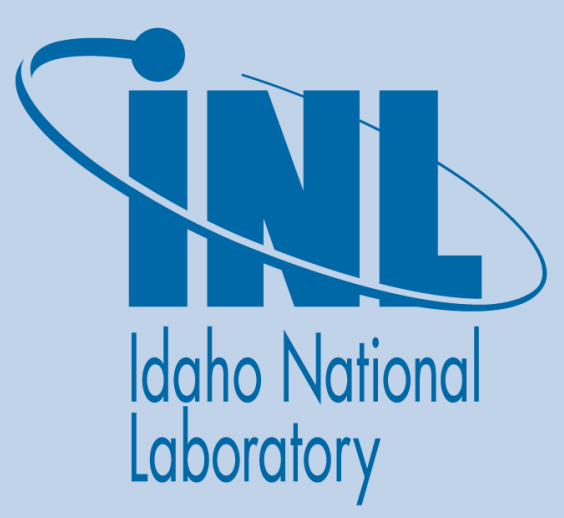

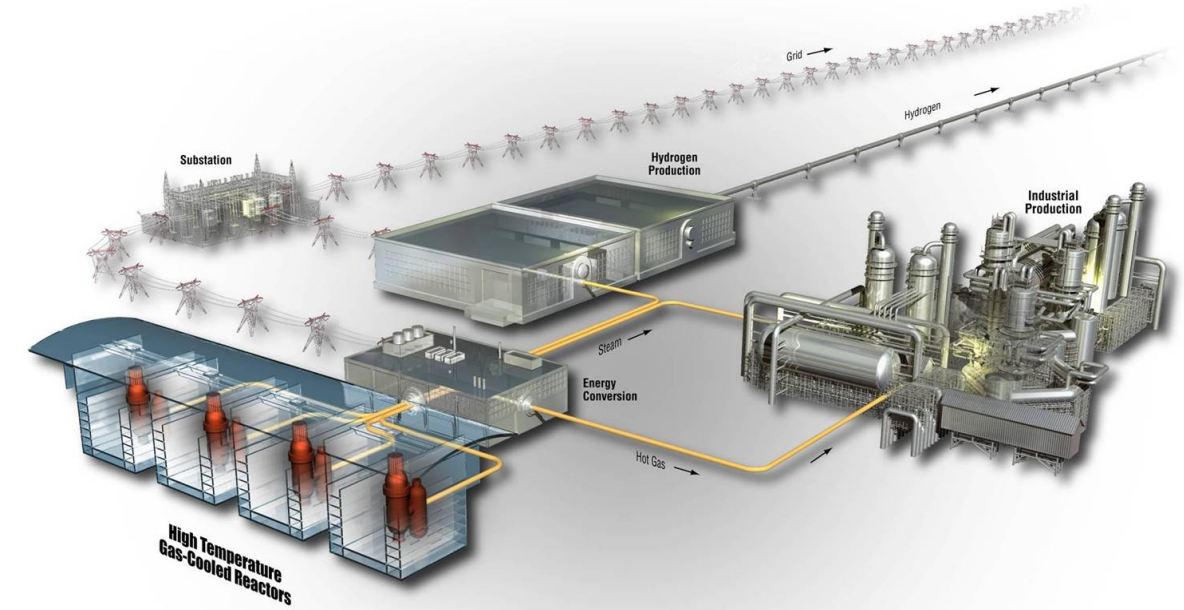

The INL is a U.S. Department of Energy National Laboratory Operated by Battelle Energy Alliance 


\section{DISCLAIMER}

This information was prepared as an account of work sponsored by an agency of the U.S. Government. Neither the U.S. Government nor any agency thereof, nor any of their employees, makes any warranty, expressed or implied, or assumes any legal liability or responsibility for the accuracy, completeness, or usefulness, of any information, apparatus, product, or process disclosed, or represents that its use would not infringe privately owned rights. References herein to any specific commercial product, process, or service by trade name, trade mark, manufacturer, or otherwise, does not necessarily constitute or imply its endorsement, recommendation, or favoring by the U.S. Government or any agency thereof. The views and opinions of authors expressed herein do not necessarily state or reflect those of the U.S. Government or any agency thereof. 
INL/EXT-14-32944

Revision 1

\title{
Results for Phase I of the IAEA Coordinated Research Program on HTGR Uncertainties
}

\author{
Friederike Bostelmann \\ Gerhard Strydom \\ Su Jong Yoon
}

January 2015

\begin{abstract}
Idaho National Laboratory
ART Program

Idaho Falls, Idaho 83415
\end{abstract}

http://www.inl.gov

Prepared for the

U.S. Department of Energy

Office of Nuclear Energy

Under DOE Idaho Operations Office

Contract DE-AC07-05ID14517 



\title{
ART Program
}

\section{Results for Phase I of the IAEA Coordinated Research Program on HTGR Uncertainties}

\author{
INL/EXT-14-32944 \\ Revision 1 \\ January 2015
}

Prepared by:
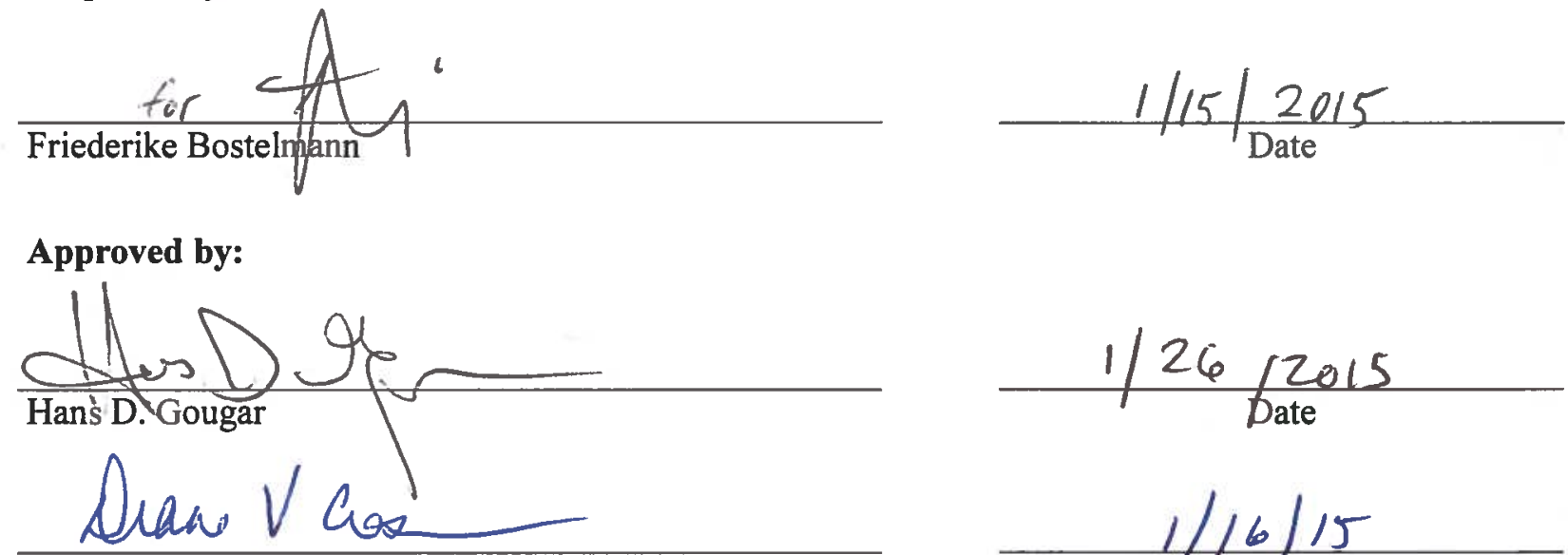

Diane V. Croson

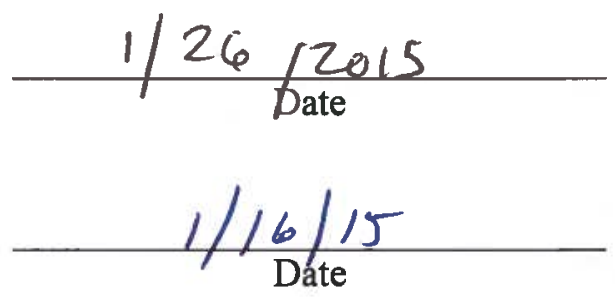

INL ART TDO Deputy Director
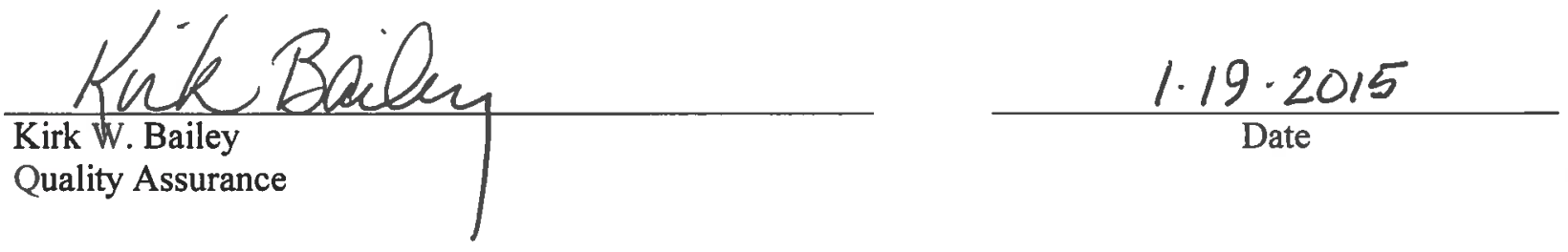



\section{SUMMARY}

Best Estimate Plus Uncertainty analysis of reactors is not only broadly accepted today, but in many cases is replacing the traditional conservative (stacked uncertainty) method for safety and licensing analysis. The use of a more fundamental methodology is also consistent with the reliable high fidelity physics models and robust, efficient, and accurate codes available today. To facilitate uncertainty analysis applications a comprehensive approach and methodology must be developed and applied. High Temperature Gas-cooled Reactors (HTGR) have their own peculiarities, including coated particle design, large graphite quantities, different materials, and high temperatures that require simulation techniques not utilized in LWR analysis. Therefore, in 2013 the IAEA launched a Coordinated Research Project (CRP) on the HTGR Uncertainty Analysis in Modeling (UAM) to study uncertainty propagation, specifically in the HTGR analysis chain. Two benchmark problems are defined, with the prismatic design represented by the General Atomics (GA) MHTGR-350 and a $250 \mathrm{MW}$ modular pebble bed design similar to the HTR-PM (INET, China).

This report summarizes the contributions of the HTGR Methods Simulation group at Idaho National Laboratory (INL) to the CRP through Fiscal Year 2014. The activities at INL have been focused in this period on finalizing the problem specifications for the prismatic design, as well as providing reference solutions for the exercises defined for Phase I. An overview is provided of the HTGR UAM objectives and scope, and the specifications for Exercises I-1, I-2, I-3, and I-4 are also included here for completeness. The main focus of the report is the compilation and discussion of reference results for Phase I (i.e., for input parameters at their nominal or best-estimate values), which is defined as the first step of the uncertainty quantification process. These reference results can be used by other CRP participants for comparison with other codes or their own reference results. The Monte Carlo modeling comparison of the experimental Very High Temperature Test Reactor Critical (VHTRC) facility is also presented. Reference results were obtained for the neutronics stand-alone cases (Exercise I-1 and Exercise I-2) using the latest version of the Monte Carlo code Serpent, and comparisons were performed with the Monte Carlo code SCALE/KENO-VI. For the thermal-fluids stand-alone cases (Exercise I-3 and I-4) the commercial CFD code CFX-13 was utilized to obtain reference results that can be compared with lower fidelity tools. 


\section{ACKNOWLEDGEMENTS}

The authors would like to thank Dr. Javier Ortensi (INL) and Hans-Ruediger Hammer (Texas A\&M) for the use of their Serpent Monte Carlo model for the VHTRC facility as the starting point for our model development. The frequent assistance of Dr. Ortensi with the fine art of neutron transport and the technical review of this document is also gratefully acknowledged. 


\section{CONTENTS}

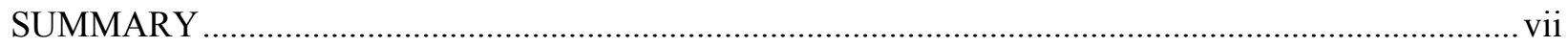

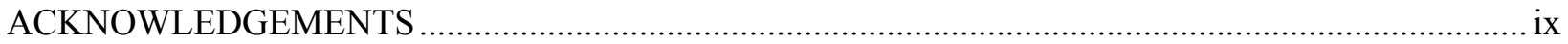

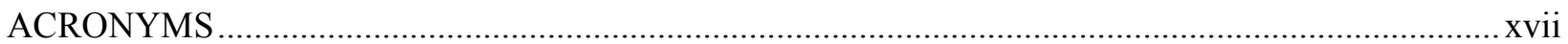

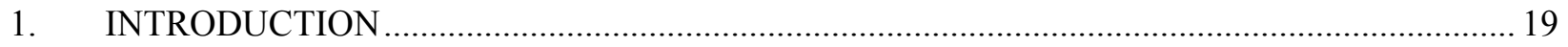

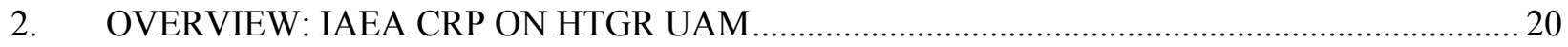

2.1 Phase I Exercises I-1 and I-2: Local Neutronics ................................................................ 21

2.2 Phase I Exercises I-3 and I-4: Local Thermal-Hydraulics .............................................. 21

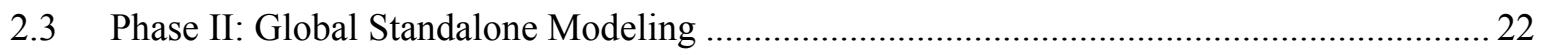

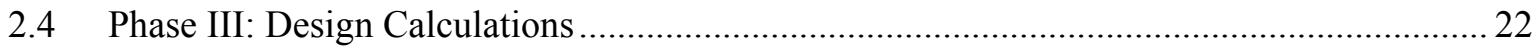

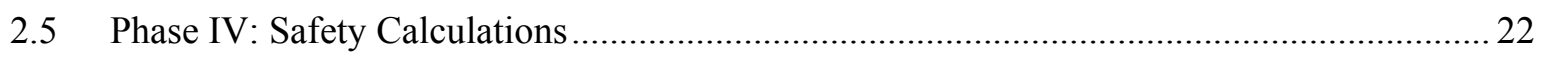

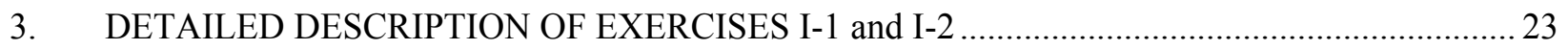

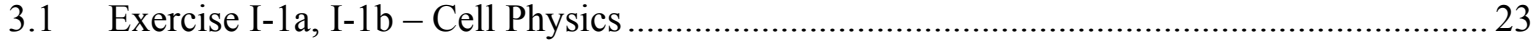

3.2 Exercise I-2a/I-2b: Fresh/Depleted Single Fuel Block Lattice Calculations ........................ 26

3.3 Exercise I-2c: Super-Cell (Mini-Core) Lattice Calculation ................................................. 29

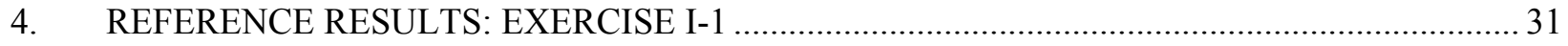

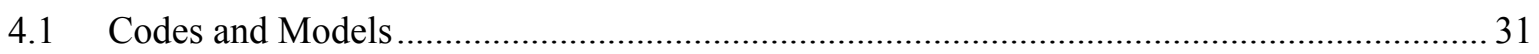

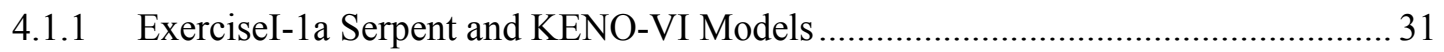

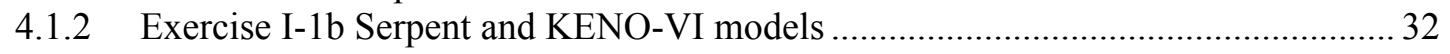

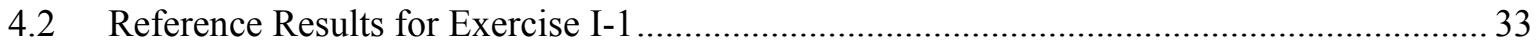

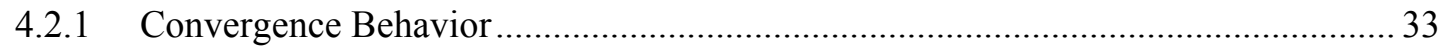

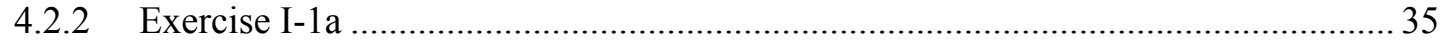

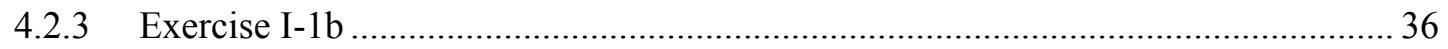

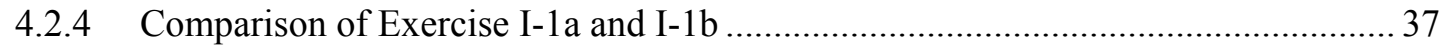

4.2.5 Influence of the Graphite Thermal Scattering Library ............................................. 40

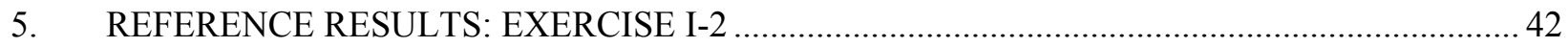

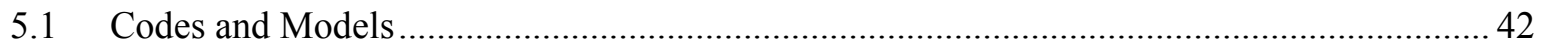

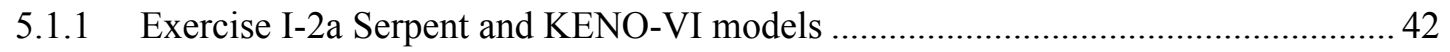

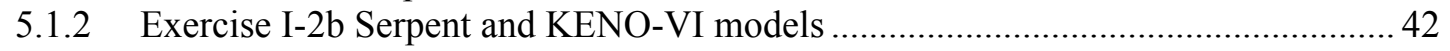

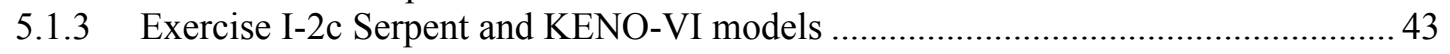

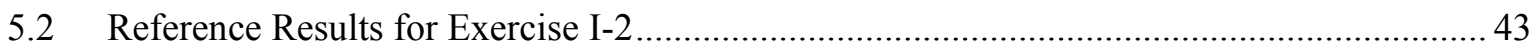

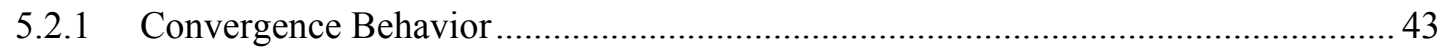

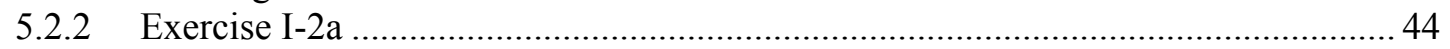

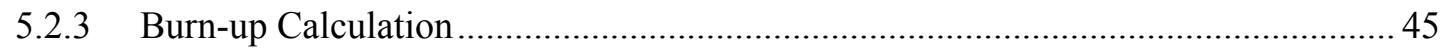

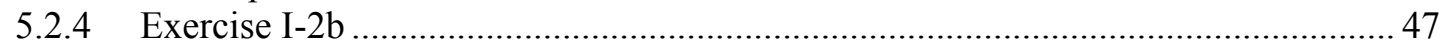

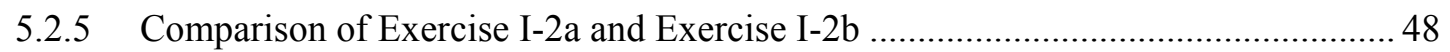

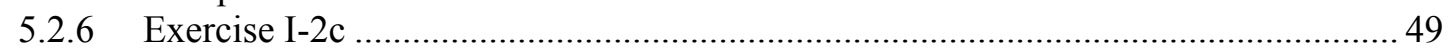

5.3 Concluding Remarks: Exercise I-1 and Exercise I-2 …............................................... 50

6. EXPERIMENTAL VALIDATION RESULTS: THE VHTRC FACILITY ................................ 52 


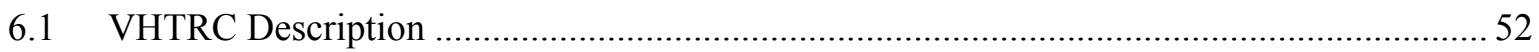

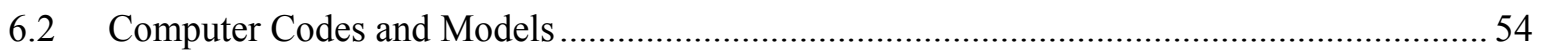

6.2.1 Serpent Model with a Random Particle Distribution ................................................. 54

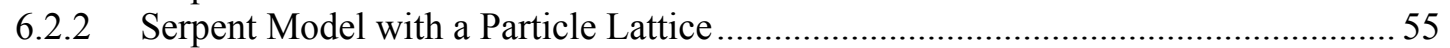

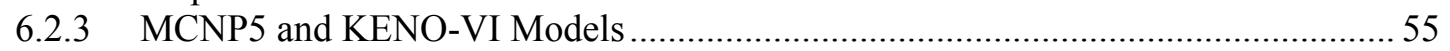

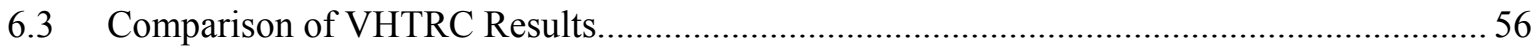

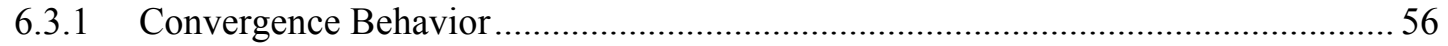

6.3.2 Influence of the KENO-VI/DOUBLEHET Cell Treatment...................................... 57

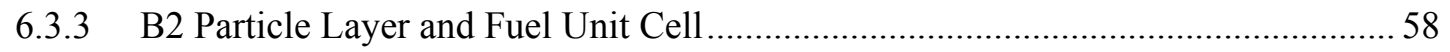

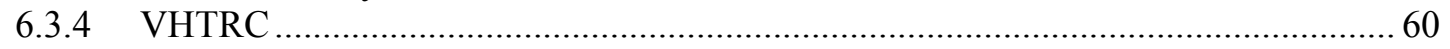

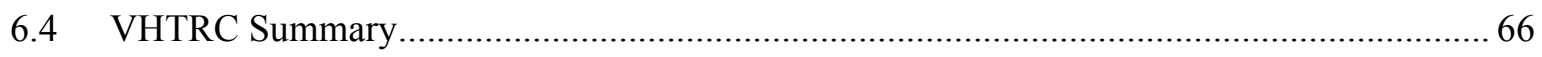

7. EXERCISES I-3 AND I-4: LOCAL THERMAL-HYDRAULIC COUPLING ............................. 67

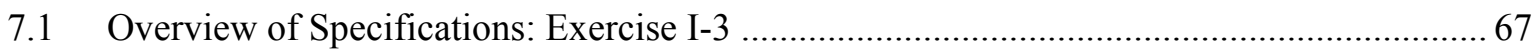

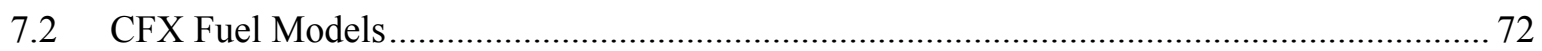

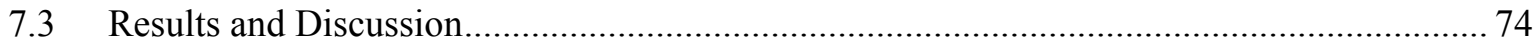

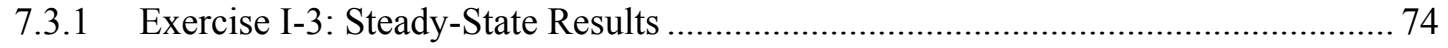

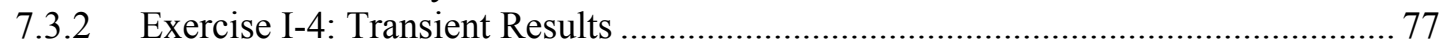

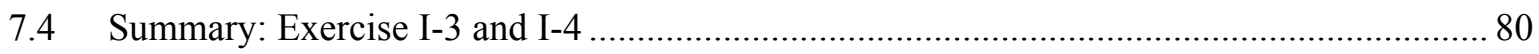

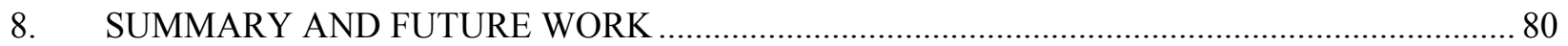

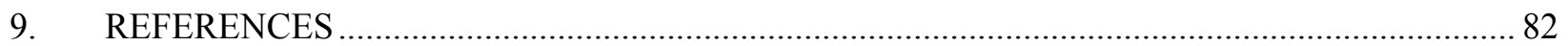

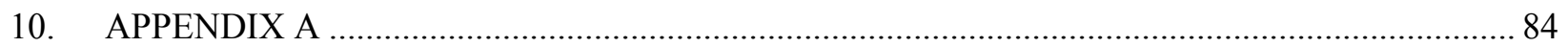

10.1 SCALE/NEWT Criticality Calculations with 238 Groups ................................................ 84

10.2 REACTIVITY-EQUIVALENT Physical Transformation of the MHTGR-350.................. 85

10.3 SCALE/KENO Calculations Using the 56-Group Library ................................................ 86

10.4 SCALE/KENO and SCALE/NEWT Calculations Using an ORNL 81-Group

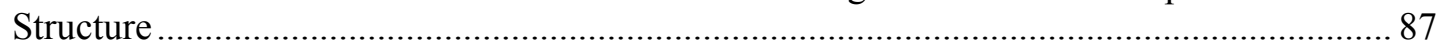

\section{FIGURES}

Figure 1. Single MHTGR fuel compact unit cell for Exercise I-1a and Exercise I-1b...........................23

Figure 2. MHTGR-350 lattice cell for Exercise I-2a (fresh single block)............................................26

Figure 3. MHTGR-350 lattice cell for Exercise I-2b (depleted single block). .......................................2

Figure 4. MHTGR-350 super-cell centered at block 26..................................................................... 30

Figure 5. Simplified representation of the Exercise I-2c super-cell....................................................... 30

Figure 6. Cross-sectional view of the Serpent model for Exercise I-1b with randomly distributed

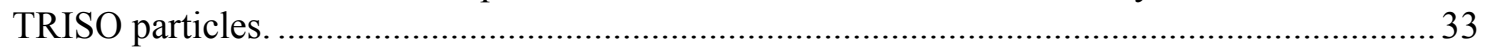

Figure 7. Cross-sectional view of the Serpent model for Exercise I-1b with uniformly distributed TRISO particles. 
Figure 8. Exercise I-1a multiplication factor as a function of neutron cycles $(50,000$ neutrons/cycle).

Figure 9. Exercise I-1b multiplication factor as a function of neutron cycles $(50,000$ neutrons/cycle).

Figure 10. Exercise I-1 a/b Shannon entropy of the fission source distribution as a function of neutron cycles (50,000 neutrons/cycle).

Figure 11. Normalized neutron flux in the fuel compact unit cell (ExerciseI-1a and I-1b)...................... 38

Figure 12. Resonances in the neutron flux of the fuel compact unit cell (extract from Figure 11)............39

Figure 13. Capture reaction rate of ${ }^{238} \mathrm{U}$ in the CZP state (ExerciseI-1a and I-1b)................................... 39

Figure 14. Normalized neutron flux in the unit cell of Exercise I-1b with modified moderator-fuel-ratio.

Figure 15. Exercise I-2a/b multiplication factor as a function of neutron cycles $(50,000$ neutrons/cycle).

Figure 16. Exercise I-2c multiplication factor as a function of neutron cycles $(50,000$ neutrons/cycle).

Figure 17. Exercise I-2 Shannon entropy of the fission source distribution as a function of neutron cycles $(50,000$ neutrons/cycle).

Figure 18. Development of the multiplication factor in a burn-up calculation with and without burnable poison (BP). 46

Figure 19. Development of the ${ }^{10} \mathrm{~B}$ number density during the burn-up calculation. .............................. 46

Figure 20. Neutron flux in the fresh fuel block (Exercise I-2a) with and without BP compacts...............47

Figure 21. Normalized thermal flux in radial direction (Exercise I-2a and Exercise I-2b).................... 48

Figure 22. Normalized neutron flux (Exercise I-2a and Exercise I-2b)................................................ 49

Figure 23. Normalized neutron flux (Exercise I-1b and Exercise I-2a/c) ...........................................50

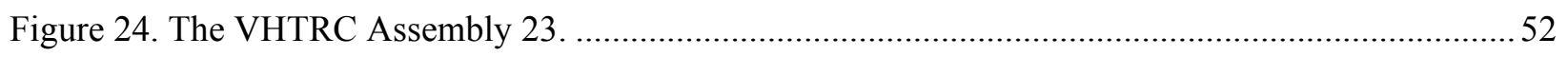

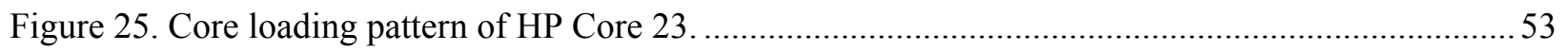

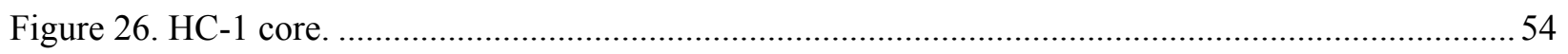

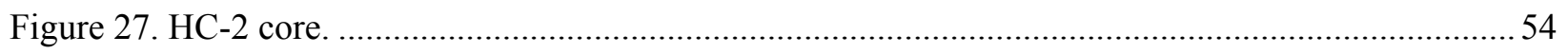

Figure 28. Cross-sectional view of B2 fuel unit cell with a particle lattice (one particle layer)................55

Figure 29. Cross-sectional view of a B2 fuel unit cell with a random particle distribution........................55

Figure 30. Vertical cross section of a B2 fuel unit cell with a particle lattice (not to scale).....................56

Figure 31. Vertical cross section of a B2 fuel unit cell with a random particle distribution (not to scale).

Figure 32. Multiplication factor as a function of neutron cycles with 50,000 neutrons/cycle for the B2 unit cell and the VHTRC HP core at $25.5^{\circ} \mathrm{C}$.

Figure 33. Shannon entropy of the fission source distribution as a function of neutron cycles with 50,000 neutrons/cycle for the B2 unit cell and the VHTRC HP core at $25.5^{\circ} \mathrm{C}$. 
Figure 34. Difference $(\mathrm{mb})$ of the microscopic capture cross section of carbon at $293.6 \mathrm{~K}$ between the ENDF-B-VII.1 and ENDF-B-VII.0 library.

Figure 35. Normalized neutron flux in the B2 fuel unit cell and VHTRC HP core at $25.5^{\circ} \mathrm{C} \ldots \ldots \ldots \ldots \ldots \ldots . . . . . . .61$

Figure 36. Comparison of Serpent 2 and experimental VHTRC multiplication factors.

Figure 37. Comparison of SCALE/KENO-VI CE, Serpent and experimental VHTRC multiplication factors.

Figure 38. Comparison of the SCALE/KENO-VI MG and experiemntal VHTRC multiplication factors.

Figure 39. Difference (pcm) between the SCALE/KENO-VI and the reference Serpent random (ENDF-B-VII.0) multiplication factor of the VHTRC.

Figure 40. Comparison of the Serpent 2 (ENDF-B-VII.1) and MVP-II (JENDL-4.0) VHTRC multiplication factors.

Figure 41. Differences (pcm) between VHTRC multiplication factors of the CRP model and variations including gaps and different material compositions. 66

Figure 42. MHTGR-350 hexagonal and triangular unit cell representations...........................................6 68

Figure 43. MHTGR-350 triangular unit cells for Exercise I-3a/4a and Exercise I-3b/4b........................ 68

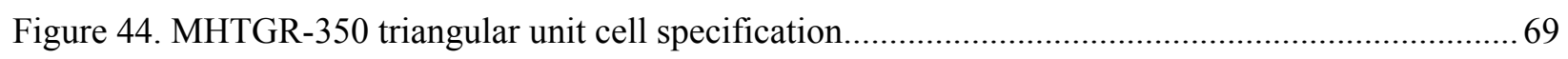

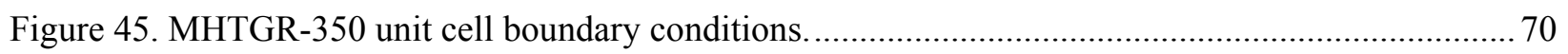

Figure 46. Cross-sectional view of a fuel compact (micrograph). The varying radii of the particles indicate the variable location of the randomly distributed particles relative to the cut-plane. 73

Figure 47. Homogeneous unit-cell model of MHTGR-350 fuel........................................................... 73

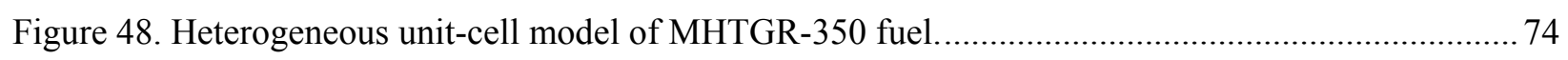

Figure 49. Temperature $(\mathrm{K})$ distribution of the steady-state homogeneous model (Exercise I-3a)........... 75

Figure 50. Temperature $(\mathrm{K})$ distribution of the steady-state heterogeneous model (Exercise I-3b)..........75

Figure 51. Comparison of temperature $(\mathrm{K})$ profiles between homogeneous and heterogeneous models.

Figure 52. Maximum and volume-averaged temperatures $(\mathrm{K})$ of homogeneous model in a transient simulation

Figure 53. The maximum temperature $(\mathrm{K})$ profile of heterogeneous model in a transient simulation.

Figure 54. The volume-averaged temperature $(\mathrm{K})$ profile of heterogeneous model in a transient simulation.

Figure 55. Comparison of maximum temperatures between the homogeneous and heterogeneous model in the transient simulation.

Figure 56. Normalized neutron flux spectrum of the reference heterogeneous Serpent calculation and the homogeneous RPT method (Exercise I-1b, HFP). 


\section{TABLES}

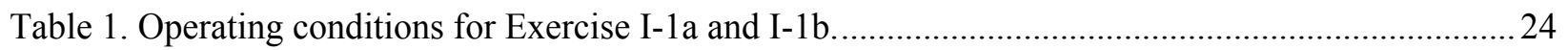

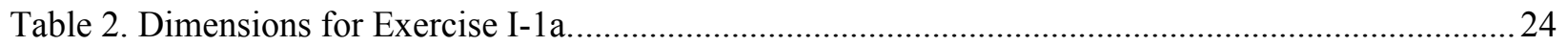

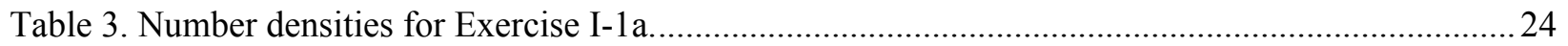

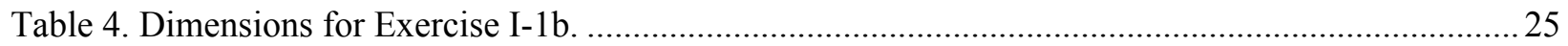

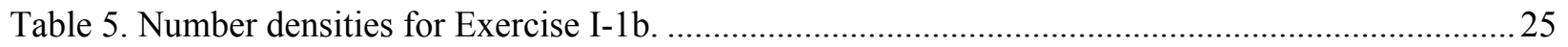

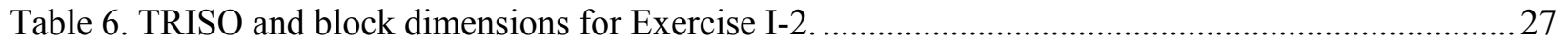

Table 7. Number densities for the fresh fuel block (Exercise I-2a) .....................................................28

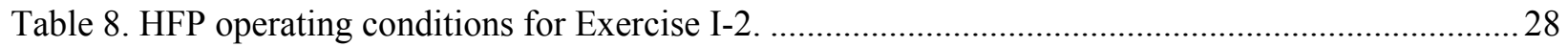

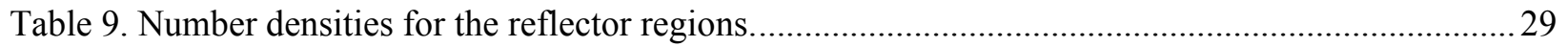

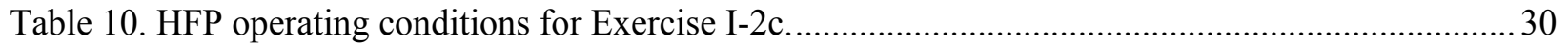

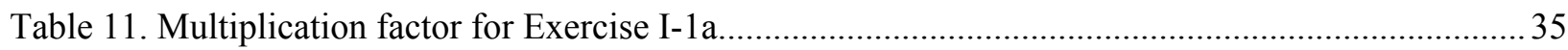

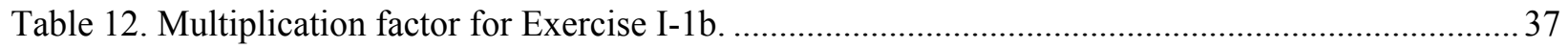

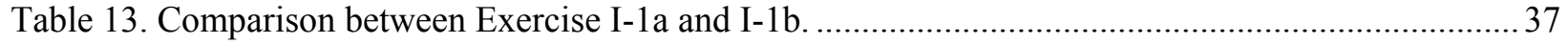

Table 14. Comparison of three cases of Exercise I-1b (random particle distribution). .............................41

Table 15. Comparison of three cases of Exercise I-2a (random particle distribution).............................41

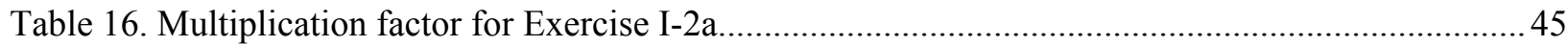

Table 17. Multiplication factor for Exercise I-2b. ......................................................................... 48

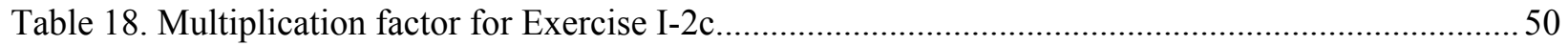

Table 19. Comparison of different KENO-VI/DOUBLEHET cell treatments.......................................58

Table 20. Multiplication factor of a B2 fuel unit cell. ............................................................................ 59

Table 21. Exercise I-3 and I-4 input parameters - nominal values....................................................... 70

Table 22. Thermo-physical properties of $\mathrm{H}-451$ graphite..................................................................... 71

Table 23. Thermo-physical properties of pyrolytic carbon layer............................................................ 71

Table 24. Thermo-physical properties of silicon carbon layer. …........................................................... 71

Table 25. Effective thermo-physical properties of fuel compact........................................................... 71

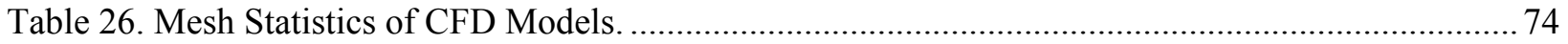

Table 27. Volume-averaged and maximum temperature (K) of the homogeneous model........................ 76

Table 28. Volume-averaged and maximum temperature $(\mathrm{K})$ of the heterogeneous model ....................... 76

Table 29. Comparison of the NEWT and SCALE 6.1.2 KENO MG (238 groups) multiplication

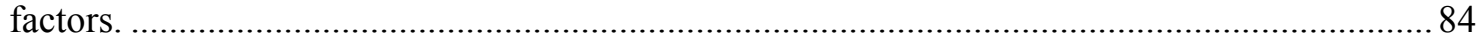

Table 30. RPT values of the fuel compact unit cell (Exercise I-1b)..................................................... 85

Table 31. RPT $\mathrm{k}_{\infty}$ of Exercise I-2a/c compared to the corresponding Serpent calculation........................ 86

Table 32. Multiplication factor of SCALE/KENO $6.2 \mathrm{~b} 3$ calculations with 56 energy groups compared to the respective 238 -group calculations. 
Table 33. Multiplication factor of SCALE/NEWT 6.1.2 calculations with 81 energy groups compared to the respective 238-group calculations.

Table 34. Multiplication factor of SCALE/KENO 6.1.2 calculations with 81 energy groups compared to the respective 238-group calculations. 


\section{ACRONYMS}

BISO Bi-Structural Isotropic (fuel particles)

BP Burnable Poison

CE Continuous Energy

CFD Computational Fluid Dynamics

CRP Coordinated Research Program

CZP Cold Zero Power

DBRC Doppler-Broadening Rejection Correction

DLOFC Depressurized Loss of Forced Cooling

ENDF Evaluated Nuclear Data File

GCR Gas-Cooled Reactor

HFP Hot Full Power

HPC High Performance Cluster

HTGR High Temperature Gas-Cooled Reactor

HTTR High Temperature Engineering Test Reactor

IAEA International Atomic Energy Agency

ICAPP International Congress on Advances in Nuclear Power Plants

IHRP International Handbook of Reactor Physics Experiments

INL Idaho National Laboratory

IPyC Inner Pyrolytic Carbon

JAEA Japan Atomic Energy Agency

LBP Lumped Burnable Poison

LWR Light Water Reactor

MCNP Monte Carlo N-Particle

MG Multi-group

MHTGR Modular High Temperature Gas-cooled Reactor

MPI Message Passing Interface

NDL Nuclear Data Libraries

NEA Nuclear Energy Agency

NRF National Research Foundation of Korea

O Output

OECD Organization for Economic Cooperation and Development

OMP Open Multi-Processing

OPyC outer pyrolytic carbon 
ORNL Oak Ridge National Laboratory

PCM per cent mille

PDF Probability Distribution Functions

RIT Reactivity Induced Transient

RPT Reactivity-Equivalent Physical Transformation

SA Sensitivity Analysis

SCALE Standardized Computer Analyses for Licensing Evaluation

SiC Silicon Carbide

SUSA Software System for Uncertainty and Sensitivity Analyses

TRISO Tri-Structural Isotropic

TWG Technical Working Group

UA Uncertainty Analysis

UAM Uncertainty in Analysis Modeling

UCO Uranium Oxycarbide

UP Uncertainty Parameters

$\mathrm{V} \& \mathrm{~V} \quad$ Verification and Validation

VHTRC Very High Temperature Test Reactor Critical 


\section{Results for Phase I of the IAEA CRP on HTGR Uncertainties}

\section{INTRODUCTION}

The continued development of High Temperature Gas Cooled Reactors (HTGRs) requires verification of HTGR design and safety features with reliable high fidelity physics models and robust, efficient, and accurate codes. The predictive capability of coupled neutronics/thermal-hydraulics and depletion simulations for reactor design and safety analysis can be assessed with sensitivity analysis (SA) and uncertainty analysis (UA) methods. Uncertainty originates from errors in physical data, manufacturing uncertainties, modeling, and computational algorithms. SA is helpful for ranking the various sources of uncertainty and error in the results of core analyses. SA and UA are required to address cost, safety, and licensing needs and should be applied to all aspects of reactor multi-physics simulation. SA and UA can guide experimental, modeling, and algorithm research and development. Current SA and UA rely either on derivative-based methods such as stochastic sampling methods or generalized perturbation theory to obtain sensitivity coefficients. Neither approach addresses all needs.

To benefit from recent advances in modeling and simulation and the availability of new covariance data (nuclear data uncertainties), extensive sensitivity and uncertainty studies are needed for quantification of the impact of different sources of uncertainties on the design and safety parameters of HTGRs. Only a parallel effort in advanced simulation and in nuclear data improvement will be able to provide designers with more robust and well-validated calculation tools to meet design target accuracies.

In February 2009, the Technical Working Group on Gas-Cooled Reactors (TWG-GCR) of the International Atomic Energy Agency (IAEA) recommended the proposed Coordinated Research Program (CRP) on the HTGR Uncertainty Analysis in Modeling (UAM) be implemented. This CRP is a continuation of the previous IAEA and Organization for Economic Co-operation and Development (OECD)/Nuclear Energy Agency (NEA) international activities on Verification and Validation (V\&V) of available analytical capabilities for HTGR simulation for design and safety evaluations. ${ }^{1,2,3}$ Within the framework of these activities different numerical and experimental benchmark problems were performed and insight was gained about specific physics phenomena and the adequacy of analysis methods.

The CRP benefits from interactions with the currently ongoing OECD/NEA Light Water Reactor (LWR) UAM benchmark activity ${ }^{4}$ by taking into consideration the peculiarities of HTGR designs and simulation requirements. Since the prismatic design specification is based directly on the OECD/NEA Modular High Temperature Gas-cooled Reactor (MHTGR)-350 MW benchmark, ${ }^{5}$ participants in both activities can leverage their core models developed for the OECD/NEA benchmark for this CRP benchmark with only minor changes.

In Section 2, an overview is provided on the objectives and layout of the CRP on HTGR UAM. A more detailed description of the four exercises defined for Phase I are included in Section 3 for completeness, based on the latest draft released to the CRP participants (November 2014). ${ }^{6}$ The Monte Carlo results obtained for Exercises I-1 and I-2 are discussed in Sections 4 and 5, respectively. The experimental validation results of the Very High Temperature Reactor Critical Assembly (VHTRC) facility are discussed in Section 6, and the CFX results obtained for Exercises I-3 and I-4 are included in Section 7. The results of several additional studies performed during the development of the Phase I specifications are included in Section 10. In addition to the status and results reported here, the first TSUNAMI, SAMPLER, and SUSA results have also been obtained for most of these exercises. These uncertainty quantification results will be summarized in a separate report at a later stage. 


\section{OVERVIEW: IAEA CRP ON HTGR UAM}

SA and UA methods need to be considered as an integral part of the development of coupled code methods. Of particular importance are innovative methods that address nonlinearity, can predict the probability distributions in output parameters, can treat discrete events, and handle simultaneously large input data and response fields in a computationally efficient manner.

In the IAEA CRP on HTGR UAM different SA and UA methods will be compared, further developed, and their values assessed, including the validation of the methodologies for uncertainty propagation in HTGR modeling. The uncertainty propagation will be estimated through the whole simulation process on a unified benchmark framework to provide credible coupled code predictions with defensible uncertainty estimations of safety margins at the full core/system level. The proposed program will help to utilize the community of experts created during the previous IAEA and OECD HTGR-related activities and expand it by combining expertise in physics (neutronics and thermal-hydraulics) and in SA and UA. The CRP will allow these experts to not only compare and assess the current SA and UA methods on representative applications, but will also stimulate further development of efficient and powerful SA and UA methods suitable for complex coupled code simulations. The CRP will also help to formulate recommendations and guidelines on how to utilize advanced and optimized SA and UA methods in "best estimate" reactor simulations in HTGR licensing practices.

The objective is to determine the uncertainty in HTGR calculations at all stages of coupled reactor physics/thermal hydraulics and depletion calculations. To accomplish this objective, a benchmark platform for uncertainty analysis in best-estimate coupled code calculations for design and safety analysis of HTGRs will be defined and utilized. The full chain of uncertainty propagation from basic data, engineering uncertainties, across different scales (multi-scale), and physics phenomena (multi-physics) will be tested on a number of benchmark exercises with maximum utilization of the available experimental data, published benchmark results, and released design details. Two main HTGR types (prismatic and pebble bed HTGRs) are selected based on previous benchmark experiences and available data. In principle the sources of Input (I) uncertainties in computer code simulations can be identified as:

- Input data uncertainties

- Model limitations

- Approximations in the numerical solution

- Nodalization

- Homogenization approaches

- Imperfect knowledge of boundary and initial conditions.

For each exercise it is important to identify which new input uncertainties are taken into account and which input uncertainties are propagated from the previous exercise. In Phase I of the benchmark, the input uncertainties are specified as follows: best-estimate values for input parameters supplemented by the variance-covariance matrices (utilized for cross-section uncertainties), and for other input uncertainties, probability distribution functions (PDF) and associated parameters.

Other important parameters to be defined are the Output $(\mathrm{O})$ uncertainties and propagated Uncertainty parameters (U) for each exercise. This task is directly related to the objective of each exercise. The Output uncertainties are defined for specified output parameters for each exercise to test (evaluate) the utilized uncertainty method. The propagated Uncertainty parameters are output parameters selected to be propagated further through follow-up exercises to calculate the overall resulting uncertainty. The aim is to propagate as many uncertainties as feasible and as realistic to the subsequent coupled calculations.

An overview of the phases and exercises defined for the benchmark is provided here. More detail is provided on the Phase I exercises in Section 3. 


\subsection{Phase I Exercises I-1 and I-2: Local Neutronics}

These exercises are focused on the derivation of the multi-group and few-group microscopic cross-section libraries. The objective is to address the uncertainties due to the basic nuclear data as well as the impact of processing the nuclear and covariance data, selection of multi-group structure, and double heterogeneity or self-shielding treatment. The intention is to propagate the uncertainties in evaluated Nuclear Data Libraries (microscopic point-wise cross sections) into multi-group microscopic cross sections, and to propagate the uncertainties from the multi-group microscopic cross sections into the few-group cross sections for use in Phase II. Two exercises are defined to capture the local neutronics effects.

- Exercise I-1 (I-1a, I-1b) - Cell Physics: Derivation of the multi-group microscopic cross-section libraries.

A basic unit cell is defined for Exercise 1 based on the MHTGR-350 design parameters. Two sub-cases are included: Exercise I-1a specifies a homogeneous fuel region of homogenized Tri-Structural Isotropic (TRISO) fuel particles and matrix graphite, whereas Exercise I-1b requires the explicit modeling of the TRISO fuel particles to investigate their self-shielding effect on the multi-group microscopic cross-sections.

- Exercise 1-2 (I-2a, I-2b, I-2c) - Lattice Physics: Derivation of the few-group macroscopic cross-section libraries

The lattice level calculation for the prismatic MHTGR-350 is defined for two sub-cases to investigate the effect of neighboring spectra on the few-group cross sections, since HTGR designs are typically coupled over larger distances than LWR designs. Exercise I-2a requires a lattice calculation to be performed on a single fuel block at Hot Full Power (HFP) conditions, while Exercise I-2b specifies the same problem at $100 \mathrm{MWd} / \mathrm{kg} \mathrm{U}$ burn-up.

Exercise I-2c adds the effects of the neighboring domain by performing a lattice calculation on a super cell (or mini-core), which consists of a fresh fuel block surrounded by depleted fuel on one side and graphite reflector blocks on the other side. This calculation is also performed at HFP conditions.

\subsection{Phase I Exercises I-3 and I-4: Local Thermal-Hydraulics}

These exercises focus on the localized stand-alone fuel thermal response. The aim of the stand-alone thermal unit cell calculations is to isolate the effect of material and boundary input uncertainties on very simplified problems, before the same input variations are applied to complex core problems (Phases II-IV). The figures of merit for both exercises are the variation in the unit cell temperature profiles due to input uncertainty variations in the material properties and boundary conditions. No output parameters will be propagated into subsequent exercises or phases, but the same material input uncertainties (also called manufacturer uncertainties) will be specified in the subsequent phases.

\section{- Exercise I-3 (I-3a, I-3b): Stand-alone thermal-hydraulics (normal operation)}

Exercise I-3 requires a steady-state solution for a single fuel compact and coolant channel unit cell with a fixed bulk coolant temperature. Two sub-cases similar to Exercise I-1 are again defined here, taking into account the explicit modeling of heat transfer from the TRISO fuel particles to the matrix graphite. 
- Exercise I-4 (I-4a, I-4b): Stand-alone thermal-hydraulics (power excursion transient)

Exercises I-4a and I-4b use the same unit cell definition as described for Exercise I-3, but a time-dependent power excursion is prescribed, as opposed to a constant steady-state power. The two case variations here are designed to study the effect of additional uncertainties in the explicit modeling of the Uranium Oxycarbide (UCO) TRISO kernels (e.g., variations in the SiC thickness, density and conductivity).

\subsection{Phase II: Global Standalone Modeling}

- Exercise II-1a: Core physics: Criticality (steady-state) stand-alone neutronics calculations

A full-core steady-state neutronics calculation is to be performed using the given fuel number densities and core temperature distributions.

\section{- Exercise II-1b: Core physics: Stand-alone kinetics without feedback}

This exercise involves a full-core calculation with reactivity being added and then returned to normal, but without any temperature feedback. The reactivity-induced transient (RIT) is defined as control rod movement at normal or even slower speed to ensure that the delayed neutrons play a role (no prompt critical). The uncertainties in the kinetic parameters are added in this case.

- Exercise II-2a: Stand-alone thermal-hydraulics focused on core thermal-hydraulic modeling (normal operation)

The conditions at normal operation are considered with only the reactor core modeled and with boundary condition defined for the inlet coolant temperature and pressure and the vessel defined with a constant temperature boundary condition. The reactor power distribution is also specified. Variation in bypass flows and pebble packing fractions are some of the uncertainties to be taken into account.

- Exercise II-2b: Stand-alone thermal-hydraulics focused on core thermal-hydraulic modeling (DLOFC transient)

A Depressurized Loss of Forced Cooling (DLOFC) calculation from full-power conditions is performed. The uncertainties in the steady-state power profile and temperatures from Exercises II-1a and II-2a are input to this calculation. The decay heat uncertainties also need to be addressed.

\subsection{Phase III: Design Calculations}

\section{- Exercise III-1: Coupled Steady-State}

This is the first exercise that requires a coupled calculation focused on the steady-state full power neutronics/thermal-hydraulics core performance. Many uncertainties determined in the previous stand-alone cases will be propagated.

\section{- Exercise III-2: Coupled Depletion}

The depletion is added to the full core coupled neutronics/thermal-hydraulics core calculation and an equilibrium cycle will be calculated. Alternatively, a simplified depletion case with given power history may be evaluated.

\subsection{Phase IV: Safety Calculations}

\section{- Exercise IV-1: Coupled Core Transient}

The coupled core transient with full thermal feedback will be a reactivity induced power excursion due to control rod withdrawal. The feedback effect from the rest of the power conversion unit is to be kept constant or described by a well-defined function. The focus is on the core response only. 


\section{- Exercise IV-2: Coupled System Transient}

The transient of interest is the change in helium inlet coolant temperature with the associated feedback on neutronics, and is focused on the coupled core/thermal-hydraulic system transient performance.

\section{DETAILED DESCRIPTION OF EXERCISES I-1 and I-2 3.1 Exercise I-1a, I-1b - Cell Physics}

These two exercises are focused on the derivation of the multi-group microscopic cross-section libraries for use in the subsequent lattice calculations. The objective is to address the uncertainties due to the basic nuclear data as well as the impact of processing the nuclear and covariance data, selection of multi-group structure, and double heterogeneity or self-shielding treatment. It is possible to propagate the microscopic point-wise cross-sections uncertainties in evaluated Nuclear Data Libraries (NDL) into multi-group microscopic cross-sections used as input by lattice physics codes. The output uncertainties of Exercise I-1 can be used as input uncertainties in Exercise I-2. This exercise is identical in scope and objective to Exercise I-1 (Cell Physics) of the OECD LWR Uncertainty benchmark, ${ }^{4}$ and most details around the use of covariance data are directly applicable.

A representative 2-D "unit cell" for the MHTGR-350 fuel is shown in Figure 1 consisting of the UCO fuel compact, a small gap, and the surrounding H-451 block graphite. This unit cell is derived from the larger fuel block hexagonal geometry, as shown in Figure 2, where each of the blue helium coolant channels remove heat generated by two of the yellow fuel compacts. The only difference between Exercise I-1a and Exercise I-1b is the modeling of the fuel zone: Exercise I-1a specifies a homogeneous fuel region of "smeared-out" TRISO fuel particles and matrix graphite, whereas Exercise I-1b requires the explicit modeling of the TRISO fuel particles to investigate their self-shielding effect on the multi-group microscopic cross sections.

A reflective boundary condition is specified for this problem, since most of the fuel compacts are surrounded by identical unit cells (the exception to this assumption is addressed in the next exercise). One set of unit cell number densities will be defined for two sub-cases: a "fresh" xenon-free Cold Zero Power (CZP) and a HFP core state. These states differ only in the temperature. The data from the two core states can be propagated to the subsequent lattice (Exercise I-2) and core (Exercises II-1a, II-1b, III-1 and III-2) calculations. These two simplified "unit cell" cases will be used to find the major contributors to the multi-group cross-section uncertainties, and possibly to create the multi-group cross-section libraries for use in Exercise I-2. The CZP/HFP operating conditions, dimensions, and number densities for Exercise $\mathrm{I}-1 \mathrm{a}$ and $\mathrm{I}-1 \mathrm{~b}$ are specified in Table 1 to Table 5.

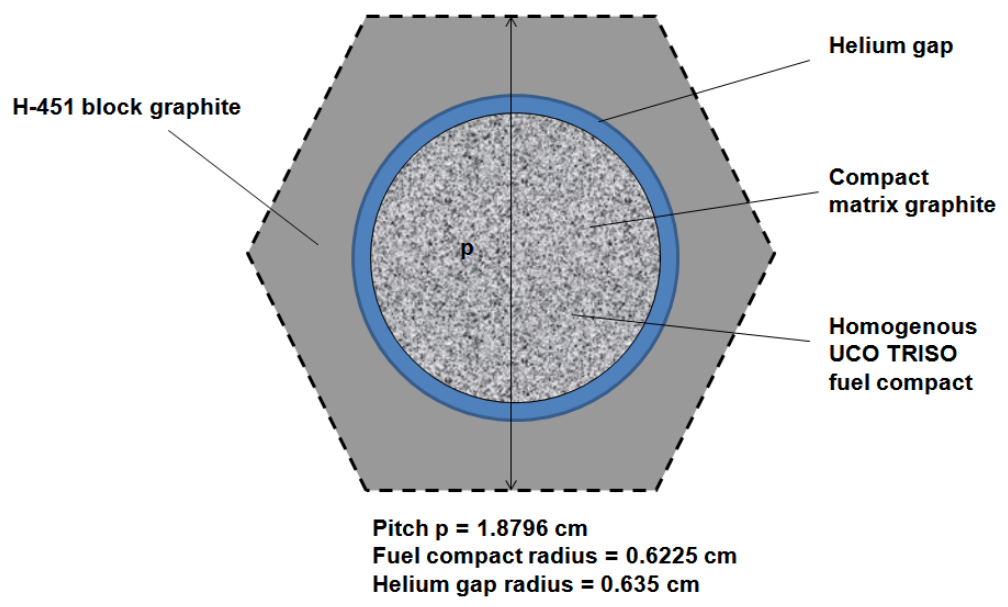

Figure 1. Single MHTGR fuel compact unit cell for Exercise I-1a and Exercise I-1b. 
Table 1. Operating conditions for Exercise I-1a and I-1b.

\begin{tabular}{|l|c|c|}
\hline \multicolumn{1}{|c|}{ Parameter/Reactor Condition } & CZP & HFP \\
\hline Temperature of all material in fuel compact [K] & 600 & 1,200 \\
\hline Temperature of helium in gap [K] & 600 & 1,200 \\
\hline Temperature of H-451 block graphite [K] & 400 & 1,000 \\
\hline
\end{tabular}

Table 2. Dimensions for Exercise I-1a.

\begin{tabular}{|l|c|}
\hline \multicolumn{1}{|c|}{ Parameter } & Dimension $(\mathrm{cm})$ \\
\hline Fuel compact outer radius & 0.6225 \\
\hline Helium gap outer radius & 0.6350 \\
\hline Half unit cell pitch & 0.9398 \\
\hline Fuel compact height & 4.9280 \\
\hline
\end{tabular}

Table 3. Number densities for Exercise I-1a.

\begin{tabular}{|c|c|c|}
\hline \multicolumn{2}{|c|}{ Nuclide } & Number Density $($ atoms $/ \mathrm{b} \cdot \mathrm{cm})$ \\
\hline \multirow[t]{7}{*}{ Homogenized fuel region } & ${ }^{235} \mathrm{U}$ & $1.58 \mathrm{E}-04$ \\
\hline & ${ }^{238} \mathrm{U}$ & $8.47 \mathrm{E}-04$ \\
\hline & ${ }^{16} \mathrm{O}$ & $1.51 \mathrm{E}-03$ \\
\hline & Graphite $^{a}$ & $7.00 \mathrm{E}-02$ \\
\hline & ${ }^{28} \mathrm{Si}$ & $2.85 \mathrm{E}-03$ \\
\hline & ${ }^{29} \mathrm{Si}$ & $1.40 \mathrm{E}-04$ \\
\hline & ${ }^{30} \mathrm{Si}$ & $8.96 \mathrm{E}-05$ \\
\hline Coolant Channel & $4 \mathrm{He}$ & $2.46 \mathrm{E}-05$ \\
\hline H-451 Block Graphite & Graphite $^{\mathrm{b}}$ & $9.28 \mathrm{E}-02$ \\
\hline
\end{tabular}

a Graphite is defined here as natural carbon including thermal scattering data for graphite. In the SCALE code, this is C-graphite, in Serpent/MCNP this is natural carbon with the additional thermal scattering data applied. This definition is applied for all neutronics problems in this benchmark (cf. Section 4.2.5).

b Although it is recognized that the fuel block graphite (H-451 grade) is not the same graphite structure/composition used for the fuel compact graphite, a simplifying assumption is made that both graphite types are identical. 
Table 4. Dimensions for Exercise I- $1 \mathrm{~b}$.

\begin{tabular}{|l|l|c|}
\hline \multicolumn{1}{|c|}{ Parameter } & Dimension (cm) \\
\hline \multirow{4}{*}{$\begin{array}{l}\text { TRISO } \\
\text { fuel particle }\end{array}$} & UCO kernel radius & $2.125 \mathrm{E}-02$ \\
\cline { 2 - 3 } & Porous carbon buffer layer outer radius & $3.125 \mathrm{E}-02$ \\
\cline { 2 - 3 } & Inner PyC outer radius & $3.525 \mathrm{E}-02$ \\
\cline { 2 - 3 } & SiC outer radius & $3.875 \mathrm{E}-02$ \\
\cline { 2 - 3 } & Outer PyC outer radius & $4.275 \mathrm{E}-02$ \\
\hline TRISO Packing Fraction & & 0.35 \\
\hline Fuel compact outer radius & 0.6225 \\
\hline Helium gap outer radius & & 0.6350 \\
\hline Half unit cell pitch & 0.9398 \\
\hline Fuel compact height & 4.9280 \\
\hline
\end{tabular}

Table 5. Number densities for Exercise I-1b.

\begin{tabular}{|c|c|c|c|}
\hline \multicolumn{2}{|c|}{ Number Densities } & Nuclide & $\mathrm{ND}$ (atoms/b-cm) \\
\hline \multirow{11}{*}{$\begin{array}{l}\text { TRISO } \\
\text { fuel particle }\end{array}$} & \multirow{4}{*}{ Kernel } & ${ }^{235} \mathrm{U}$ & $3.67 \mathrm{E}-03$ \\
\hline & & ${ }^{238} \mathrm{U}$ & $1.97 \mathrm{E}-02$ \\
\hline & & ${ }^{16} \mathrm{O}$ & $3.51 \mathrm{E}-02$ \\
\hline & & Graphite & $1.17 \mathrm{E}-02$ \\
\hline & Porous Carbon & Graphite & $5.26 \mathrm{E}-02$ \\
\hline & IPyC & Graphite & $9.53 \mathrm{E}-02$ \\
\hline & \multirow{4}{*}{$\mathrm{SiC}$} & ${ }^{28} \mathrm{Si}$ & $4.43 \mathrm{E}-02$ \\
\hline & & ${ }^{29} \mathrm{Si}$ & $2.17 \mathrm{E}-03$ \\
\hline & & ${ }^{30} \mathrm{Si}$ & $1.39 \mathrm{E}-03$ \\
\hline & & Graphite & $4.79 \mathrm{E}-02$ \\
\hline & OPyC & Graphite & $9.53 \mathrm{E}-02$ \\
\hline \multicolumn{2}{|c|}{ Compact matrix } & Graphite & $7.27 \mathrm{E}-02$ \\
\hline \multicolumn{2}{|c|}{ Coolant Channels } & ${ }^{4} \mathrm{He}$ & $2.46 \mathrm{E}-05$ \\
\hline \multicolumn{2}{|c|}{ H-451 Block Graphite } & Graphite & $9.28 \mathrm{E}-02$ \\
\hline
\end{tabular}




\subsection{Exercise I-2a/l-2b: Fresh/Depleted Single Fuel Block Lattice Calculations}

Exercise I-2 includes the propagation of input uncertainties, as defined below, through lattice physics calculations to target and output uncertainties in evaluated lattice-averaged parameters. The geometry and isotopic data for the simplified single MHTGR-350 hexagonal fuel blocks are shown in Figure 2 and specified in Table 6 and Table 7. The fuel block defined for Exercise I-2a (Figure 2) is representative of fresh fuel and includes six Lumped Burnable Poison (LBP) compacts in the six corners of the block. The fuel block defined for Exercise I-2b (Figure 3) represents a depleted lattice version of Exercise I-2a. The corresponding nuclide densities for the depleted fuel were obtained by a Serpent depletion calculation of the Exercise I-2a fresh block without burnable poison up to $100 \mathrm{MWd} / \mathrm{kg} \mathrm{U}$. A constant power density of $0.1 \mathrm{~kW} / \mathrm{g}$ initial uranium and no downtime was assumed.

This exercise requires the treatment of the double heterogeneity effects (i.e., the self-shielding that occurs both within the fuel and LBP compacts, as well as the effect of multiple compacts present in a single block). (Note that the LBP compact itself consists of a several thousand coated particles of $\mathrm{B}_{4} \mathrm{C}$ with buffer and $\mathrm{PyC}$ coating layers, as indicated in Table 6). The depleted fuel block was specified to not contain burnable poison compacts.

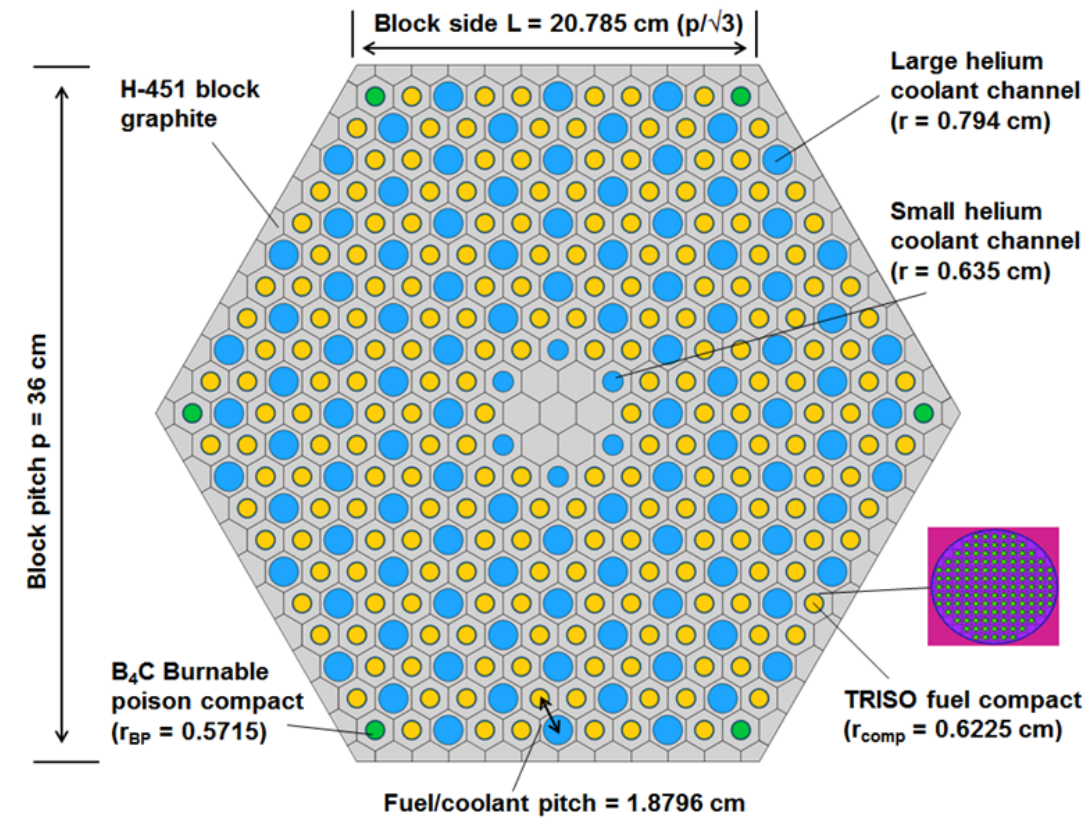

Figure 2. MHTGR-350 lattice cell for Exercise I-2a (fresh single block). 


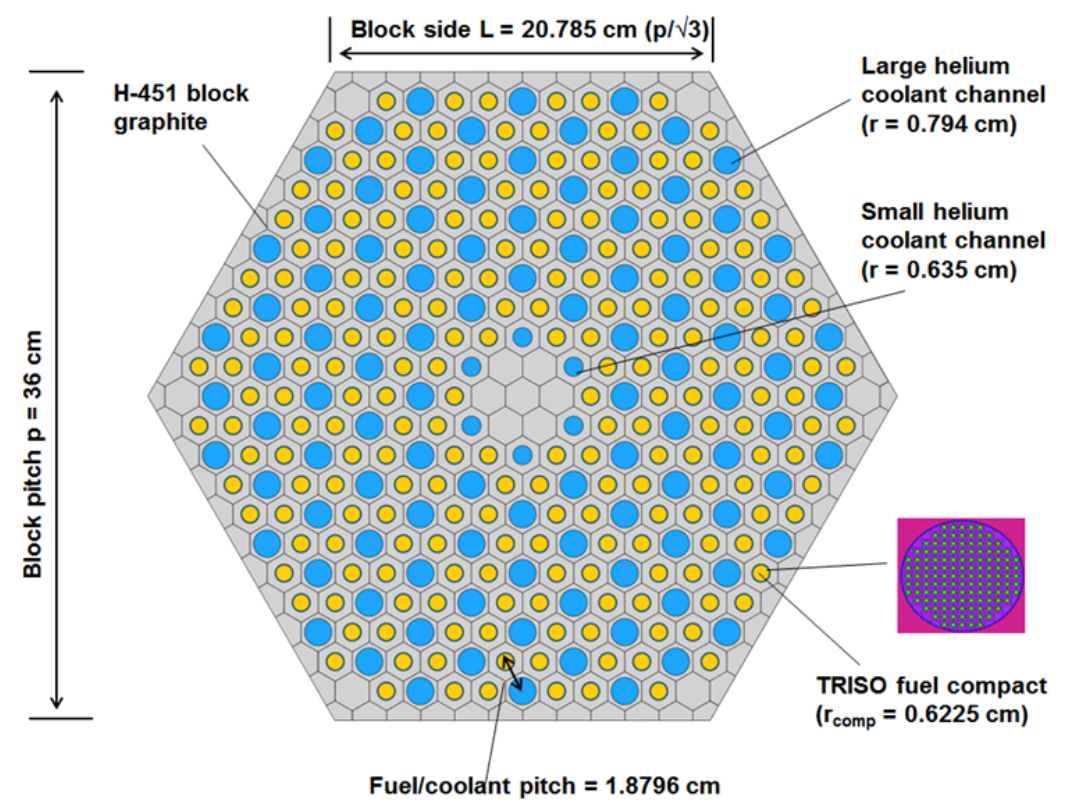

Figure 3. MHTGR-350 lattice cell for Exercise I-2b (depleted single block).

Table 6. TRISO and block dimensions for Exercise I-2.

\begin{tabular}{|l|l|l|c|}
\hline \multicolumn{1}{|c|}{ Parameter } & Dimension & Units \\
\hline \multirow{4}{*}{ TRISO Fuel Particle } & $\mathrm{UC}_{0.5} \mathrm{O}_{1.5}$ kernel radius & $2.125 \mathrm{E}-02$ & $\mathrm{~cm}$ \\
\cline { 2 - 4 } & Porous carbon buffer Outer Radius (OR) & $3.125 \mathrm{E}-02$ & $\mathrm{~cm}$ \\
\cline { 2 - 4 } & IPyC OR & $3.525 \mathrm{E}-02$ & $\mathrm{~cm}$ \\
\cline { 2 - 4 } & SiC OR & $3.875 \mathrm{E}-02$ & $\mathrm{~cm}$ \\
\cline { 2 - 4 } & OPyC OR & $4.275 \mathrm{E}-02$ & $\mathrm{~cm}$ \\
\hline TRISO packing fraction & 0.35 & - \\
\hline Fuel compact radius & 0.6225 & $\mathrm{~cm}$ \\
\hline Gap radius & 0.6350 & $\mathrm{~cm}$ \\
\hline Number of fuel compacts per block & 210 & - \\
\hline \multirow{3}{*}{$\begin{array}{l}\text { Lumped Burnable } \\
\text { Poison Particle }\end{array}$} & Kernel radius & $1.00 \mathrm{E}-02$ & $\mathrm{~cm}$ \\
\cline { 2 - 4 } & Porous carbon buffer OR & $1.18 \mathrm{E}-02$ & $\mathrm{~cm}$ \\
\cline { 2 - 4 } & PyC OR & $1.41 \mathrm{E}-02$ & $\mathrm{~cm}$ \\
\hline Burnable poison particle packing fraction & 0.1090 & - \\
\hline Burnable poison compact radius & 0.5715 & $\mathrm{~cm}$ \\
\hline Large coolant channel radius & 0.7940 & $\mathrm{~cm}$ \\
\hline Number of large coolant holes & 102 & - \\
\hline Small coolant channel radius & 0.6350 & $\mathrm{~cm}$ \\
\hline Number of small coolant holes & 6 & - \\
\hline Pin pitch & 1.8796 & $\mathrm{~cm}$ \\
\hline Block pitch & 36.0 & $\mathrm{~cm}$ \\
\hline Block (compact) height & 4.928 & $\mathrm{~cm}$ \\
\hline
\end{tabular}


Table 7. Number densities for the fresh fuel block (Exercise I-2a).

\begin{tabular}{|c|c|c|c|}
\hline \multicolumn{2}{|c|}{ Number Densities } & Nuclide & \multirow{2}{*}{$\frac{\mathrm{N}(\mathrm{at} / \mathrm{b}-\mathrm{cm})}{3.67 \mathrm{E}-03}$} \\
\hline \multirow[t]{11}{*}{ TRISO Fuel Particle } & \multirow[t]{4}{*}{ Kernel } & ${ }^{235} \mathrm{U}$ & \\
\hline & & ${ }^{238} \mathrm{U}$ & $1.97 \mathrm{E}-02$ \\
\hline & & ${ }^{16} \mathrm{O}$ & $3.51 \mathrm{E}-02$ \\
\hline & & Graphite & $1.17 \mathrm{E}-02$ \\
\hline & Porous Carbon & Graphite & $5.26 \mathrm{E}-02$ \\
\hline & IPyC & Graphite & $9.53 \mathrm{E}-02$ \\
\hline & \multirow{4}{*}{$\mathrm{SiC}$} & ${ }^{28} \mathrm{Si}$ & $4.43 \mathrm{E}-02$ \\
\hline & & ${ }^{29} \mathrm{Si}$ & $2.17 \mathrm{E}-03$ \\
\hline & & ${ }^{30} \mathrm{Si}$ & $1.39 \mathrm{E}-03$ \\
\hline & & Graphite & $4.79 \mathrm{E}-02$ \\
\hline & OPyC & Graphite & $9.53 \mathrm{E}-02$ \\
\hline \multirow[t]{5}{*}{ Burnable Poison Particle } & \multirow[t]{3}{*}{ Kernel } & B-10 & $2.14 \mathrm{E}-02$ \\
\hline & & B-11 & $8.63 \mathrm{E}-02$ \\
\hline & & Graphite & $2.69 \mathrm{E}-02$ \\
\hline & Buffer & Graphite & $5.02 \mathrm{E}-02$ \\
\hline & $\mathrm{PyC}$ & Graphite & $9.38 \mathrm{E}-02$ \\
\hline \multicolumn{2}{|l|}{ Fuel Compact Matrix } & Graphite & $7.27 \mathrm{E}-02$ \\
\hline \multicolumn{2}{|c|}{ Burnable Poison Compact Matrix } & Graphite & $7.27 \mathrm{E}-02$ \\
\hline \multicolumn{2}{|c|}{ Coolant Channels } & ${ }^{4} \mathrm{He}$ & $2.46 \mathrm{E}-05$ \\
\hline \multicolumn{2}{|l|}{ H-451 Block Graphite } & Graphite & $9.28 \mathrm{E}-02$ \\
\hline
\end{tabular}

Two simplifications have been made to the MHTGR-350 fuel specification. The fuel handling hole and positions for dowels are omitted for simplicity and filled with $\mathrm{H}-451$ block graphite. The $2 \mathrm{~mm}$ gap between fuel blocks is also ignored for these calculations, so the reflective boundary should be applied at the real block boundary. The $\mathrm{UC}_{0.5} \mathrm{O}_{1.5}$ fuel kernels are contained in TRISO fuel particles that have an outer diameter of $855 \mu \mathrm{m}$ and $\mathrm{a}^{235} \mathrm{U}$ enrichment of $15.5 \mathrm{wt} \%$. The packing fraction of the TRISO fuel particles is $35 \%$ in the fuel compacts with the remaining volume being comprised of matrix graphite. Only HFP conditions are considered for these two exercises, as shown in Table 8.

Table 8. HFP operating conditions for Exercise I-2.

\begin{tabular}{|l|c|}
\hline \multicolumn{1}{|c|}{ Parameter/Reactor Condition } & HFP \\
\hline Temperature of all material in fuel compact [K] & 1,200 \\
\hline Temperature of helium in gap [K] & 1,200 \\
\hline Temperature of H-451 block graphite [K] & 1,000 \\
\hline Temperature of all materials in burnable poison compact $[\mathrm{K}]$ & 1,000 \\
\hline
\end{tabular}




\subsection{Exercise I-2c: Super-Cell (Mini-Core) Lattice Calculation}

The use of reflective boundary conditions for fuel blocks next to the inner or outer reflectors lead to significant spectral variances, since these blocks are not surrounded by an infinite lattice of fuel blocks. ${ }^{7}$ To investigate the effect of neighboring blocks on a typical lattice calculation, an example of a super-cell or mini-core has been defined, as presented in Figure 4. Block 26 of the MHTGR-350 is surrounded by reflector blocks on the right and top boundaries, and by one fresh and two depleted fuel blocks on the left and lower boundaries (the block numbering is taken from the OECD/NEA OECD MHTGR-350 benchmark ${ }^{5}$ ).

A simplified super-cell with reflective boundary conditions is shown in Figure 5. One fresh fuel block is positioned in the center of a hexagonal cell. The neighboring blocks on the left side are depleted fuel blocks, and the blocks on the right side are reflector blocks. An important further simplification is the requirement to only model the central fresh fuel block in all its heterogeneous detail (LBP and TRISO compacts), and to lump the depleted fuel and reflector blocks into homogenized regions. This assumption relaxes the significant memory and computational resources required to model this super-cell in full detail. Only the HFP conditions are required to be calculated (Table 10).

To preserve the effort already performed by several participants for Phase III of the OECD MHTGR-350 Benchmark, ${ }^{5}$ the same super-cell specifications will be used for Exercise I-2c. However, the focus is not so much on the absolute results ( $\mathrm{k}_{\infty}$, reaction rates), but on the propagation of the fine-group co-variance uncertainties as input into this calculation. The calculation is performed on a super-cell arrangement with dimensions and number density data identical to those given in Table 6 and Table 7 for the fresh fuel block. The only additional data element is the super-cell flat-to-flat width of $108 \mathrm{~cm}$ $(3 \times 36 \mathrm{~cm})$. The number densities for the reflector region are given in Table 9 .

Table 9. Number densities for the reflector regions.

\begin{tabular}{|c|l|c|}
\hline \multicolumn{1}{|c|}{ Number Densities } & \multicolumn{1}{|c|}{ Nuclide } & $\mathrm{N}(\mathrm{at} / \mathrm{b}-\mathrm{cm})$ \\
\hline \multirow{2}{*}{ H-451 Block Graphite (Reflector Block) ${ }^{\mathrm{c}}$} & Graphite & $9.28 \mathrm{E}-02$ \\
\cline { 2 - 3 } & ${ }^{10} \mathrm{~B}^{\mathrm{d}}$ & $2.76 \mathrm{E}-08$ \\
\hline
\end{tabular}

c In the actual MHTGR-350 design, different graphite grades were used for the reflector and fuel blocks. It is assumed to be identical in this specification, for simplicity.

d The lumped impurities parameters EBC (Equivalent Boron Content) is specified here as ${ }^{10} \mathrm{~B}$. 


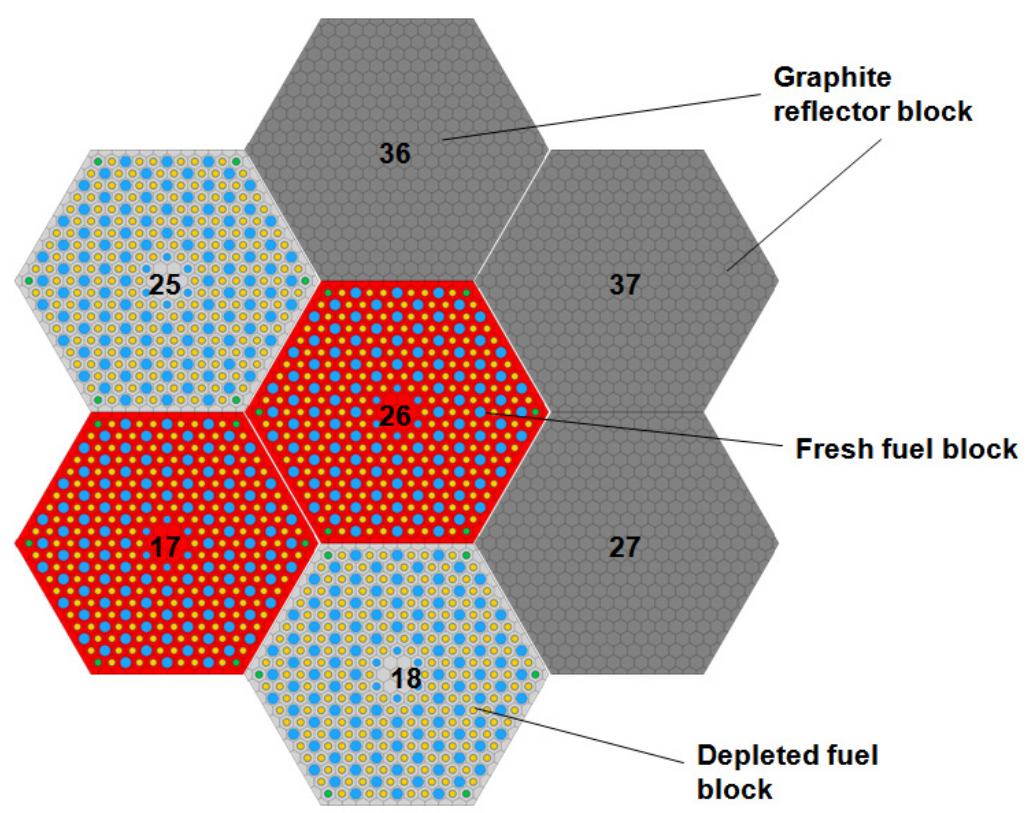

Figure 4. MHTGR-350 super-cell centered at block 26.

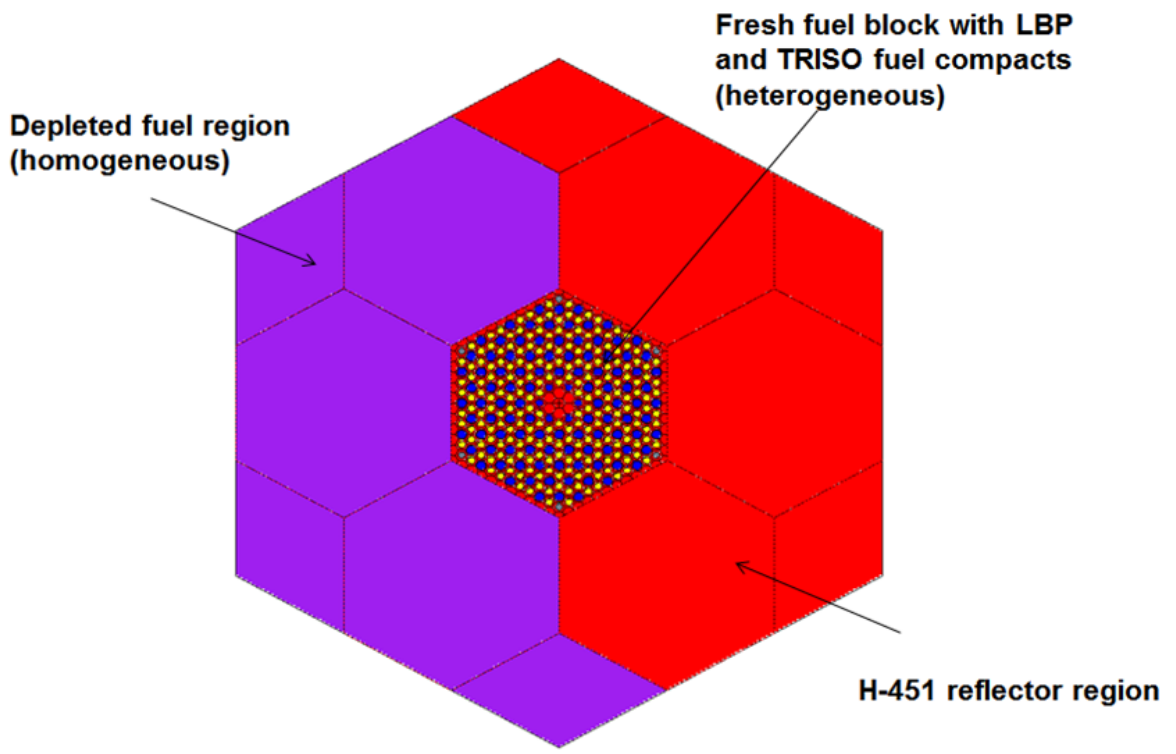

Figure 5. Simplified representation of the Exercise I-2c super-cell.

Table 10. HFP operating conditions for Exercise I-2c.

\begin{tabular}{|l|l|c|}
\hline \multicolumn{1}{|c|}{ Block } & \multicolumn{1}{|c|}{ Parameter / Reactor Condition } & HFP \\
\hline \multirow{4}{*}{ Fresh fuel block } & Temperature of all material in fuel compact [K] & 1,200 \\
\cline { 2 - 3 } & Temperature of helium in gap [K] & 1,200 \\
\cline { 2 - 3 } & Temperature of H-451 block graphite & 1,000 \\
\cline { 2 - 3 } & Temperature of all materials in burnable poison compact [K] & 1,000 \\
\hline \multicolumn{2}{|l|}{ Temperature of homogenized depleted fuel block [K] } & 1,200 \\
\hline \multicolumn{2}{|l}{ Temperature of H-451 graphite reflector block [K] } & 1,000 \\
\hline
\end{tabular}




\section{REFERENCE RESULTS: EXERCISE I-1}

Simulations have been performed with the Monte Carlo codes Serpent ${ }^{8}$ and SCALE/KENO-VI ${ }^{9}$ on a fresh fuel compact unit cell of the MHTRG-350 HTGR, as described in Section 3. In addition to a comparison of the codes and the fuel region treatment, KENO-VI calculations for continuous-energy and multi-group libraries have been performed, and the results obtained are compared with a sub-set of the existing body of literature. In the absence of experimental data, the results of the Serpent calculations are used as the reference for comparison with the KENO-VI calculations, due to Serpent's capability to produce a continuous-energy solution with a random particle distribution in the fuel cell.

\subsection{Codes and Models}

The Serpent Monte Carlo Code is a 3-D continuous-energy neutron transport code, and has been developed at the VTT Technical Research Centre of Finland since 2004. It is capable of simulating various fuel assembly geometries and performing burn-up calculations, and can also be utilized for simulating smaller reactor cores. ${ }^{8}$

To provide a better parallelization of the program and the possibility of performing 3-D burn-up calculations, the Serpent code has been rewritten and the latest version (Serpent 2) is scheduled to be released by the end of 2014. A beta version is currently available for licensed users.

For the calculations reported in this paper, the latest beta version of Serpent 2 (Version 2.1.21) was used. To perform additional calculations using the Evaluated Nuclear Data File (ENDF)-B-VII.1 crosssection data library, the source code of this version was slightly modified by the code developer, J. Leppänen. This modification will be included in the next release of Serpent $2 .{ }^{10}$ Since Serpent 2 provides the capability of performing parallel calculations using a combination of Message Passing Interface (MPI) and Open Multi-Processing (OMP) parallelization, the calculations were performed on the Idaho National Laboratory (INL) High Performance Cluster (HPC), typically utilizing five nodes (a total of 160 processors).

The KENO-VI module of the SCALE 6.1 code package was developed at Oak Ridge National Laboratory (ORNL). It is a high-fidelity three-dimensional Monte Carlo criticality code, and ENDF-B-VII.0 continuous-energy or 238 group cross-section data libraries can be applied. ${ }^{9}$ The ENDF-B-VII.1 library is not yet available for use in SCALE.

In addition to the current SCALE 6.1.2 release, results generated by a beta release of SCALE 6.2 (beta Version 3) have also been included in this study. This version includes new 252- and 56-group libraries optimized for LWR lattice applications. The 252-group structure includes, among others, a more detailed representation of the ${ }^{238} \mathrm{U}$ resonance structure, improved resonance self-shielding and a raise of the thermal cut off. ${ }^{31}$ The relative performance of the 252-group library for this HTGR application is also reported here, and a limited comparison performed with the 56-group structure is reported in Appendix A. It should be noted that ORNL also developed a dedicated HTGR 81-group library in 2012 that produced very similar results compared to the 238 -group library, without the latter's run-time penalty. ${ }^{11}$ Unfortunately, this library is not implemented in the current versions of SCALE, and informal communications with the SCALE development team confirmed that no plans exist to make this library available in external code releases. Some calculations with this library have been performed in the context of this work. For this purpose, SCALE/NEWT was utilized to collapse the 238-group library into the 81-group structure. The results of these calculations are presented in Appendix A. All SCALE calculations were performed on a single processor. Although Version $6.2 \mathrm{~b} 3$ can be compiled to perform parallel KENO-VI computations, efforts to create a parallel version on the INL HPC were not successful.

\subsubsection{Exercisel-1a Serpent and KENO-VI Models}

For Serpent the homogeneous unit cell is modeled as a 2-D cell with reflective boundary conditions in all directions. Cross-section data for the specified temperatures of the CZP and HFP states could directly 
be applied or obtained by Doppler-broadening. In terms of carbon, the natural composition of carbon was applied in all materials. Furthermore, carbon was always considered as graphite (i.e., the thermal scattering data for graphite was applied). Additionally, a Doppler-Broadening Rejection Correction (DBRC) for ${ }^{238} \mathrm{U}$ has been applied. ${ }^{12}$ Several simulations were performed using the ENDF-B-VII.0 and ENDF-B-VII.1 continuous-energy cross-section data libraries.

For each Serpent Monte Carlo simulation, 500 active neutron cycles with 50,000 neutrons per cycle were calculated. The first 50 cycles were skipped and not considered in the evaluation of the multiplication factor. Each of the Serpent runs was typically completed within 5 minutes, using 5 MPI combined with 31 OMP jobs each.

The KENO-VI model is equivalent to the Serpent model, but had to be extended in the axial dimension, for which the compact height $(4.928 \mathrm{~cm})$ was used. Simulations of both CZP and HFP core states using ENDF-B-VII.0 continuous-energy (CE) and multi-group (MG) cross-section libraries were performed. In the case of the MG cross sections, a lattice cell treatment and the 238-group library were applied. An additional calculation was performed using the 252-group library of SCALE 6.2b3. Furthermore, DBRC for ${ }^{238} \mathrm{U}$ and a problem-dependent temperature correction could be applied for the $\mathrm{CE}$ calculations in SCALE6.2b3. With the same settings as for the Serpent calculations, the CE KENO-VI runs with SCALE 6.1.2 typically took about 4 hours to complete on a single processor, and the respective MG calculation took about 2.5 hours. The simulations with SCALE $6.2 \mathrm{~b} 3$ took about 6 and 3.5 hours, respectively.

\subsubsection{Exercise I-1b Serpent and KENO-VI models}

Exercise I-1b requires explicit modeling of the TRISO fuel particles. Therefore, the Serpent 2-D model was extended to a 3-D model with the height of one fuel compact. The reflective boundary condition in all directions was retained.

Serpent provides the option to disperse the particles randomly in a given volume. After entering the particle specifications, the compact dimensions and the packing fraction, Serpent created a file with the positions of 6,416 randomly distributed particles in the compact (Figure 6).

Additionally, a second model with particles in a regular grid was created. To distribute the particles as uniformly as possible, a layer of 128 particles in a square lattice with a pitch of $0.091 \mathrm{~cm}$ was created (Figure 7). All particles are contained within the cylindrical fuel compact region (i.e., no particles were cut by the outer boundary). A stack of 50 particle layers with a height of $0.09856 \mathrm{~cm}$ each made up a fuel compact. A total of 6,400 particles are inserted in the compact in this manner (i.e., 16 particles less than in the random particle model), and the average packing fraction in the compact is therefore slightly decreased from $35 \%$ to $34.91 \%$ ). Due to larger distances between the particles and the outer cylinder boundary, the local packing fraction in one particle lattice cell increased to about $40.1 \%$. With the same settings as in Exercise I-1a, the simulations took about 6 minutes.

The KENO-VI model for Exercise I-1b corresponds with the Serpent model for regularly distributed TRISO particles in the compact, because the standard geometry options in KENO-VI do not include a random particle distribution. The reflective boundary condition was retained, and for the MG calculations, the DOUBLEHET cell data was specified to consider the double heterogeneous structure of the TRISO particles in the fuel compact. ${ }^{13}$ Using the same settings as in Exercise I-1a, the SCALE 6.1.2 CE and MG simulations took about 43 hours and 3 hours, respectively. The SCALE 6.2b3 calculations again took longer with approximately 48 and 4 hours, respectively. 


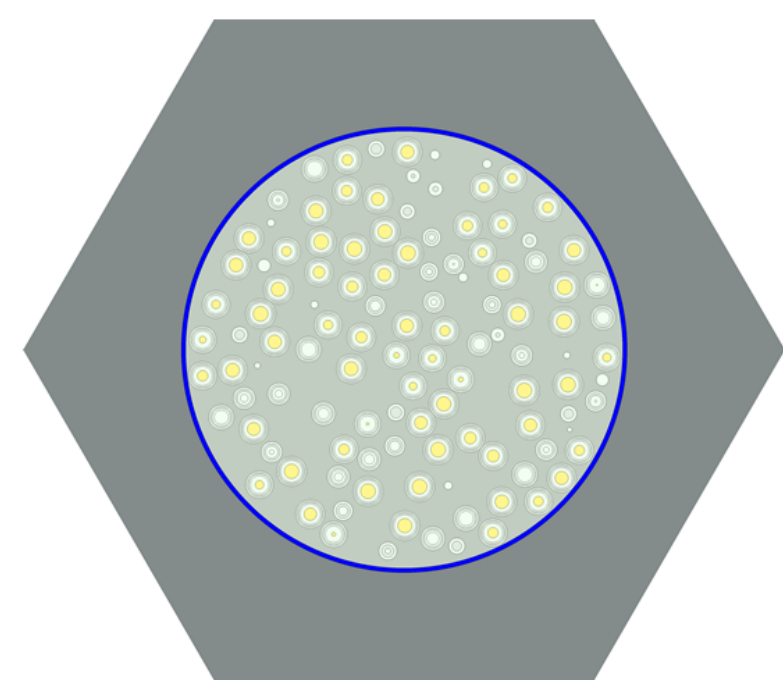

Figure 6. Cross-sectional view of the Serpent model for Exercise I-1b with randomly distributed TRISO particles.

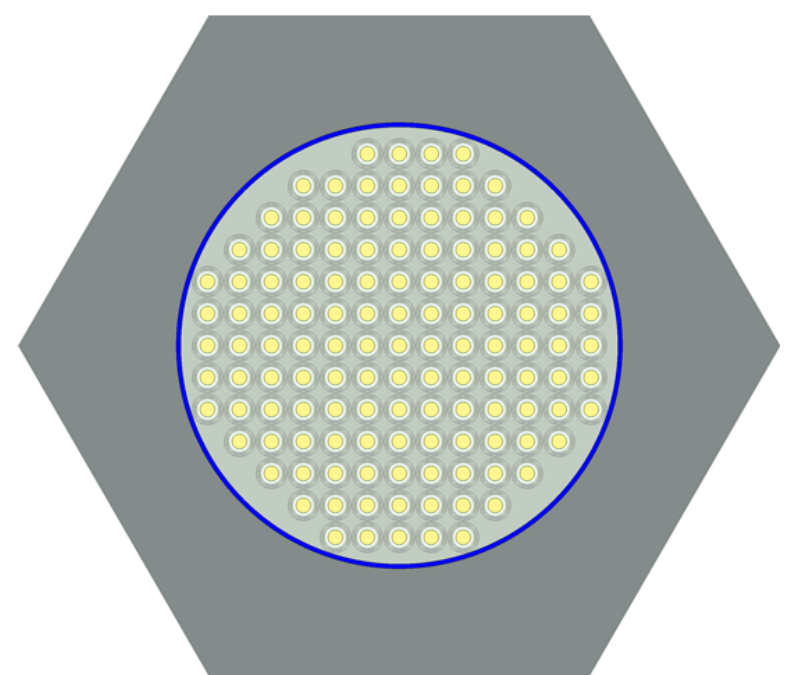

Figure 7. Cross-sectional view of the Serpent model for Exercise I-1b with uniformly distributed TRISO particles.

\subsection{Reference Results for Exercise I-1}

In this section, the reference results obtained for Exercise I-1 are summarized. The term "reference" is used here in the context of the CRP scope where the eventual focus will be on the propagated uncertainties. The reference results correspond to the cases where all input parameters are set to nominal/best estimate values. In the absence of experimental or analytical results for these exercises, the Serpent solutions utilizing random particle distributions are used as the basis for the comparison of all other results.

\subsubsection{Convergence Behavior}

To discard a sufficient number of initial neutron cycles in the Monte Carlo simulations, the convergence of the multiplication factor and the Shannon entropy of the fission source distribution ${ }^{32}$ were investigated.

In Figure 8 and Figure 9 the multiplication factor for Exercise I-1a and $1 \mathrm{~b}$ are shown as a function of the first 100 neutron cycles with 50,000 neutrons each. In Figure 10 the corresponding Shannon entropy is presented. All figures show that both the multiplication factor and the entropy converged before the fiftieth neutron cycle. Therefore, it is sufficient to discard the first 50 cycles. The subsequent 500 active cycles was chosen to obtain a target statistical error of less than $20 \mathrm{pcm}$. 


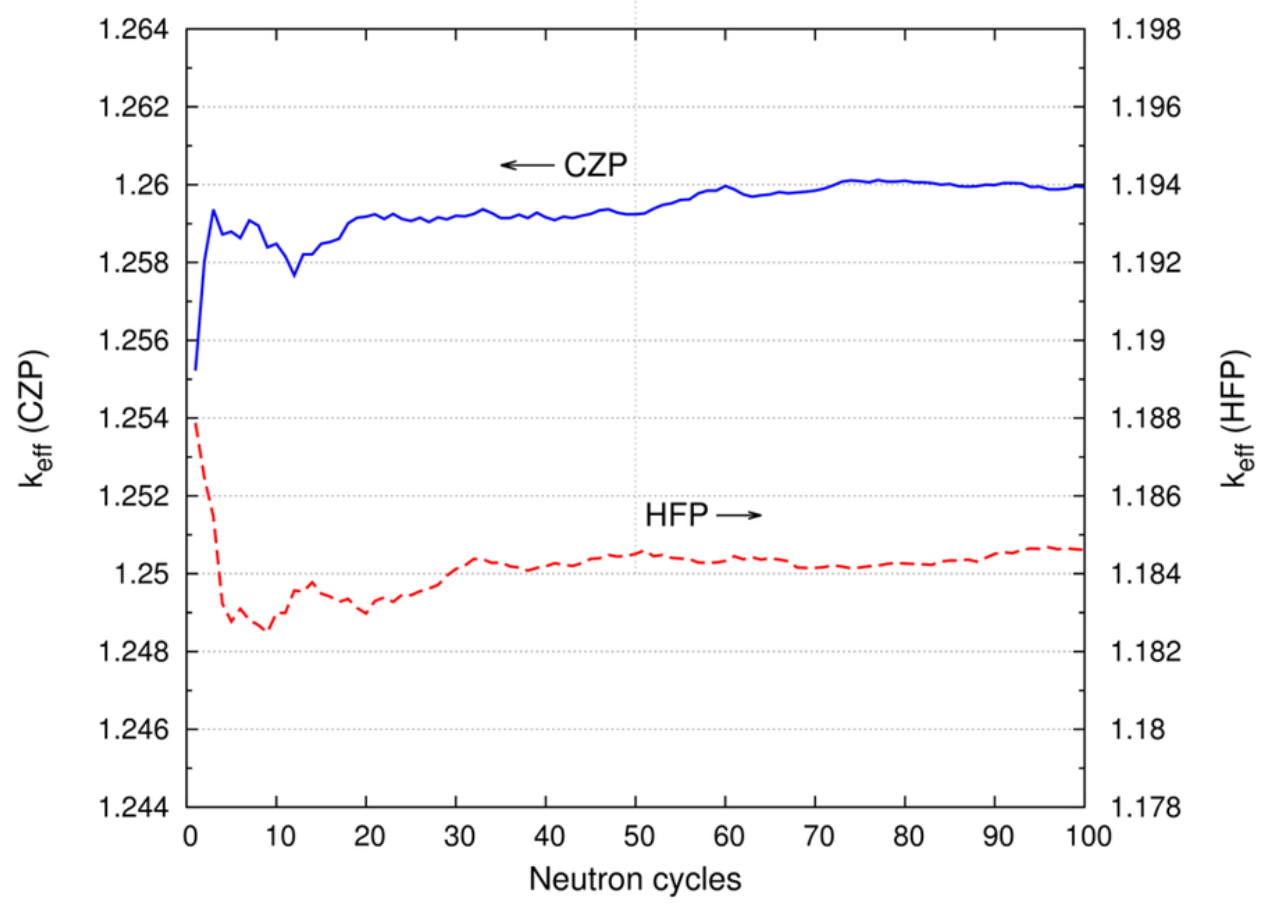

Figure 8. Exercise I-1 a multiplication factor as a function of neutron cycles (50,000 neutrons/cycle).

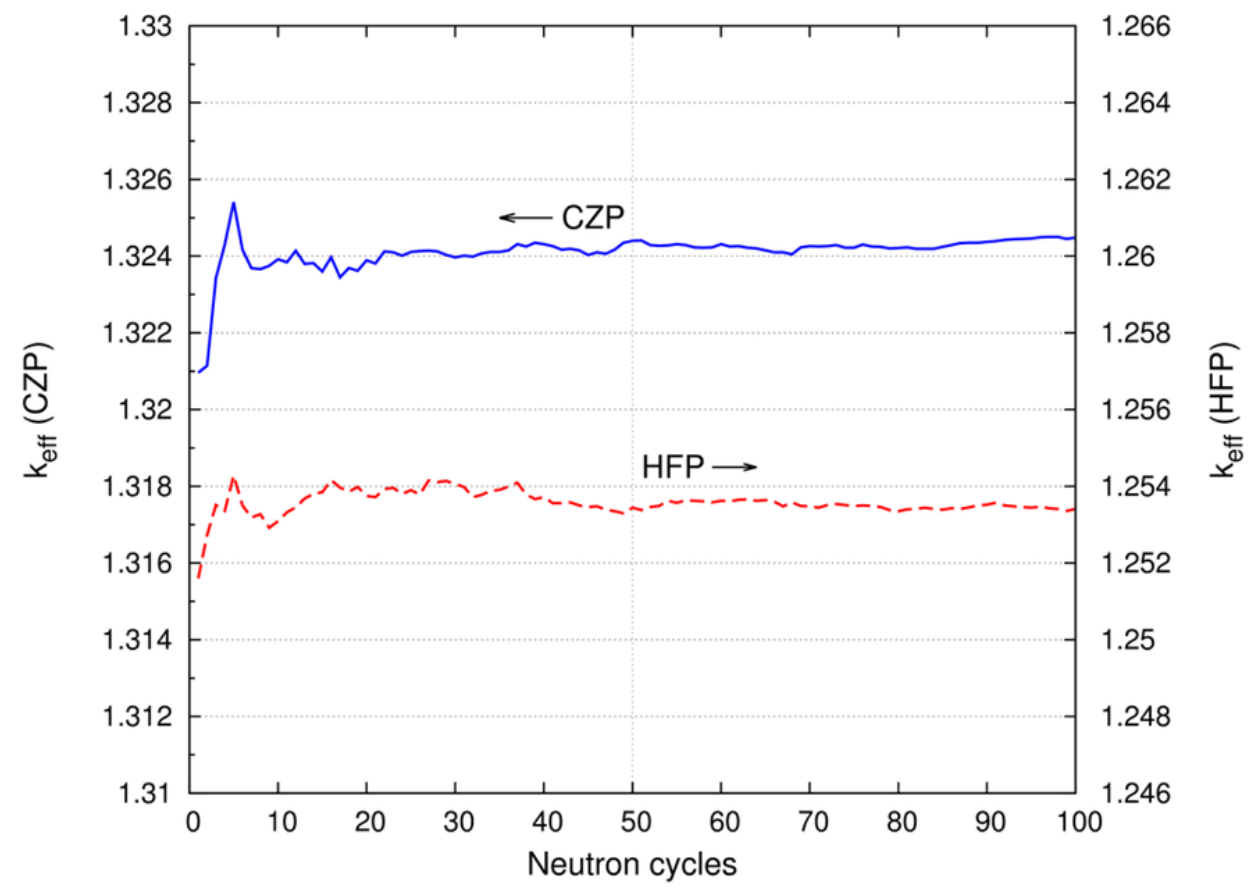

Figure 9. Exercise I-1b multiplication factor as a function of neutron cycles (50,000 neutrons/cycle). 


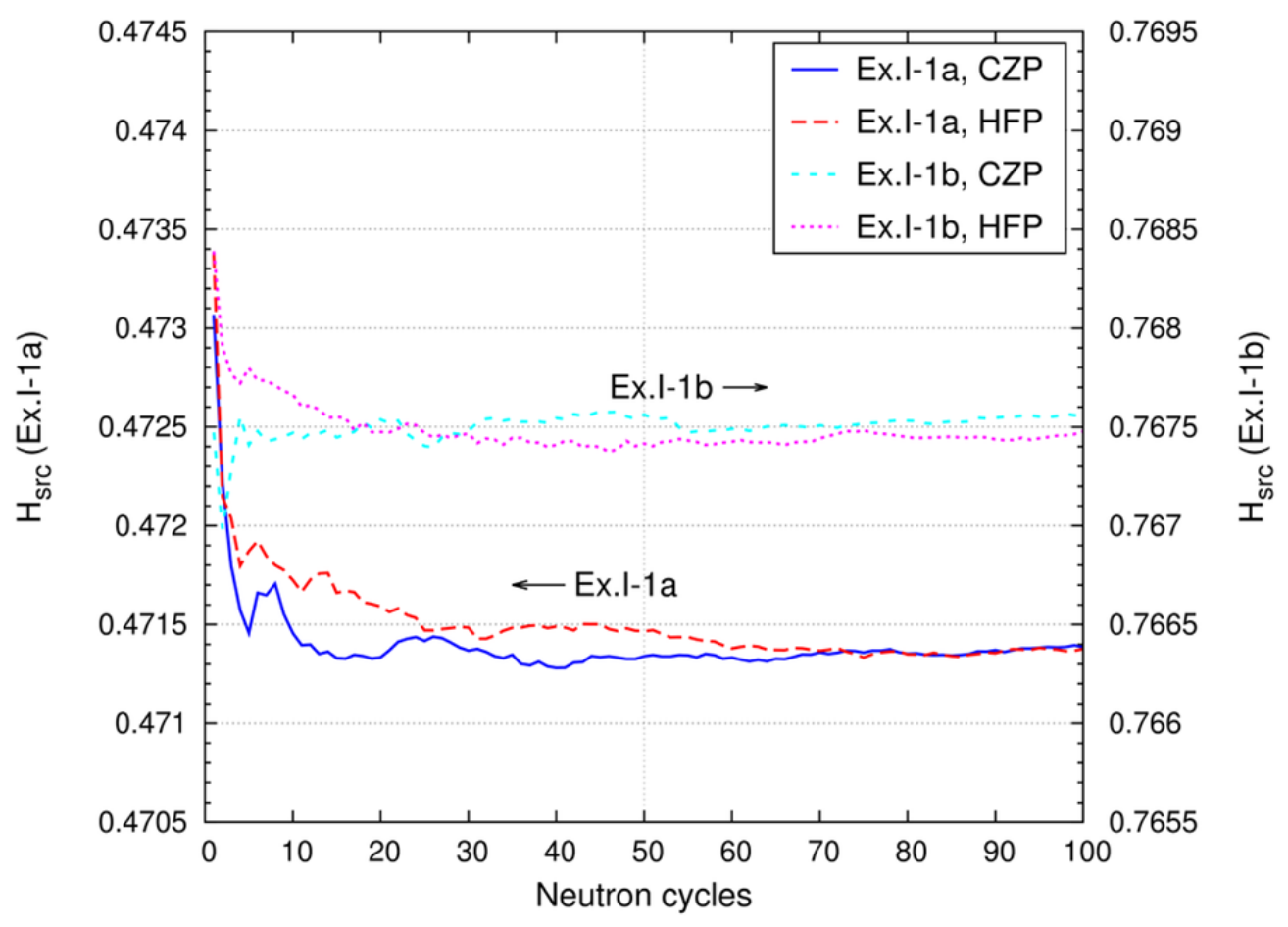

Figure 10. Exercise I-1a/b Shannon entropy of the fission source distribution as a function of neutron cycles (50,000 neutrons/cycle).

\subsubsection{Exercise I-1a}

The results of Exercise I-1a are summarized in Table 11. For this comparison, the result of Serpent using the ENDF-B-VII.0 library is taken as the reference result. It is observed that the use of the ENDF-B-VII.1 data leads to infinite multiplication factors $\left(\mathrm{k}_{\infty}\right)$ more than $100 \mathrm{pcm}$ lower than the ENDF-B-VII.0 values. Goto ${ }^{14}$ ascribed these differences to an underestimation of the carbon neutron capture cross section at thermal energies in the ENDF-B-VII.0 library. The correction of this cross section in ENDF-B-VII.1 resulted in increased neutron capture; thus, a reduction in the multiplication factor.

The HFP $\mathrm{k}_{\infty}$ are furthermore up to 7,500 pcm lower than the CZP $\mathrm{k}_{\infty}$ values. These differences are a primarily the result of ${ }^{238} \mathrm{U}$ Doppler resonance broadening.

Table 11. Multiplication factor for Exercise I-1a.

\begin{tabular}{|l|c|c|c|c|}
\hline \multirow{2}{*}{\multicolumn{1}{c|}{ Model }} & \multicolumn{2}{c|}{ CZP } & \multicolumn{2}{c|}{ HFP } \\
\cline { 2 - 5 } & \multicolumn{2}{c|}{$\begin{array}{c}\Delta \pm \sigma \\
\text { [pcm }]\end{array}$} & $\mathrm{k}_{\infty} \pm \sigma$ & $\begin{array}{c}\Delta \pm \sigma \\
{[\mathrm{pcm}]}\end{array}$ \\
\hline Serpent (ENDF-B-VII.0) & $1.25995 \pm 0.00012$ & (reference) & $1.18462 \pm 0.00014$ & (reference) \\
\hline Serpent (ENDF-B-VII.1) & $1.25841 \pm 0.00013$ & $-153 \pm 18$ & $1.18357 \pm 0.00015$ & $-105 \pm 20$ \\
\hline KENO-VI CE (6.1.2) & $1.26061 \pm 0.00015$ & $66 \pm 20$ & $1.18794 \pm 0.00015$ & $332 \pm 20$ \\
\hline KENO-VI CE (6.2b3) & $1.26080 \pm 0.00014$ & $85 \pm 19$ & $1.18568 \pm 0.00015$ & $106 \pm 20$ \\
\hline KENO-VI 238 MG (6.1.2) & $1.25415 \pm 0.00014$ & $-580 \pm 19$ & $1.18300 \pm 0.00015$ & $-162 \pm 20$ \\
\hline KENO-VI 238 MG (6.2b3) & $1.25420 \pm 0.00015$ & $-575 \pm 20$ & $1.18299 \pm 0.00014$ & $-163 \pm 19$ \\
\hline KENO-VI 252 MG (6.2b3) & $1.25431 \pm 0.00014$ & $-564 \pm 19$ & $1.18435 \pm 0.00014$ & $-27 \pm 19$ \\
\hline
\end{tabular}


The SCALE 6.1.2 CE calculation of the CZP $\mathrm{k}_{\infty}$ is $66 \mathrm{pcm}$ larger than the reference result, and the hot state $\mathrm{k}_{\infty}$ is overestimated by $332 \mathrm{pcm}$. These differences are likely caused by different methods used to generate the ENDF data libraries: Serpent uses the ACE-formatted cross-section libraries of Monte Carlo N-Particle (MCNP), while SCALE utilizes its own processed AMPX format libraries.

The SCALE 6.2b3 CE calculations revealed differences of 85 and $106 \mathrm{pcm}$ to the Serpent reference for the cold and hot state, respectively. The HFP $\mathrm{k}_{\infty}$ difference between SCALE $6.2 \mathrm{~b} 3$ and Serpent is significantly decreased compared to the corresponding SCALE 6.1.2 CE calculation. This improvement can most probably be ascribed to the application of the new DBRC method implemented as a new capability in SCALE $6.2 \mathrm{~b} 3 .^{31}$

A test calculation using SCALE $6.2 \mathrm{~b} 3$ without DBRC for ${ }^{238} \mathrm{U}$ revealed similar differences of more than $300 \mathrm{pcm}$ for both the cold and the hot state compared to SCALE 6.1.2. Application of the DBRC leads to a decrease of the multiplication factor by about $300 \mathrm{pcm}$ in the cold case and by about $570 \mathrm{pcm}$ in the hot case through the inclusion of ${ }^{238} \mathrm{U}$ up-scattering and the resultant increase in resonance absorption. The temperature dependence of this correction is clearly shown by these comparisons. As a result and in contrast to the SCALE 6.1.2 calculations, the differences between the reference Serpent calculations and the SCALE 6.2b3 CE calculations are in the same range for both states.

The 238 group multiplication factors of SCALE 6.1.2 and 6.2b3 have overlapping statistical error bars. The MG calculations of the cold case underestimate $\mathrm{k}_{\infty}$ by more than $550 \mathrm{pcm}$, whereas the hot cases show a decreased deviation of about $160 \mathrm{pcm}$ from the reference value. The SCALE $6.2 \mathrm{~b} 3$ calculation with 252 groups shows overlapping error bars with the other MG calculations for the cold state. However, the multiplication factor of the hot case shows a significant improvement compared to the 238 group calculations, where $\mathrm{k}_{\infty}$ has overlapping error bars with the reference. Since the SCALE 6.2b3 simulations differ only by the applied group structure, these differences are suggested to be a result of the earlier mentioned differences in the libraries.

\subsubsection{Exercise $\mathrm{I}-1 \mathrm{~b}$}

The results of Exercise I-1b are summarized in Table 12. The random particle distribution Serpent model using ENDF-B-VII.0 cross sections is again used as a basis of comparison.

The Serpent calculation with the regular particle lattice exceeds the reference $\mathrm{k}_{\infty}$ value by more than $600 \mathrm{pcm}$. The particle lattice is a simplification of the actual random particle distribution in a compact. Deviations from a random distribution are caused by the lattice arrangement (i.e., the lattice pitch, mass conservation of the fuel [important if particles are cut by the outer compact surface] and the packing fraction). The local packing fraction in the "particle unit cell" is significantly increased compared to the average fraction, which leads to an increase in the multiplication factor.

For a better code-to-code comparison the CE KENO-VI result are also compared to the same Serpent model (i.e., the lattice model). The SCALE 6.1.2 simulation showed differences of 80 and $415 \mathrm{pcm}$ for the cold and hot state, respectively. However, the SCALE 6.2b3 showed overlapping error bars with the Serpent calculation. The significant improvement in the hot case is again suggested to be caused by the application of DBRC, as already noted in the previous exercise.

All MG calculations underestimate the reference by several hundred pcm. The 252 group calculation of the hot state is the only exception, with a smaller difference of $74 \mathrm{pcm}$. For both states, the SCALE 6.2b3 252 MG calculations are the closest to the reference. 
Table 12. Multiplication factor for Exercise I-1b.

\begin{tabular}{|l|c|c|c|c|}
\hline \multirow{2}{*}{\multicolumn{1}{|c|}{ Model }} & \multicolumn{2}{c|}{ CZP } & \multicolumn{2}{c|}{ HFP } \\
\cline { 2 - 5 } & \multicolumn{1}{|c|}{$\mathrm{k}_{\infty} \pm \sigma$} & $\begin{array}{c}\Delta \pm \sigma \\
{[\mathrm{pcm}]}\end{array}$ & $\mathrm{k}_{\infty} \pm \sigma$ & $\begin{array}{c}\Delta \pm \sigma \\
{[\mathrm{pcm}]}\end{array}$ \\
\hline $\begin{array}{l}\text { Serpent - random } \\
\text { (ENDF-B-VII.0) }\end{array}$ & $1.31865 \pm 0.00012$ & (reference) & $1.24657 \pm 0.00013$ & (reference) \\
\hline $\begin{array}{l}\text { Serpent - random } \\
\text { (ENDF-B-VII.1) }\end{array}$ & $1.31767 \pm 0.00012$ & $-98 \pm 17$ & $1.24525 \pm 0.00014$ & $-132 \pm 19$ \\
\hline $\begin{array}{l}\text { Serpent - regular lattice } \\
\text { (ENDF-B-VII.0) }\end{array}$ & $1.32471 \pm 0.00011$ & $606 \pm 17$ & $1.25302 \pm 0.00013$ & $645 \pm 18$ \\
\hline $\begin{array}{l}\text { KENO-VI CE - regular } \\
\text { lattice (6.1.2) }\end{array}$ & $1.32552 \pm 0.00015$ & $687 \pm 20$ & $1.25717 \pm 0.00015$ & $1060 \pm 20$ \\
\hline $\begin{array}{l}\text { KENO-VI CE - regular } \\
\text { lattice (6.2b3) }\end{array}$ & $1.32485 \pm 0.00015$ & $620 \pm 20$ & $1.25300 \pm 0.00018$ & $643 \pm 22$ \\
\hline $\begin{array}{l}\text { KENO-VI 238 } \\
\text { MG/DOUBLEHET (6.1.2) }\end{array}$ & $1.31112 \pm 0.00013$ & $-753 \pm 18$ & $1.24339 \pm 0.00014$ & $-318 \pm 19$ \\
\hline $\begin{array}{l}\text { KENO-VI 238 } \\
\text { MG/DOUBLEHET (6.2b3) }\end{array}$ & $1.31302 \pm 0.00015$ & $-563 \pm 19$ & $1.24493 \pm 0.00013$ & $-164 \pm 18$ \\
\hline $\begin{array}{l}\text { KENO-VI 252 } \\
\text { MG/DOUBLEHET (6.2b3) }\end{array}$ & $1.31308 \pm 0.00015$ & $-556 \pm 19$ & $1.24583 \pm 0.00013$ & $-74 \pm 18$ \\
\hline
\end{tabular}

\subsubsection{Comparison of Exercise I-1a and I-1b}

The main differences between Exercise I-1a and $1 \mathrm{~b}$ are presented in Table 13. The homogenization effect of the fuel compacts leads to an underestimation of the multiplication factor of about $6,000 \mathrm{pcm}$. A similar result was also observed by Kim, ${ }^{15}$ who found differences of about $3,840 \mathrm{pcm}$ at $600 \mathrm{~K}$ and about 4,290 pcm at 1,000 K in SCALE MG and McCARD CE calculations of a single HTGR fuel pin.

Table 13. Comparison between Exercise I-1a and I-1b.

\begin{tabular}{|l|c|c|c|c|}
\hline \multirow{2}{*}{\multicolumn{1}{|c|}{ Model }} & \multicolumn{2}{|c|}{ CZP } & \multicolumn{2}{c|}{ HFP } \\
\cline { 2 - 5 } & \multicolumn{1}{|c|}{$\mathrm{k}_{\infty} \pm \sigma$} & $\begin{array}{c}\Delta \pm \sigma \\
{[\mathrm{pcm}]}\end{array}$ & $\mathrm{k}_{\infty} \pm \sigma$ & $\begin{array}{c}\Delta \pm \sigma \\
{[\mathrm{pcm}]}\end{array}$ \\
\hline $\begin{array}{l}\text { Serpent - random } \\
\text { (ENDF-B-VII.0): het. }\end{array}$ & $1.31865 \pm 0.00012$ & (reference) & $1.24657 \pm 0.00013$ & (reference) \\
\hline $\begin{array}{l}\text { Serpent (ENDF-B-VII.0): } \\
\text { homogeneous }\end{array}$ & $1.25995 \pm 0.00012$ & $-5870 \pm 17$ & $1.18462 \pm 0.00014$ & $-6195 \pm 20$ \\
\hline $\begin{array}{l}\text { KENO-VI CE (6.1.2): } \\
\text { homogeneous }\end{array}$ & $1.26061 \pm 0.00015$ & $-5804 \pm 20$ & $1.18794 \pm 0.00015$ & $-5863 \pm 20$ \\
\hline $\begin{array}{l}\text { KENO-VI 238 MG (6.1.2): } \\
\text { homogeneous }\end{array}$ & $1.25415 \pm 0.00014$ & $-6450 \pm 19$ & $1.18300 \pm 0.00015$ & $-6357 \pm 20$ \\
\hline
\end{tabular}

\footnotetext{
e A calculation using the random particle distribution with 6,400 TRISO particles was also performed to obtain the influence of the slightly smaller amount of fuel in the particle lattice model. The difference between the calculations of 6,400 and 6,416 particles is $112 \mathrm{pcm}$ in the cold case and $69 \mathrm{pcm}$ in the hot case.
} 
The average neutron flux in the Serpent unit cell is presented in Figure 11 and Figure 12 (the fluxes for the random and regular particle distribution are nearly identical, and are not compared here). The spectrum shift of the thermal peak value from the cold to the hot state is clearly visible in this figure. It is shown in Figure 12 that the flux depression in the range of the ${ }^{238} \mathrm{U}$ resonances is larger in the homogeneous fuel compact than in the heterogeneous compact. The corresponding capture reaction rates of ${ }^{238} \mathrm{U}$ are shown in

Figure 13.

Due to fuel lumping, fuel self-shielding leads to a significantly decreased ${ }^{238} \mathrm{U}$ neutron absorption in the heterogeneous compact compared to the homogeneous fuel compact. This effect contributes to the decreased multiplication factor calculated for the homogeneous compact.

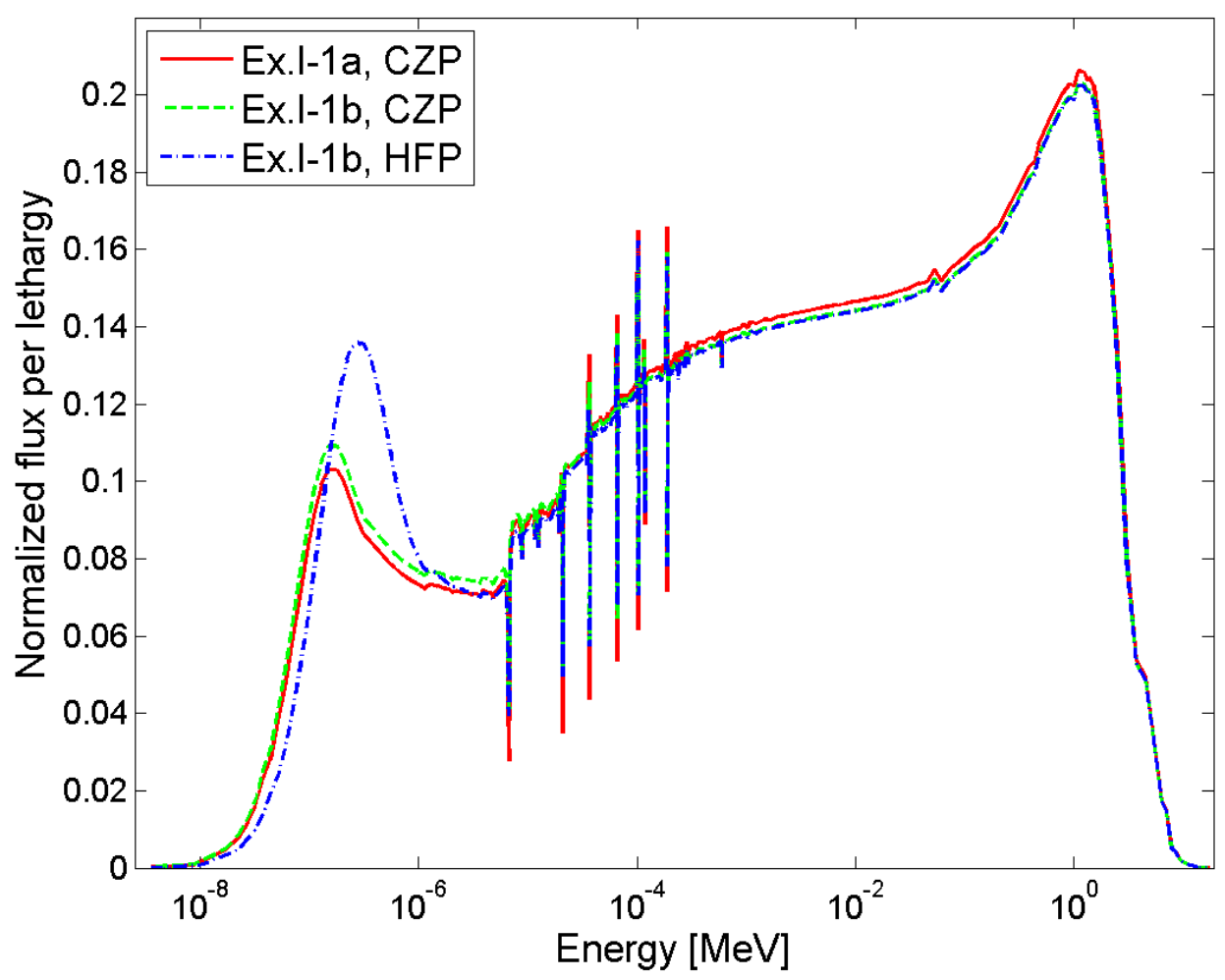

Figure 11. Normalized neutron flux in the fuel compact unit cell (Exercisel-1a and I-1b). 


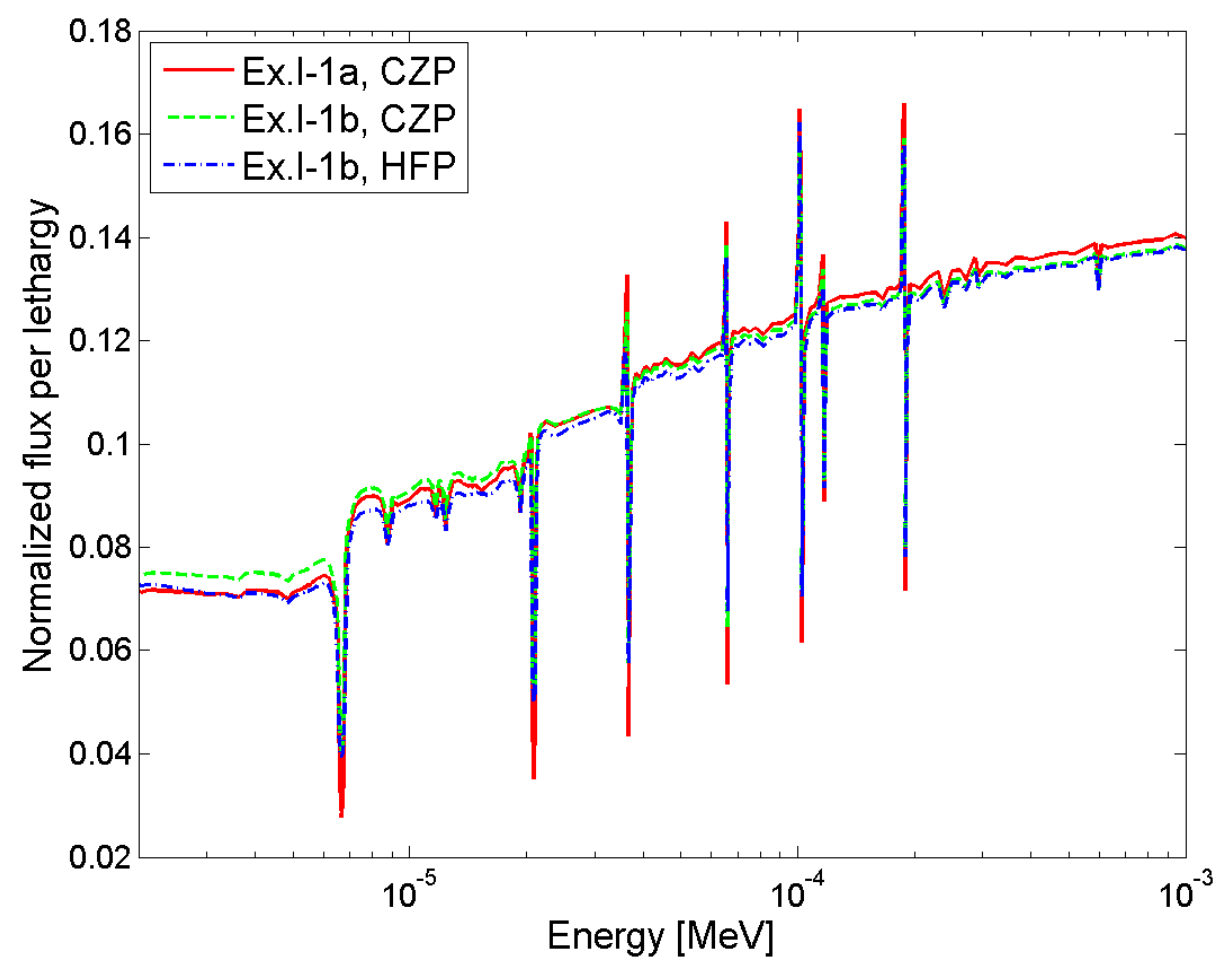

Figure 12. Resonances in the neutron flux of the fuel compact unit cell (extract from Figure 11).

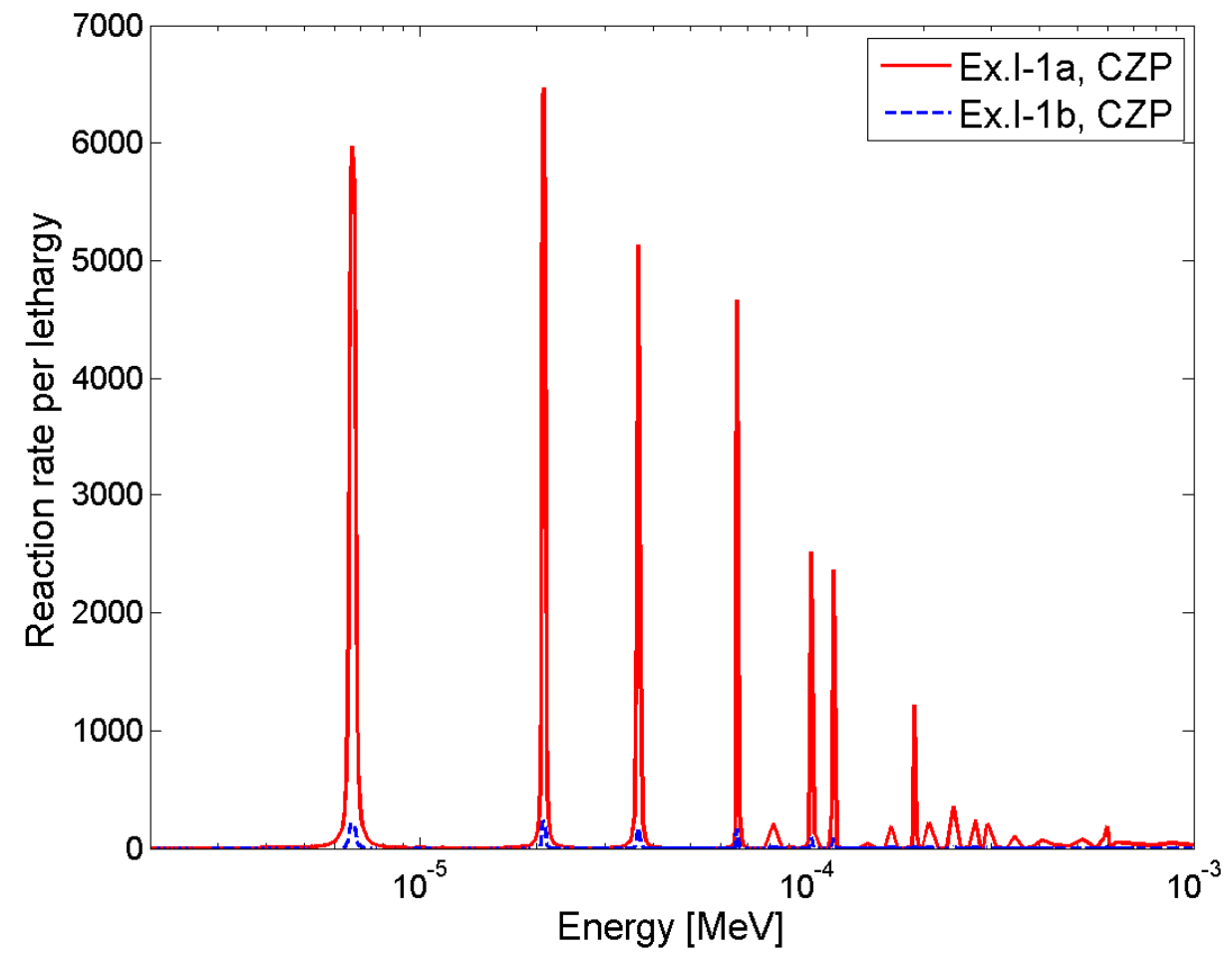

Figure 13. Capture reaction rate of ${ }^{238} \mathrm{U}$ in the CZP state (ExerciseI-1a and I-1b). 
It is interesting that the fast flux peak is higher than the thermal peak for both fuel compact models in Figure 11. It has to be noted that only a unit cell is calculated in this exercise, not a block or a full reactor core. The moderator-to-fuel ratio in this cell is therefore small in comparison with the fuel block, which includes additional graphite in the center, in cells with helium channels and on the outer edges of the block (cf. Section 3.2). If the block graphite density of the cell is increased to match the ratio of a fuel block without burnable poison, similar unit cell and block flux shapes can be obtained (cf. Figure 14). The increased graphite density leads to better moderation and therefore a comparatively higher thermal peak. A similar unit cell spectrum has also been used by Ellis in a very detailed 999 fine-group cell-weighted calculation. ${ }^{11}$

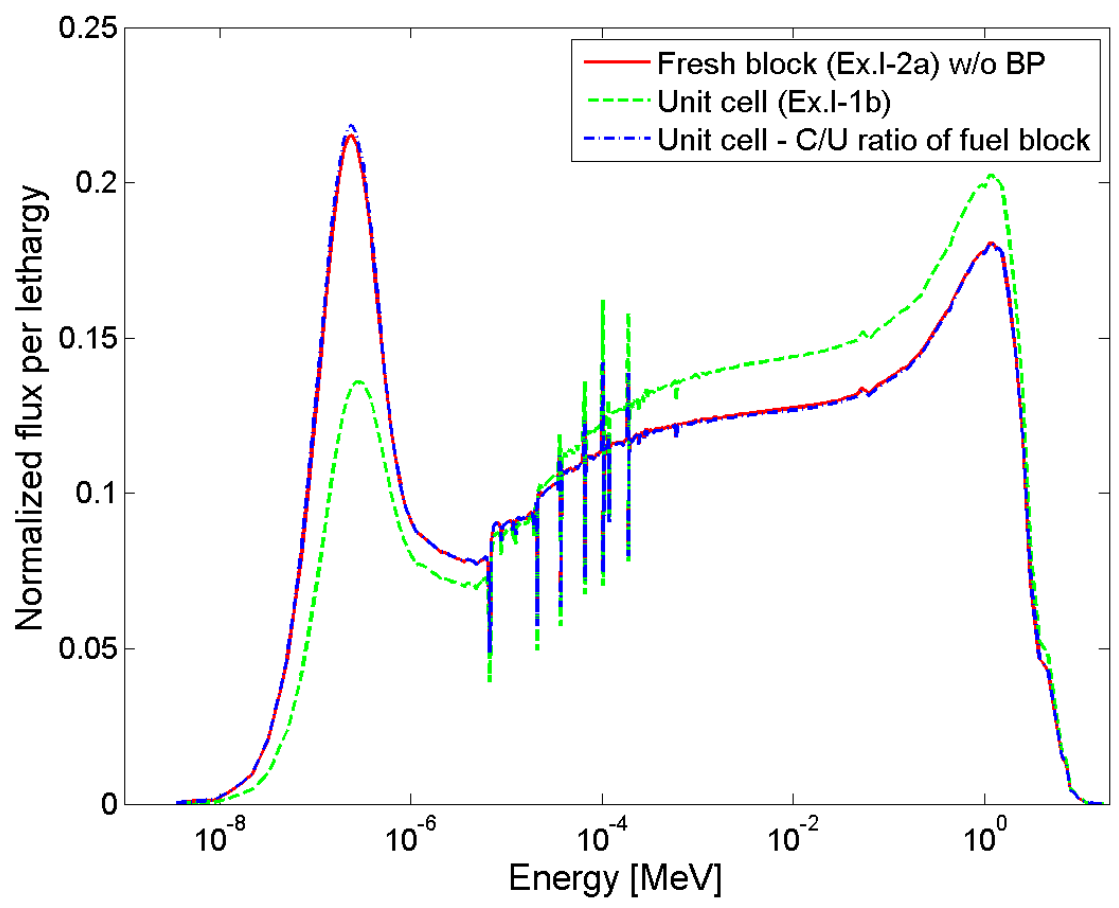

Figure 14. Normalized neutron flux in the unit cell of Exercise I-1b with modified moderator-fuel-ratio.

\subsubsection{Influence of the Graphite Thermal Scattering Library}

During this investigation, the question of the treatment of carbon or graphite in the different materials arose. If the thermal scattering library for graphite is applied, a graphite crystal is assumed. In the case of the UCO fuel particle and the silicon carbide layer, carbon does not form a graphite crystal. The treatment of carbon as graphite in these materials might be wrong. Furthermore, the treatment of other materials containing only graphite is also questionable. Hawari and Gillete ${ }^{17}$ stated that reactor grade graphite should not be assumed to be an ideal single crystal. They considered the porosity of graphite in a calculation of the thermal neutron scattering cross sections for graphite. Swanson and Harrison ${ }^{18}$ suggested modeling graphite structures as a mixture of crystalline-bound graphite and amorphous carbon as a better representation of the actual material.

To estimate the uncertainty that may be introduced by these assumptions, three additional calculations based on Swanson and Harrison's suggestion were performed with Serpent: (1) carbon in all materials was treated as graphite, (2) only the carbon in UCO and the silicon carbide layer was not treated as graphite, and (3) carbon in all materials was treated as a mixture of $80 \%$ graphite and $20 \%$ elemental carbon. In all cases, the models of Exercise I-1b and I-2a were utilized. Simulations were performed with both the random particle distribution and the particle lattice. 
Table 14 and Table 15 provide the results of the simulations with random particle distributions. Case (2) showed minor differences in $\mathrm{k}_{\infty}$ (mostly in the order of one standard deviation and not above $0.05 \%$ ) compared to case (1). The multiplication factor of Case (3) overestimates Case (1) by up to $109 \mathrm{pcm}$ or $0.1 \%$. However, a comparison of these differences did not show a specific bias.

A mixture of graphite and amorphous carbon is not a straightforward modification in the Serpent input. Furthermore, the treatment of carbon in the homogeneous fuel region of Exercise I-1a and in the homogenized burned fuel block of the super cell in ExerciseI-2c would also have to be modified if Case (2) or (3) would be applied. Given the rather small differences between these cases as computed using Serpent, the error introduced by assuming $100 \%$ graphite in all carbonaceous materials is considered negligible for the purposes of this benchmark. Therefore, in all calculations in this study, $100 \%$ graphite is used in all carbonaceous structures.

Table 14. Comparison of three cases of Exercise I-1b (random particle distribution).

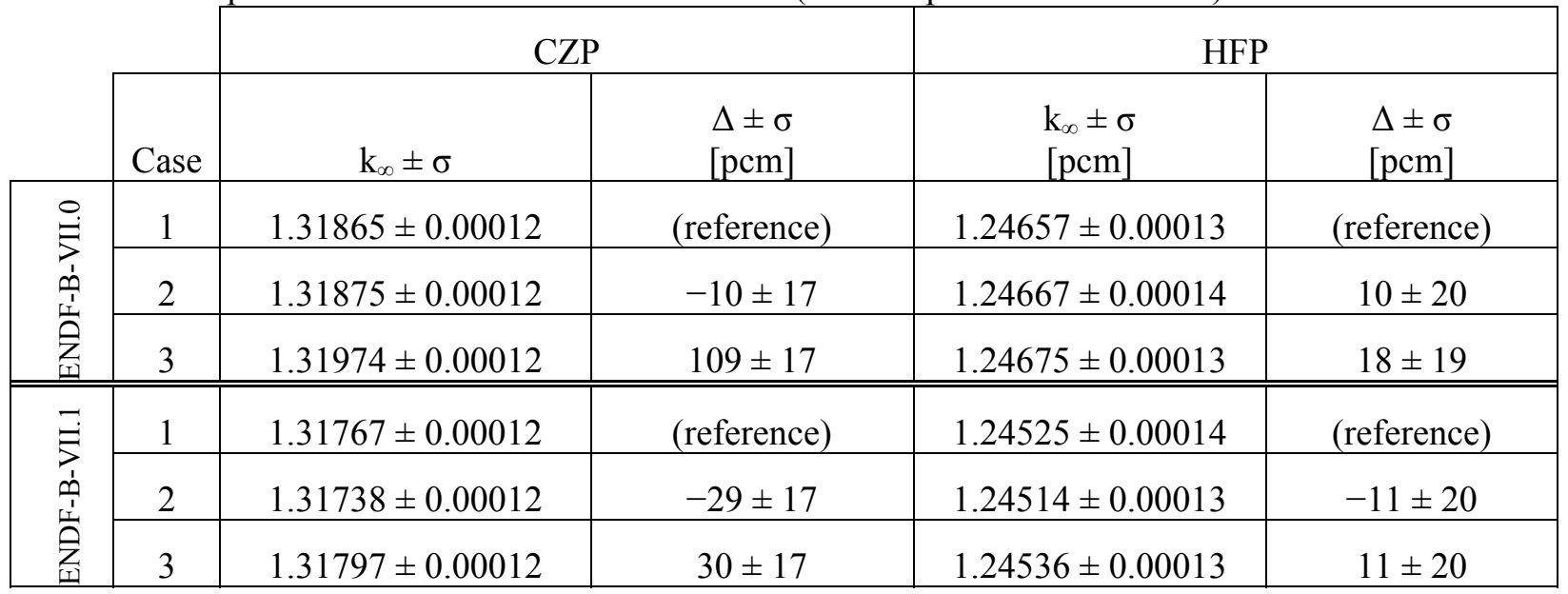

Table 15. Comparison of three cases of Exercise I-2a (random particle distribution).

\begin{tabular}{|c|c|c|c|}
\hline & Case & $\mathrm{k}_{\infty} \pm \sigma$ & $\begin{array}{l}\Delta \pm \sigma \\
{[\mathrm{pcm}]}\end{array}$ \\
\hline \multirow{3}{*}{ 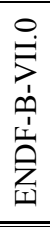 } & 1 & $1.06304 \pm 0.00008$ & (reference) \\
\hline & 2 & $1.06324 \pm 0.00008$ & $20 \pm 12$ \\
\hline & 3 & $1.06386 \pm 0.00008$ & $82 \pm 12$ \\
\hline \multirow{3}{*}{ 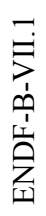 } & 1 & $1.06177 \pm 0.00008$ & (reference) \\
\hline & 2 & $1.06187 \pm 0.00008$ & $10 \pm 12$ \\
\hline & 3 & $1.06278 \pm 0.00008$ & $110 \pm 12$ \\
\hline
\end{tabular}




\section{REFERENCE RESULTS: EXERCISE I-2}

Simulations have been performed with Serpent and SCALE/KENO-VI on a fresh (Exercise I-2a) and a depleted (Exercise I-2b) fuel block of the MHTRG-350 HTGR. Furthermore, a fresh block surrounded by reflector and homogenized burned fuel blocks (a mini-core or super cell) was simulated (Exercise I-2c). The geometries are described in detail in Section 3 (and shown in Figure 2 to Figure 4). In the absence of experimental data, the results of the Serpent calculations including the random particle distributions are again used as the reference for comparison with the KENO-VI calculations.

\subsection{Codes and Models}

\subsubsection{Exercise I-2a Serpent and KENO-VI models}

The fresh fuel block model for Exercise I-2a consists of a lattice of unit cells for each of the components: fuel compact (as used for Exercise I-1b), helium coolant channel, burnable poison (BP), and graphite. Serpent simulations with both random and regular TRISO and BP particle distributions were performed. Reflective boundary conditions were applied in all directions.

For each simulation, 500 active neutron cycles with 200,000 neutrons per cycle were calculated. The first 50 cycles were skipped and not considered in the evaluation of the multiplication factor. Each of the Serpent runs was typically completed in about 1 hour, using five MPI jobs combined with 31 OMP jobs each.

The KENO-VI model for Exercise I-2a is similar to the respective Serpent model for regularly distributed TRISO and BP particles. The reflective boundary condition was retained, and for the MG calculations, the DOUBLEHET cell data was specified to consider the double heterogeneous structure of the TRISO particles in the fuel compact. The BP particles were explicitly modeled in both the $\mathrm{CE}$ and MG calculation, since a DOUBLEHET treatment is not allowed for these particles in SCALE. For each simulation, 500 active neutron cycles with 50,000 neutrons per cycle were calculated and 50 cycles were skipped. The SCALE 6.1.2 CE calculation took about 39 hours, and the MG calculation about 5.5 hours to complete on a single processor. The SCALE $6.2 \mathrm{~b} 3$ calculations were completed in 42 and 6.5 hours, respectively.

\subsubsection{Exercise I-2b Serpent and KENO-VI models}

To obtain the fuel and poison compositions for the burned fuel block, a Serpent burn-up calculation of the fresh fuel block with the random particle distribution and without burnable poison compacts was performed up to $100 \mathrm{MWd} / \mathrm{kg} \mathrm{U}$ at a constant power of $0.1 \mathrm{~kW} / \mathrm{g}$ initial uranium. Because of the symmetry of the block and only small fluctuations of the neutron flux in the fuel region, uniform burn-up in all fuel compacts was assumed. To insure the correct buildup of short-lived fission products (e.g., xenon), small burn-up steps were chosen for the beginning of the burn-up calculation. From a burn-up of $10 \mathrm{MWd} / \mathrm{kg} \mathrm{U}$ onwards, constant steps of $10 \mathrm{MWd} / \mathrm{kg} \mathrm{U}$ were chosen. Constant extrapolation on the predictor and linear interpolation on the corrector was applied.

The Serpent and KENO-VI models of this exercise correspond to the models of Exercise I-2a, with only the fuel compositions replaced by the respective depleted compositions and without the burnable poison compacts. Because of the large number of nuclides that have to be considered in the material definitions, a large amount memory is required for data processing. To make a Serpent simulation possible at all, a lower optimization mode had to be applied, and the time limit of the calculation of one neutron cycle in KENO-VI had to be increased since the standard time limit was exceeded.

With the same settings as in Exercise I-2a, the Serpent calculations took less than 1 hour. The KENO-VI MG calculations took about 5 hours. The CE calculations took 98 and 130 hours for SCALE 6.1.2 and 6.2b3, respectively. 


\subsubsection{Exercise I-2c Serpent and KENO-VI models}

The depleted number densities for the homogenized burned fuel blocks were obtained from the Serpent burn-up calculation performed in Exercise I-2b. To reduce the amount of required memory, the number of nuclides in the homogenized fuel blocks was reduced to oxygen, graphite, hydrogen, isotopes of the main neutron absorbers xenon and samarium, and all available actinides. The super cell model consists of a lattice of one fresh fuel block (Exercise I-2a), the homogenized burned fuel blocks, and graphite reflector blocks. Reflective boundary conditions were applied.

With 500,000 neutrons in 500 active and 50 skipped cycles, the Serpent simulations were completed in less than 3.5 hours. For SCALE the same settings of Exercise I-2a and $2 \mathrm{~b}$ were chosen. The SCALE 6.1.2 CE calculation took about 20 hours and the MG calculation less than 4 hours. The SCALE $6.2 \mathrm{~b} 3$ calculations took a bit longer with 26 and less than 5.5 hours, respectively.

\subsection{Reference Results for Exercise I-2}

\subsubsection{Convergence Behavior}

To discard a sufficient number of initial neutron cycles in the Monte Carlo simulations, the convergence of the multiplication factor and the Shannon entropy of the fission source distribution was investigated. In Figure 15 to Figure 17 the multiplication factor and the Shannon entropy for Exercise I-2 are shown as a function of the first 100 neutron cycles with 50,000 neutrons each. All figures show that both the multiplication factor and the entropy converged before the fiftieth neutron cycle. Therefore, it is sufficient to discard the first 50 cycles. In the Serpent calculations each cycle included 200,000 neutrons. The entropy and $\mathrm{k}_{\infty}$ has converged even earlier in these calculations.

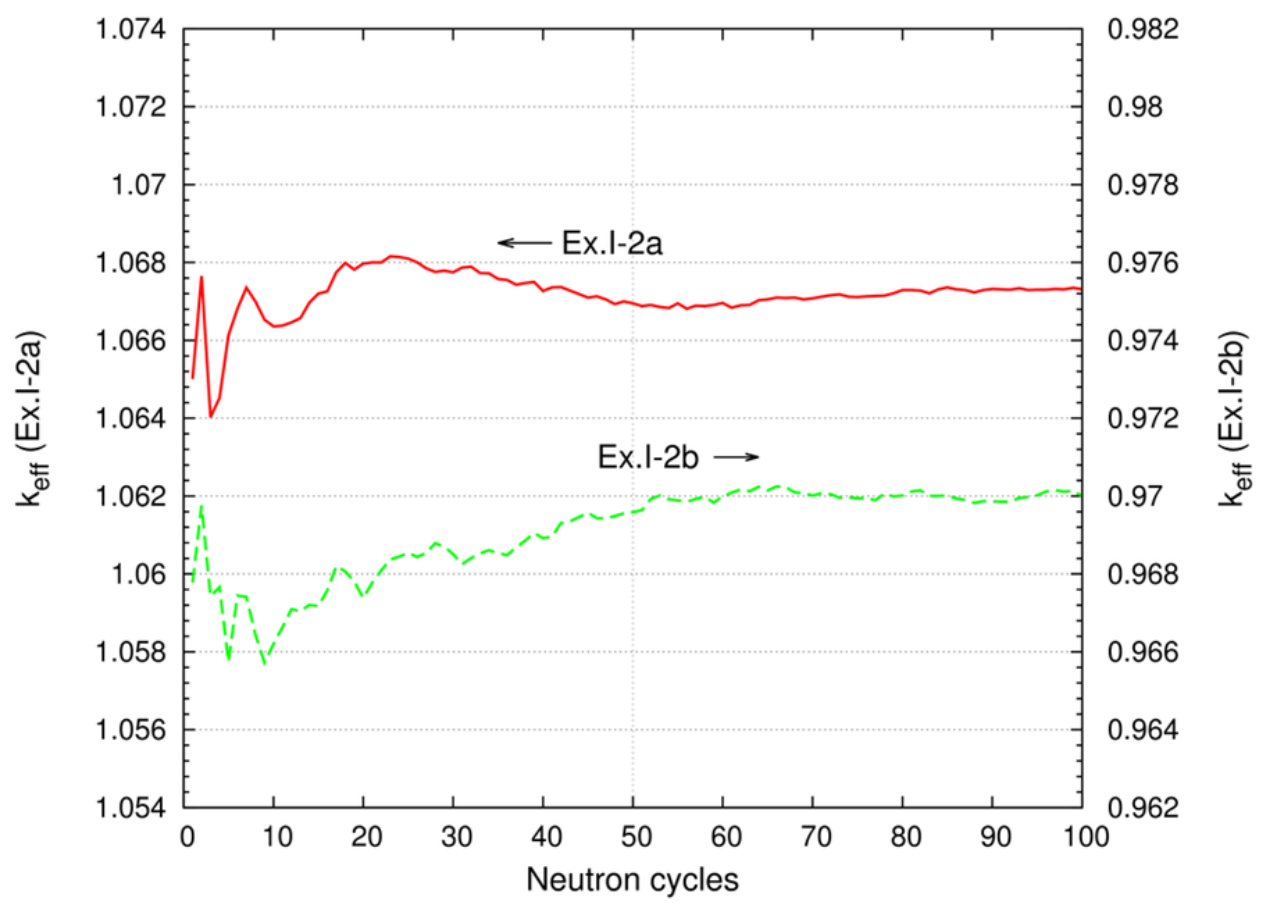

Figure 15. Exercise I-2a/b multiplication factor as a function of neutron cycles (50,000 neutrons/cycle). 


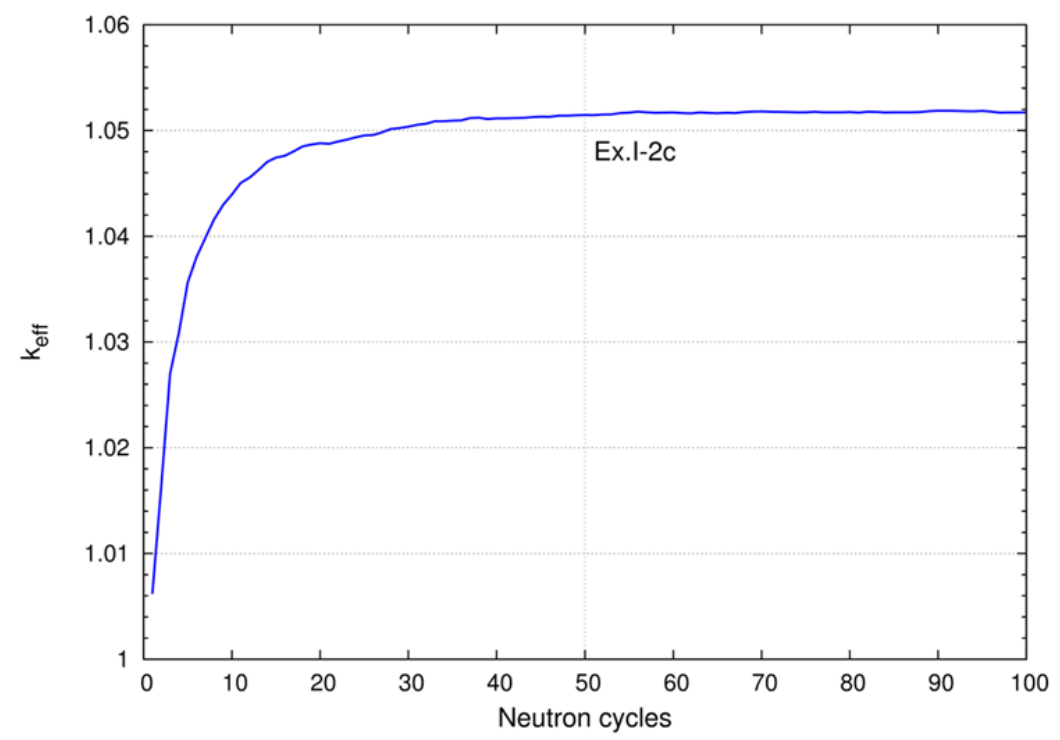

Figure 16. Exercise I-2c multiplication factor as a function of neutron cycles $(50,000$ neutrons/cycle).

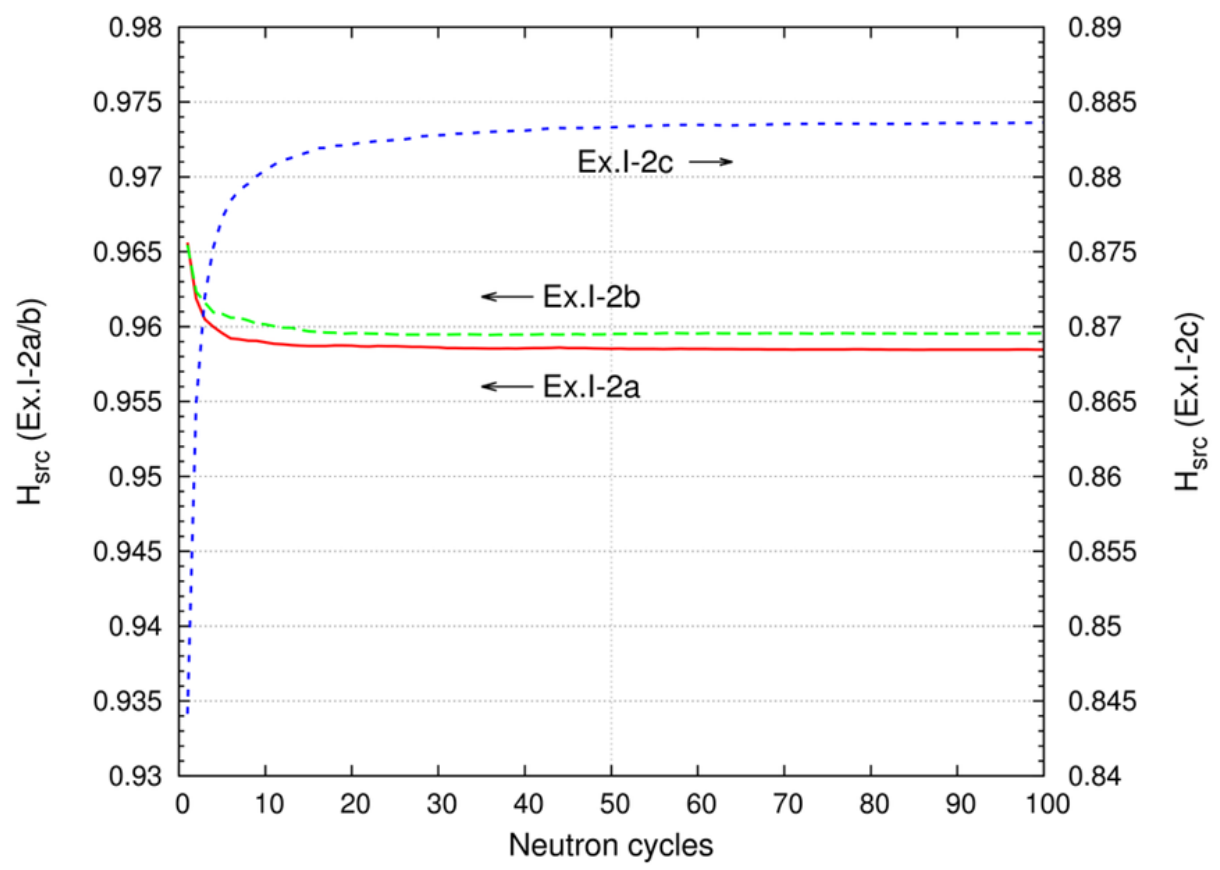

Figure 17. Exercise I-2 Shannon entropy of the fission source distribution as a function of neutron cycles (50,000 neutrons/cycle).

\subsubsection{Exercise I-2a}

The multiplication factors of Exercise I-2a are summarized in Table 16. The Serpent calculation with a random particle distribution and the ENDF-B-VII.0 library is used as the reference result. The use of the ENDF-B-VII.1 library again produced a lower multiplication factor, similar to Exercise I-1b. In comparison to the unit cell calculations, the difference between the random and lattice calculations is decreased. 
The KENO-VI CE kœ values are several hundred pcm higher than the reference for SCALE 6.1.2 and SCALE 6.2b3, respectively. Compared to the Serpent lattice calculation, smaller differences of 472 and $138 \mathrm{pcm}$, respectively, occur. The improvement of the CE calculation in the latest beta version of SCALE 6.2 is similar to the hot unit cell, because the temperature of the fuel block is $1,200 \mathrm{~K}$ in most parts.

All MG calculations overestimate the reference by hundreds of pcm. The calculation with 252 groups obtained the closest match of the DOUBLEHET cases $(328 \mathrm{pcm})$ to the reference value.

Table 16. Multiplication factor for Exercise I-2a.

\begin{tabular}{|l|c|c|}
\hline \multicolumn{1}{|c|}{ Model } & $\mathrm{k}_{\infty} \pm \sigma$ & $\Delta \pm \sigma[\mathrm{pcm}]$ \\
\hline Serpent - random (ENDF-B-VII.0) & $1.06304 \pm 0.00008$ & (reference) \\
\hline Serpent - random (ENDF-B-VII.1) & $1.06177 \pm 0.00008$ & $-127 \pm 12$ \\
\hline Serpent - regular lattice (ENDF-B-VII.0) $\mathrm{f}$ & $1.06766 \pm 0.00008$ & $462 \pm 12$ \\
\hline KENO-VI CE - regular lattice (6.1.2) & $1.07238 \pm 0.00017$ & $934 \pm 19$ \\
\hline KENO-VI CE - regular lattice (6.2b3) & $1.06904 \pm 0.00017$ & $600 \pm 19$ \\
\hline KENO-VI 238 MG/DOUBLEHET (6.1.2) & $1.06945 \pm 0.00014$ & $641 \pm 17$ \\
\hline KENO-VI 238 MG/DOUBLEHET (6.2b3) & $1.07061 \pm 0.00014$ & $757 \pm 17$ \\
\hline KENO-VI 252 MG/DOUBLEHET (6.2b3) & $1.06632 \pm 0.00014$ & $328 \pm 17$ \\
\hline
\end{tabular}

\subsubsection{Burn-up Calculation}

As already mentioned, the fresh fuel block of Exercise I-2a was burned at a constant power density of $0.1 \mathrm{~kW} / \mathrm{g}$ initial uranium up to a final burnup of $100 \mathrm{MWd} / \mathrm{kg} \mathrm{U}$. The development of the multiplication factor over the burn-up period is shown in Figure 18 for both a block with and without burnable poison.

The block with burnable poison reveals a significantly reduced $\mathrm{k} \infty$ in comparison to the block without burnable poison. Both curves show a depression at the beginning of the depletion due to the buildup of the neutron poison xenon. The fuel block without poison shows a continuous decrease of the multiplication factor. It is subcritical at a burnup of $90 \mathrm{MWd} / \mathrm{kg} \mathrm{U}$. however, the block including burnable poison shows a reduced depletion at the beginning of the burn-up as thermal neutrons are absorbed by ${ }^{10} \mathrm{~B}$. With decreasing poison density (see Figure 19) this effect is reduced. This block becomes subcritical at about $40 \mathrm{MWd} / \mathrm{kg} \mathrm{U}$.

The nuclide density of the burnable poison ${ }^{10} \mathrm{~B}$ is decreased from $2.14 \mathrm{E}-02$ (barn $\left.\cdot \mathrm{cm}\right)-1$ in the fresh fuel block to $6.8310-4(\mathrm{barn} \cdot \mathrm{cm})-1$ at a burnup of $100 \mathrm{MWd} / \mathrm{kg}$ U. However, it was expected to deplete to a smaller number density at such a high burn-up. The multiplication factor was further expected to show a notable peak after the initial xenon-depression. The influence of the burnable poison in this depletion calculation was not as large as expected.

\footnotetext{
f A calculation using the random particle distribution (with 6,400 TRISO particles and 46,935 BP particles) was also performed to obtain the influence of the slightly smaller amount of fuel and poison in the particle lattice model. The result of this calculation shows overlapping error bars with the original random calculation.
} 
The reason for this behavior is suggested to be the single block with BP flux spectrum, which is harder than the typical spectrum of a thermal reactor. This shift in the spectrum is mainly the result of the lower moderator-fuel-ratio (as discussed in Section 4.2.3). The flux spectrum of a fresh block with burnable absorbers is compared in Figure 20 to a spectrum of a block without burnable absorbers. Since these flux spectra are not normalized, the influence of the poison is clearly visible. The poison absorbs thermal neutrons; therefore, it significantly decreases the thermal peak. Since the spectrum is comparatively hard, the influence of the poison is comparatively small.

Due to these atypical effects observed, it was decided to define the depletion of a fresh block without burnable poison as the route to obtaining the number densities required for Exercise I-2b.

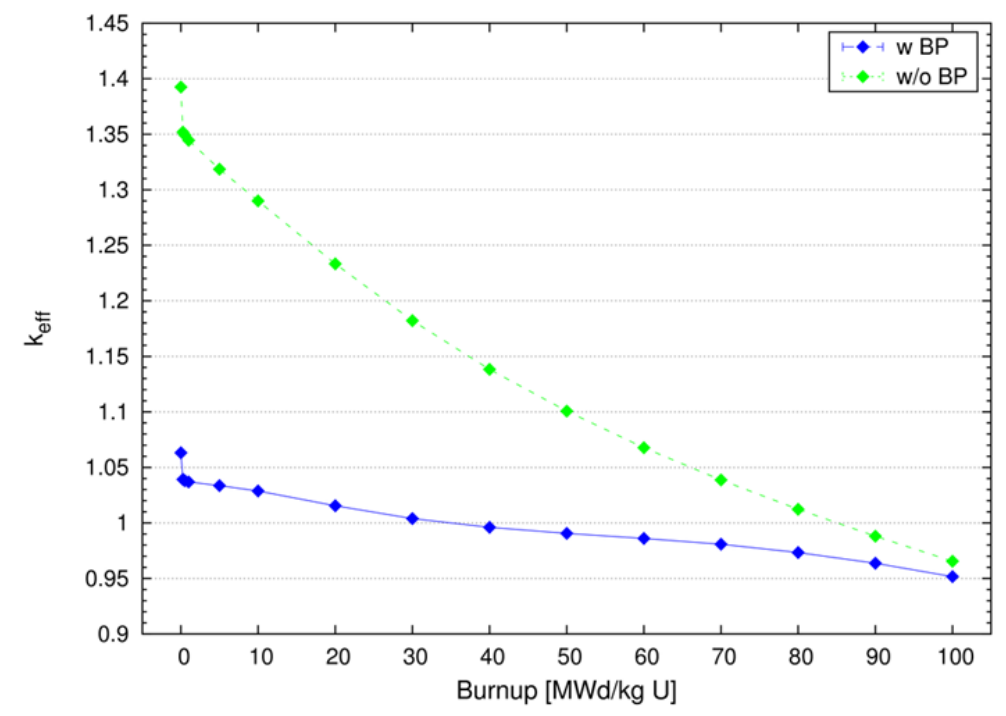

Figure 18. Development of the multiplication factor in a burn-up calculation with and without burnable poison (BP).

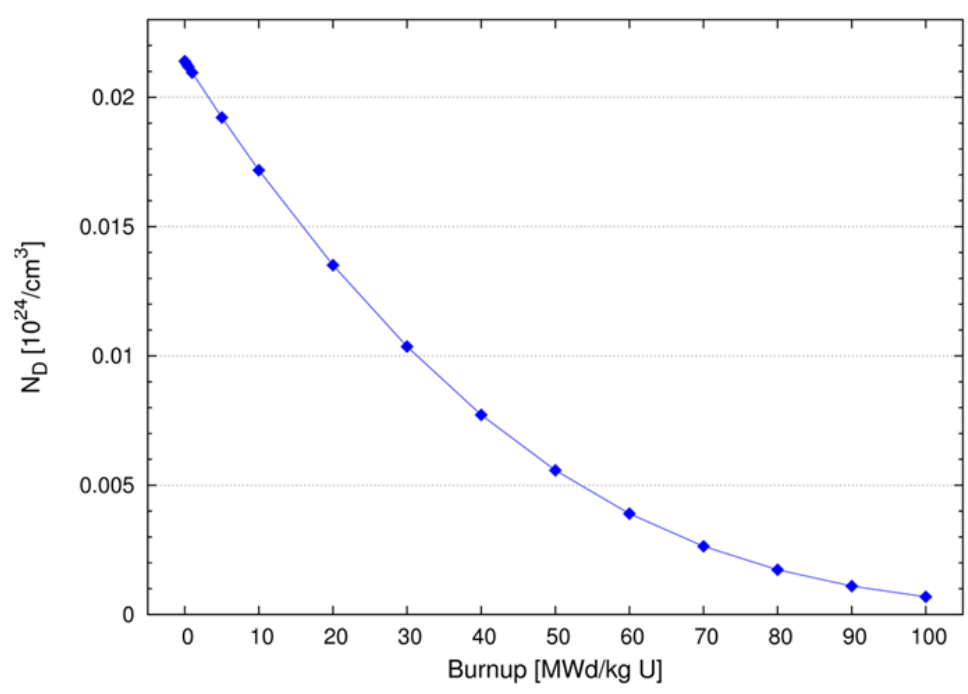

Figure 19. Development of the ${ }^{10} \mathrm{~B}$ number density during the burn-up calculation. 


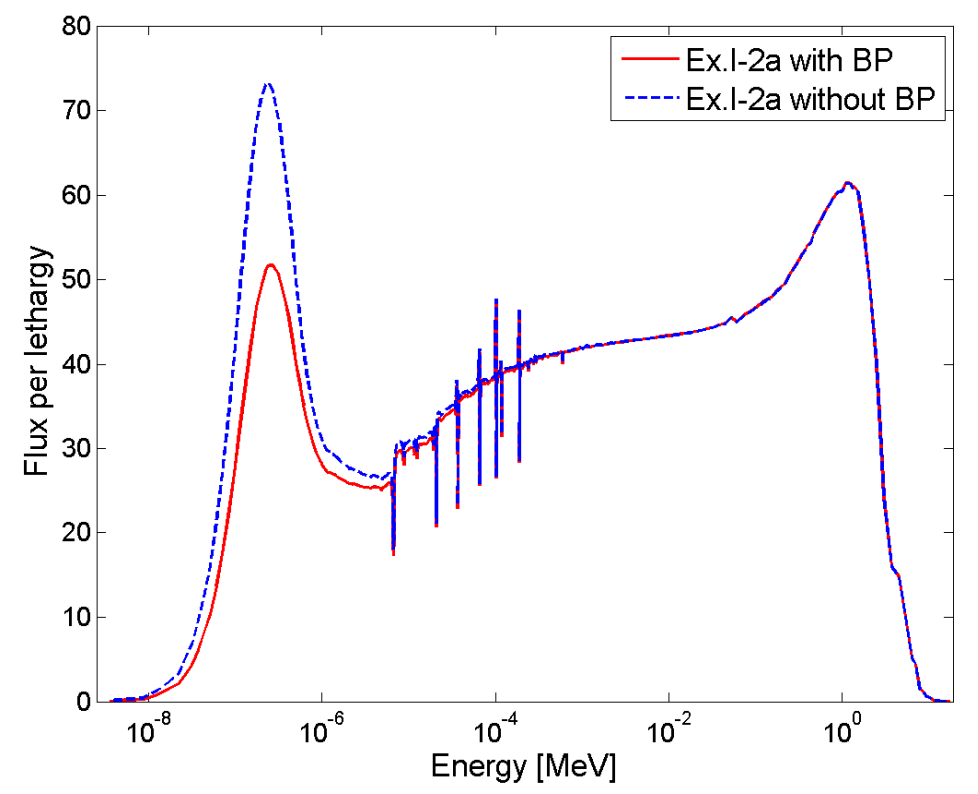

Figure 20. Neutron flux in the fresh fuel block (Exercise I-2a) with and without BP compacts.

\subsubsection{Exercise I-2b}

For the depleted fuel block calculation, it was found that a reduction in the number of nuclides led to a significant reduction in run-time, but that is also led to very large deviations from the reference multiplication factor. In a comparison calculation, only hydrogen, graphite, oxygen, the main neutron absorbers and all available actinides were considered. This calculation was completed in about $40 \%$ of the time that is needed for the $\mathrm{k}_{\infty}$ calculation of a single block using the full set of nuclides available from Serpent. This approach would improve the calculation time of KENO CE models significantly. It did, however, result in a $\mathrm{k}_{\infty}$ overestimation of more than 7,500 pcm, and to differences in the neutron flux up to $5 \%$. Therefore, it was decide to utilize the full set of Serpent isotopes for Exercise I-2b.

It was also observed that at least four significant digits of the number densities are necessary to maintain precision in eigenvalue calculations.

The multiplication factors for the block containing a composition of all available nuclides (a number of 279 nuclides) in the depleted fuel and poison are presented in Table 17. In contrast to all other exercises, the calculation with ENDF.B-VII.1 data revealed a slightly increased multiplication factor. The combined changes in the cross sections of the large number of considered nuclides seem to compensate for the effect of the increased neutron absorption by graphite. The difference between the Serpent random and lattice calculations is similar to the fresh fuel block case.

The SCALE CE calculations show differences to the reference Serpent CE case in the same range than for the fresh block. The difference of the particle lattice calculations between Serpent and SCALE 6.1.2 decreased by a hundred $\mathrm{pcm}$. The MG calculation of SCALE $6.2 \mathrm{~b} 3$ with 252 groups revealed again the closest result to the reference. All MG calculations show smaller differences to the reference Serpent CE case than for the fresh block. 
Table 17. Multiplication factor for Exercise I-2b.

\begin{tabular}{|l|c|c|}
\hline \multicolumn{1}{|c|}{ Model } & $\mathrm{k}_{\infty} \pm \sigma$ & $\Delta \pm \sigma[\mathrm{pcm}]$ \\
\hline Serpent - random (ENDF-B-VII.0) & $0.96528 \pm 0.00013$ & (reference) \\
\hline Serpent - random (ENDF-B-VII.1) & $0.96619 \pm 0.00013$ & $91 \pm 19$ \\
\hline Serpent - regular lattice (ENDF-B-VII.0) & $0.97011 \pm 0.00013$ & $483 \pm 19$ \\
\hline KENO-VI CE - regular lattice (6.1.2) & $0.97381 \pm 0.00018$ & $853 \pm 23$ \\
\hline KENO-VI CE - regular lattice (6.2b3) & $0.97176 \pm 0.00020$ & $648 \pm 24$ \\
\hline KENO-VI 238 MG/DOUBLEHET (6.1.2) & $0.96913 \pm 0.00011$ & $385 \pm 18$ \\
\hline KENO-VI 238 MG/DOUBLEHET (6.2b3) & $0.97025 \pm 0.00011$ & $497 \pm 18$ \\
\hline KENO-VI 252 MG/DOUBLEHET (6.2b3) & $0.96788 \pm 0.00012$ & $260 \pm 18$ \\
\hline
\end{tabular}

\subsubsection{Comparison of Exercise I-2a and Exercise I-2b}

The thermal neutron flux as a function of the location is compared for the fresh and burned fuel block in Figure 21. The x-axis origin is defined in the center and proceeds to the periphery of the fuel block. The depression in the flux profile of the fresh fuel block (red line) between 18 and $20 \mathrm{~cm}$ is related to the burnable poison in the corner of the block. This depression is not visible in the burned block because of the absence of absorber compacts. Small depressions of the thermal flux are also observed at the location of the fuel compacts in both fuel blocks, since thermal neutrons are especially absorbed by the fuel.

A comparison of the normalized flux spectrum for Exercises I-2a and $2 b$ is presented in Figure 22. The thermal peak of the fresh fuel block is significantly decreased because of neutron absorption by the burnable absorbers (cf. Figure 20). The neutron flux spectrum of the burned fuel block shows a smaller thermal peak and additional resonance peaks compared to the fresh block. This is a consequence of the build-up of plutonium in the burned fuel composition.

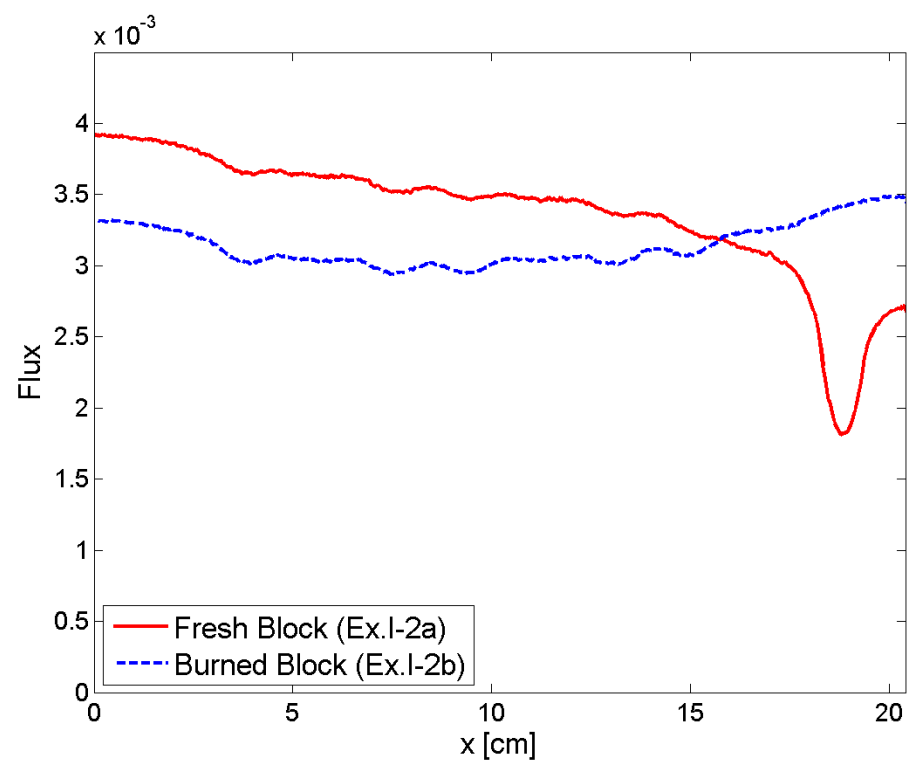

Figure 21. Normalized thermal flux in radial direction (Exercise I-2a and Exercise I-2b). 
The thermo-physical properties of the materials used in Ex. I-3 and I-4 are specified in Table 22 through Table 25.

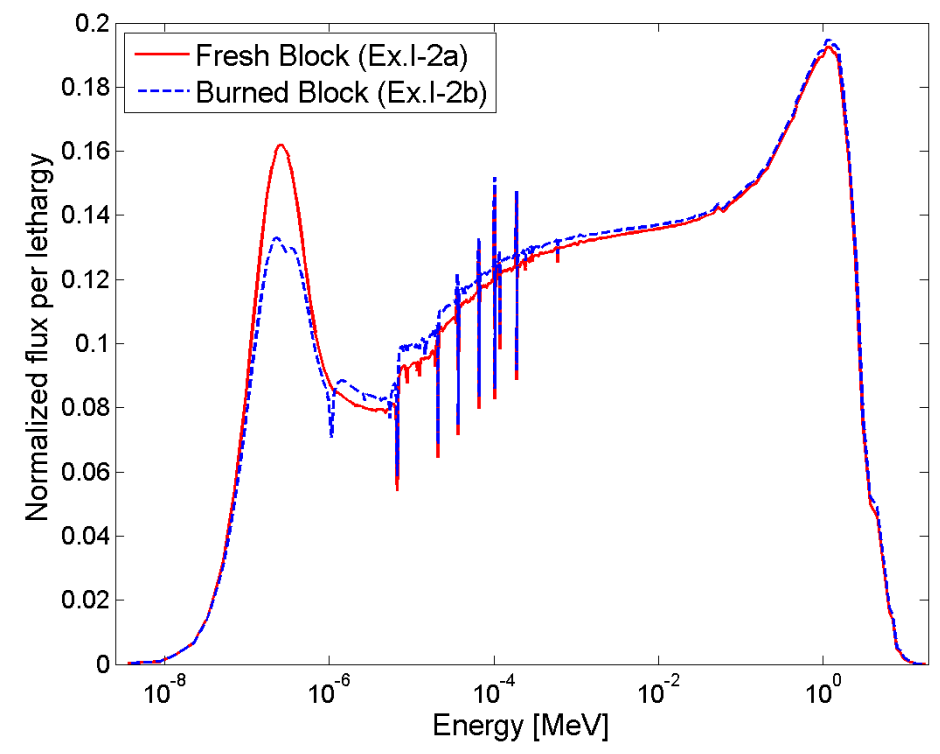

Figure 22. Normalized neutron flux (Exercise I-2a and Exercise I-2b).

\subsubsection{Exercise I-2c}

This super cell considers only a reduced set of 51 nuclides in the homogenized burned fuel region. The effect of this simplification on the multiplication factor is not negligible. If the full set of available nuclides is considered (279) the multiplication factor is $140 \mathrm{pcm}$ larger than in the simplified model. Deviations of up to $30 \%$ in the thermal neutron flux are observed. Since the duration of the calculation is reduced by a factor of more than 2 , and the chosen constitution of the super cell is already a simplification, the simplified composition of the homogenized burned fuel region was nevertheless chosen for investigations of this exercise.

The results obtained by for the super cell are summarized in Table 18 . The difference between the $\mathrm{k}_{\infty}$ Serpent calculations using the random and regular particle distribution is $228 \mathrm{pcm}$, which is smaller than in all previous exercises. The calculation with SCALE 6.1.2 shows a $\mathrm{k}_{\infty}$ within the standard deviation of the Serpent lattice calculation, and SCALE $6.2 \mathrm{~b} 3$ showed a difference of $80 \mathrm{pcm}$. The MG calculations are close to each other within a range of $90 \mathrm{pcm}$; but larger than the reference by more than $200 \mathrm{pcm}$. Problem I-2c produced the best-matched results of all exercises: the KENO results are all within $320 \mathrm{pcm}$ of the reference Serpent result, and the deviations between the various KENO models are within $170 \mathrm{pcm}$.

The neutron spectrum of the super cell is shown in Figure 23. Due to the large amount of reflector graphite around the fuel block and thereby a large moderator-fuel-ratio, the thermal peak is much higher than in the fuel block and the unit cell, and more representative of a typical full-core HTGR spectrum. This is further suggested to be the cause of the large differences (about $670 \mathrm{pcm}$ ) between the Serpent calculations with different ENDF cross-section libraries. 
Table 18. Multiplication factor for Exercise I-2c.

\begin{tabular}{|l|c|c|}
\hline \multicolumn{1}{|c|}{ Model } & $\mathrm{k}_{\infty} \pm \sigma$ & $\Delta \pm \sigma[\mathrm{pcm}]$ \\
\hline Serpent - random (ENDF-B-VII.0) & $1.05010 \pm 0.00005$ & (reference) \\
\hline Serpent - random (ENDF-B-VII.1) & $1.04341 \pm 0.00004$ & $-669 \pm 7$ \\
\hline Serpent - regular lattice (ENDF-B-VII.0) & $1.05238 \pm 0.00004$ & $228 \pm 7$ \\
\hline KENO-VI CE - regular lattice (6.1.2) & $1.05236 \pm 0.00017$ & $226 \pm 18$ \\
\hline KENO-VI CE - regular lattice (6.2b3) & $1.05158 \pm 0.00016$ & $148 \pm 17$ \\
\hline KENO-VI 238 MG/DOUBLEHET (6.1.2) & $1.05292 \pm 0.00013$ & $282 \pm 14$ \\
\hline KENO-VI 238 MG/DOUBLEHET (6.2b3) & $1.05329 \pm 0.00013$ & $319 \pm 14$ \\
\hline KENO-VI 252 MG/DOUBLEHET (6.2b3) & $1.05239 \pm 0.00015$ & $229 \pm 16$ \\
\hline
\end{tabular}

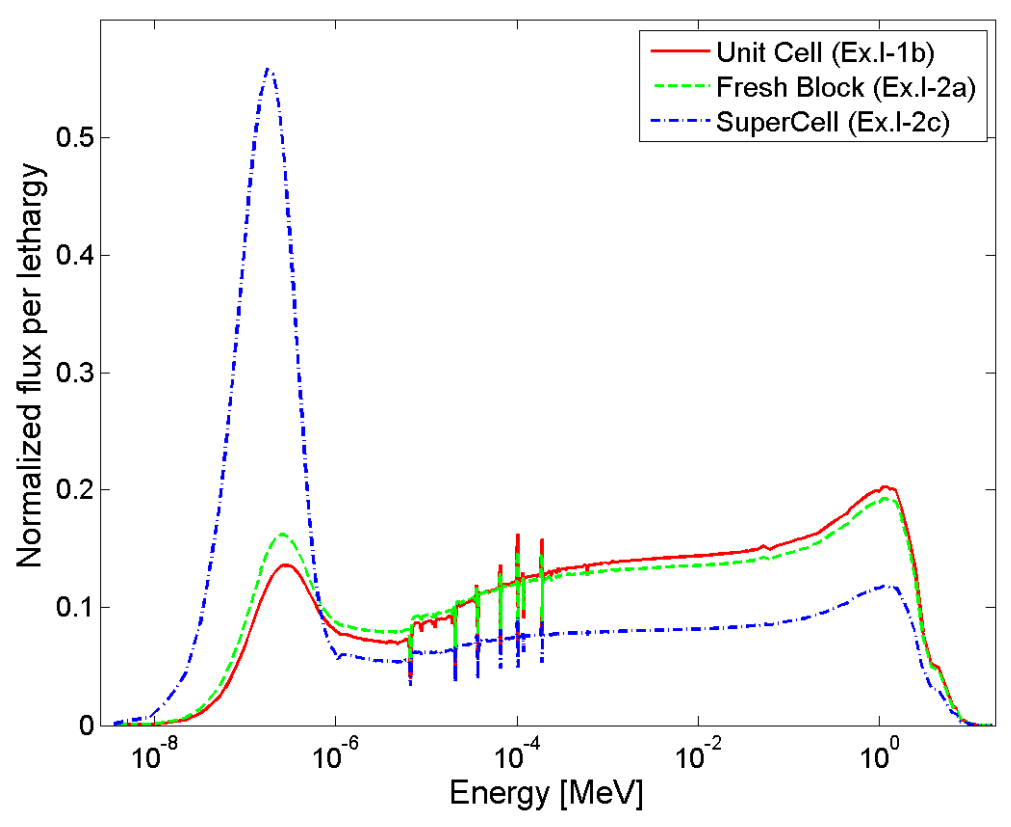

Figure 23. Normalized neutron flux (Exercise I-1b and Exercise I-2a/c).

\subsection{Concluding Remarks: Exercise I-1 and Exercise I-2}

The homogenization of a fuel compact resulted in a significant underestimation of the multiplication factor on the order of several hundred $\mathrm{pcm}$. The general development of the neutron flux is similar to the explicit model. However, large differences in the resonance region were observed due to decreased resonance self-shielding.

$\mathrm{Kim}^{33}$ suggested an adaption of the cylinder radius of the homogeneous fuel compact in order to obtain matching multiplication factors. This reactivity-equivalent physical transformation (RPT) method for homogenization of double-heterogeneous fuel was tested for the MHTGR unit cell. The results of this additional study are reported in Appendix A, where it is shown that the method could be of interest to participants who do not have access to an accurate treatment of the heterogeneous effects. 
Although the results of these MHTGR-350 simulations indicate a trend of overestimating $\mathrm{k}_{\infty}$ in calculations with a regular particle lattice compared to a random particle distribution, comparable studies for prismatic HTGR lattice problems ${ }^{19,20}$ reported conflicting trends, and no consistent bias can be concluded. It is suggested that the trend is dependent on the particle lattice definition. The particle lattice in this study, for example, considered the average particle fraction only in the compact, but not in a particle lattice unit cell.

Except for the super cell, the $\mathrm{k}_{\infty}$ results for all double heterogeneous SCALE 6.1.2 CE calculations exceed the respective Serpent lattice calculations. This trend is consistent with Leppänen's results obtained for a fuel block containing burnable absorber ${ }^{19}$ and with criticality calculations reported by Wang, ${ }^{16}$ Chiang, ${ }^{21}$ and Ilas ${ }^{22}$ for the High Temperature Engineering Test Reactor (HTTR), a prismatic high temperature reactor with annular fuel compacts.

However, the fuel compact simulations of Leppänen ${ }^{19}$ showed well-matched simulations of the regular particle lattice with KENO-VI and Serpent (with overlapping error bars), whereas our results differ by larger margins. As the temperature might have a significant influence on this difference (see especially Exercise I-1b), and Leppänen did not give adequate temperature information, the differences between the two studies cannot be explained at this stage.

Within the set of the SCALE results, an underestimation of $\mathrm{k}_{\infty}$ is observed in all KENO-VI MG calculations compared to the KENO-VI CE results, except for the super cell case. Furthermore, the difference between the KENO-VI MG and CE calculations decreases with an increase in the model size from a unit cell (Exercise I-1b) to a fuel block or a super cell (Exercise I-2). These results agree with the findings of Leppänen for the compact and the prismatic block with burnable absorber. ${ }^{19}$

The super cell showed the smallest difference between the multiplication factors of the KENO-VI CE and MG calculations, and between the random and regular particle distribution in Serpent. Furthermore, it showed consistent results of both the Serpent lattice and the SCALE 6.1.2 CE calculations. As a result of this behavior, the simulations of the MHGTR problems in this work suggest an increasing agreement between the Monte Carlo codes with increasing model size. It also seems appropriate to suggest that participants consider using one or more super cell lattice libraries as input for the Phase II few-group core calculations, since these spectra seems to better represent an HTGR core environment than the single block spectra.

The Serpent calculations using ENDF-B-VII.1 cross sections revealed lower multiplication factors than the respective simulations using ENDF-B-VII.0 data. This has already been attributed to the modified neutron capture cross section in graphite. ${ }^{14}$ These significant differences are far outside one standard deviation and should be studied in detail. However, since a comparison with experimental data is not available for the MHTGR-350, it is not clear which simulation better represents reality. For this aspect, the next section discusses the simulation results of the VHTRC experimental facility.

The results of simulations using SCALE 6.2b3 differ from those of the respective SCALE 6.1.2 simulations. All CE calculations with SCALE 6.2b3 showed smaller multiplication factors than SCALE 6.1.2. An improvement in the new beta version especially for the new 252-group library of SCALE $6.2 \mathrm{~b} 3$ could be observed. However, it should be kept in mind that the SCALE 238 and 252 multi-group structures are optimized for LWR applications, and Version $6.2 \mathrm{~b} 3$ might still be modified before the first production release of SCALE 6.2 by mid-2015. 


\section{EXPERIMENTAL VALIDATION RESULTS: THE VHTRC FACILITY}

There is a significant lack of validation data for prismatic HTGR designs, especially in data sets where information of experimental and material uncertainties are included. For the validation exercise of Phase I, the cell and lattice phases (Exercise I-1 and I-2) are represented by the VHTRC. ${ }^{23}$ The larger HTTR experiment ${ }^{16}$ does not include as much uncertainty information, and is also much more complex to model. This experiment was designed and executed in 1985 at the Japan Atomic Energy Agency (JAEA) Tokai Research and Development Center to verify the calculation accuracy related to the design regarding the neutron physics of the HTTR. Whereas the HTTR experiments included several complex design and operational features, the VHTRC facility was specifically designed as a criticality validation benchmark for the HTTR, which was well-instrumented. An effort was also made to record and quantify experimental uncertainties.

\subsection{VHTRC Description}

A full description is provided in the International Handbook of Reactor Physics Experiments Benchmarks, ${ }^{23}$ but a condensed version is included here for completeness. The VHTRC is a graphite-moderated thermal critical assembly that has a core loaded with pin-in-block fuel of low enriched uranium and a graphite reflector (Figure 24). The assembly has a hexagonal prism shape $(2.4 \mathrm{~m}$ across the flats and $2.4 \mathrm{~m}$ long). In radial direction, the assembly is covered with 0.5 -mm-thick Cd sheets as a thermal neutron absorber, steel frames that have an effective thickness of about $2 \mathrm{~cm}$, and 15 -cm-thick alumina-silica fiber blankets as heat insulation. In axial direction, it is covered with a 0.5 -mm-thick Cd sheet, a steel frame that with an effective thickness of about $0.4 \mathrm{~cm}$ and a 10 -cm-thick alumina-silica fiber blanket. It can be heated up to $200^{\circ} \mathrm{C}$ using 40 electric heaters to study the temperature effect on core characteristics.

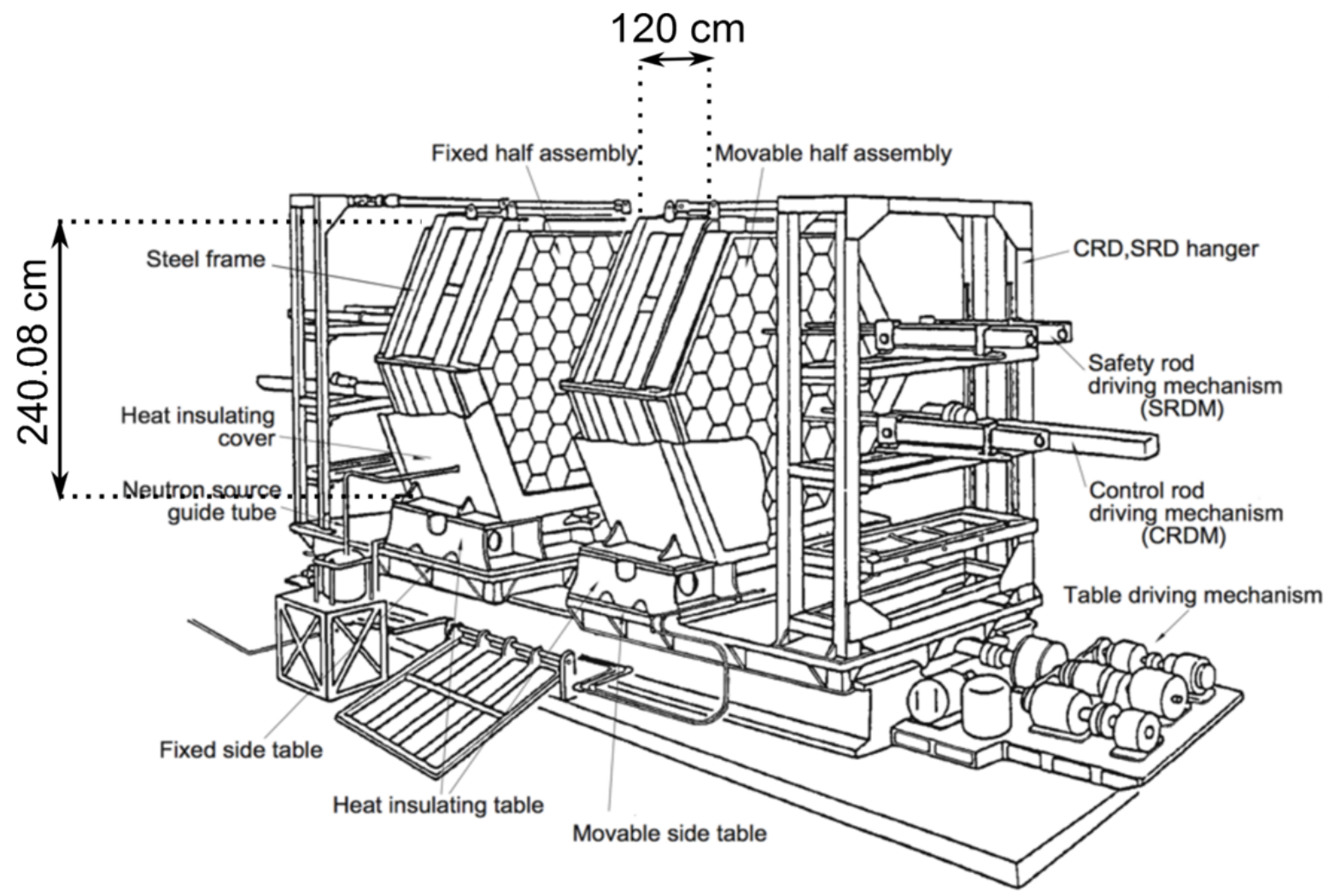

Figure 24. The VHTRC Assembly 23. 
The assembly consists of two axially jointed hexagonal-prism half assemblies of which one is fixed and the other is movable. The structures of the half assemblies are made of graphite blocks supported with steel frames. In general, unused holes of graphite blocks are filled with graphite rods.

The reflector blocks are classified into three types: (1) blocks having three large holes for the HTTR mock-up control rods and three small holes for multi-purpose usage, (2) blocks having one small hole for multi-purpose usage such as insertion of electric heaters, and (3) outermost blocks having one small hole.

The shape of the graphite blocks for the first two types are hexagonal, while the outermost blocks, which are located along the radial perimeter of the core, are trapezoidal to form a hexagonal assembly. The fuel block has 19 holes into which fuel rods, graphite rods, BP simulation rods, etc., can be inserted according to the experiment. Each fuel rod is paired with a solid graphite rod to reach an axial length of $1,200 \mathrm{~mm}$. The fuel rod consists of 20 fuel compacts, one graphite sheath, and two graphite end caps. The fuel rod is a stack of fuel compacts packed in a cylindrical graphite sheath. Two kinds of fuel rods were prepared: B-2 and B-4 rods containing fuel compacts of B-2 and B-4 types, respectively. The shape of a fuel compact is a hollow cylinder. The fuel compact is made of coated fuel particles uniformly dispersed in a graphite matrix. The coated fuel particles are bi-structural isotropic (BISO) particles (i.e., they have two carbon layers on a low-enriched uranium dioxide kernel). As mentioned above, there were two types of fuel compacts (B-2 and B-4 types) containing $2 \mathrm{wt} \%$ and $4 \mathrm{wt} . \%$ enriched uranium, respectively. Each fuel compact contains on average approximately 20,000 randomly distributed fuel particles.

The experiments were performed with three different loading patterns: HP, HC-1, and HC-2. The core loading pattern of the HP core is shown in Figure 25. This core consists mainly of B4-type fuel rods, only one graphite block contains B2-type fuel. The HC-1 core corresponds to the HP core with the B2-type fuel rods being replaced by graphite rods. Additionally, HC-2 core contains six B2-type fuel blocks compared to the HC-1 core. Furthermore a few additional B4-type fuel rods are placed in graphite blocks around the fuel blocks. In the experiments using the HP core, the assembly was first brought to critical state at room temperature $\left(25.5^{\circ} \mathrm{C}\right)$, and the critical point was determined using calibrated control rods. After that, the whole core was heated up to $200^{\circ} \mathrm{C}$ using electric heaters. In the course of core heating, the reactivity change owing to temperature rise was measured by the pulsed neutron method at different temperatures $\left(71.2,100.9,150.5\right.$, and $\left.199.6^{\circ} \mathrm{C}\right)$. In the experiments using the $\mathrm{HC}-1$ core, the critical point at $8.0^{\circ} \mathrm{C}$ was determined, while that at $200.3^{\circ} \mathrm{C}$ was determined for the $\mathrm{HC}-2$ core. Therefore, four critical and three subcritical configurations were obtained. All configurations have been evaluated as acceptable benchmark experiments.

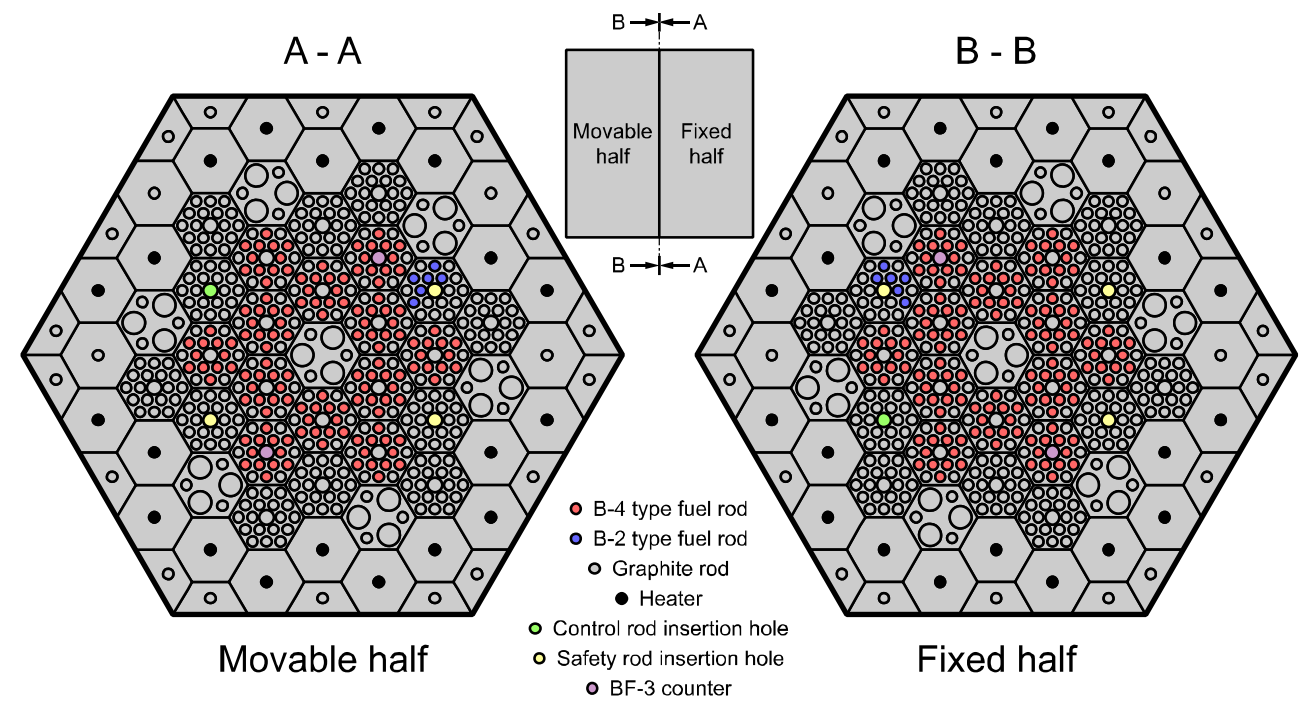

Figure 25. Core loading pattern of HP Core 23. 


\subsection{Computer Codes and Models}

The calculations of the VHTRC were performed with three different Monte Carlo codes: Serpent $2{ }^{23}$ MCNP5 ${ }^{24}$ and SCALE/KENO-VI. ${ }^{9}$ To perform Serpent calculations using the ENDF-B-VII.0 and ENDF-B-VII.1 cross-section data libraries, the slightly modified source code of Version 2.1.21 was used. In addition to simulations with SCALE 6.1.2, simulations with SCALE 6.2 beta Version 3 have been added (cf. Section 4.1).

\subsubsection{Serpent Model with a Random Particle Distribution}

The model is based on the benchmark specifications and an earlier INL Serpent model developed by J. Ortensi and H. Hammer (both at INL). It consists of the particles inserted in the fuel compacts/rods, the fuel rods arranged in fuel blocks, the fuel blocks arranged in a lattice, and two of these lattices combined to form the reactor core. Graphite rods and three different graphite blocks are included as well. Additionally, cadmium sheets and steel frames around the core are modeled (since cadmium is an important thermal neutron absorber, it was decided to model these layers, although the material composition are not specified in the description. Standard values are therefore assumed).

Serpent offers the opportunity to create a random particle distribution. For that purpose, the particle dimensions, the number of particles in one fuel rod, and the geometry of the fuel rod were submitted as input. Serpent then created a file containing the coordinates of the randomly distributed particles, which is included in the fuel rod. The specifications describe a small gap of less than $1 \mathrm{~mm}$ between the fuel/graphite rods and the surrounding graphite block. To simplify the model, this space is filled with block graphite. This simplification offers the opportunity to model the blocks containing only graphite rods as a solid graphite block, since the rod and the block material are identical. The graphite above and below the fuel rods can be modeled as a single block.

All carbon in all materials is treated as graphite (i.e., the thermal scattering data for graphite is applied in all carbonaceous materials). Furthermore, the thermal scattering data for uranium dioxide is applied and Doppler-broadening rejection correction for ${ }^{238} \mathrm{U}$ is considered. The black boundary condition removes all neutrons that leave the reactor. In addition to the HP core in Figure 25, cross-sectional views of core HC-1 and HC-2 are presented in Figure 26 and Figure 27.

For these models, 500,000 neutrons per cycle were calculated for 300 active cycles. The initial 40 cycles were skipped and not considered in the evaluation of the results. A combination 5 MPI combined with 31 OMP processes each led to simulation times of less than 4 hours.

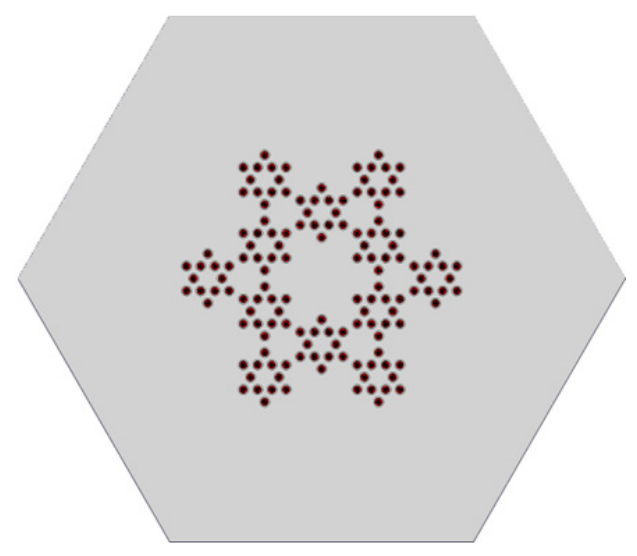

Figure 26. HC-1 core.

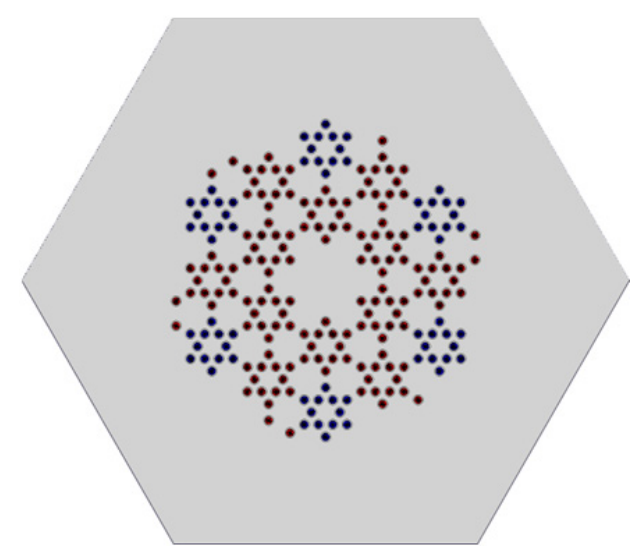

Figure 27. HC-2 core. 


\subsubsection{Serpent Model with a Particle Lattice}

In addition to the model with randomly-distributed particles, a second Serpent model was developed based on the MCNP model of J. Bess (INL). The particles in the fuel rod are arranged in a regular square lattice. One particle layer in a B2 fuel rod contains 667 particles, and one layer in B4 fuel contains 680 particles. A vertical stack of 30 particle layers forms one compact and 20 compacts form a fuel rod. Thus, vertical stacks of 600 particle layers were created and contained within the fuel rods. It is worth mentioning that the particles are arranged in a way that no particle is cut by the inner or outer fuel rod surface. As a result the local packing fraction in a particle lattice cell is slightly increased, and the specified packing fraction is only attained in the average packing fraction of one fuel compact. The same settings as in the random particle simulation were applied. The simulations were completed within 4.5 hours.

\subsubsection{MCNP5 and KENO-VI Models}

The MCNP and KENO-VI models are identical to the Serpent lattice model. For the KENO-VI MG calculations, the DOUBLEHET cell data was specified to include the double heterogeneous structure of the BISO particles in the fuel compact.

The SCALE simulations included 50,000 neutrons in 500 active cycles and 50 initially skipped cycles. On a single processor, the CE KENO-VI simulations took about 25 hours and the MG calculations less than 8 hours.

Due to differences between the multiplication factors determined by the three codes, additional simulations were performed using one B2 particle layer in a hexagonal cell (Figure 28) and a B2 fuel rod unit cell in a hexagonal prism (Figure 28 to Figure 31 ) with reflective boundary conditions.

It should also be noted that the particle lattice of B2 is not symmetric, and the orientation of the lattice in the compact might have a small influence on the result. The lattice models of the calculations reported here are checked for consistency. Furthermore, all Serpent and MCNP calculations are performed using both the ENDF-B-VII.0 and the ENDF-B-VII.1 cross-section libraries. The ENDF-B-VII.0 library is the only option currently available in SCALE/KENO-VI.

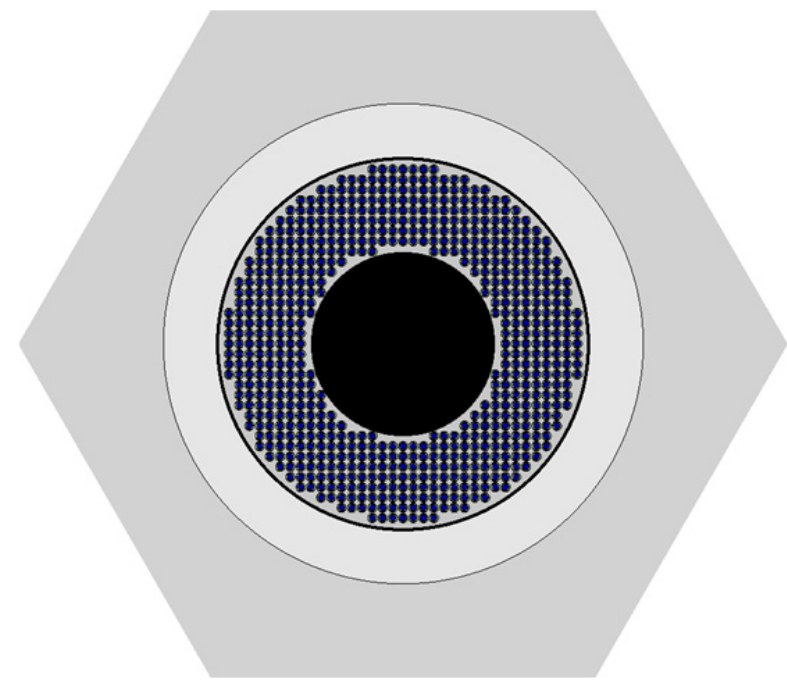

Figure 28. Cross-sectional view of B2 fuel unit cell with a particle lattice (one particle layer).

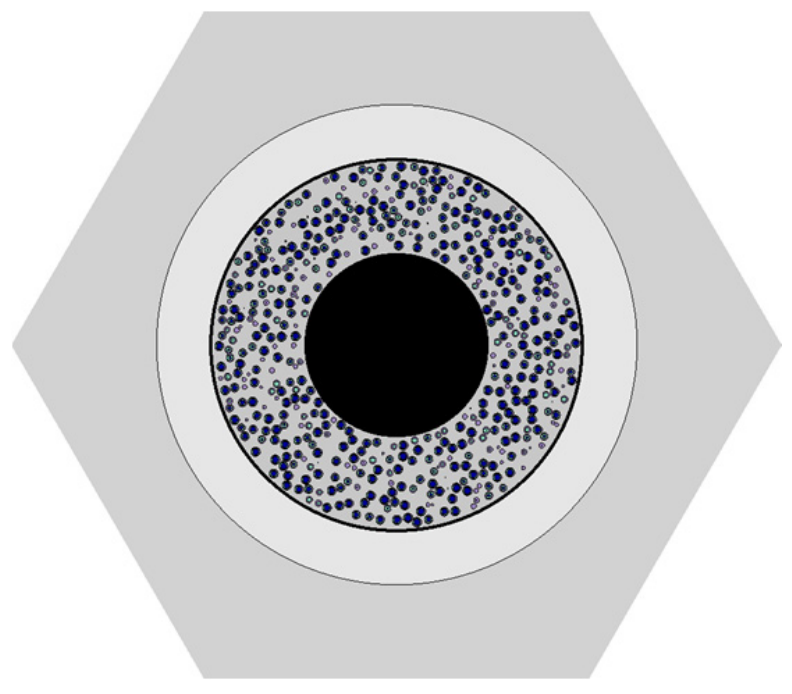

Figure 29. Cross-sectional view of a B2 fuel unit cell with a random particle distribution. 


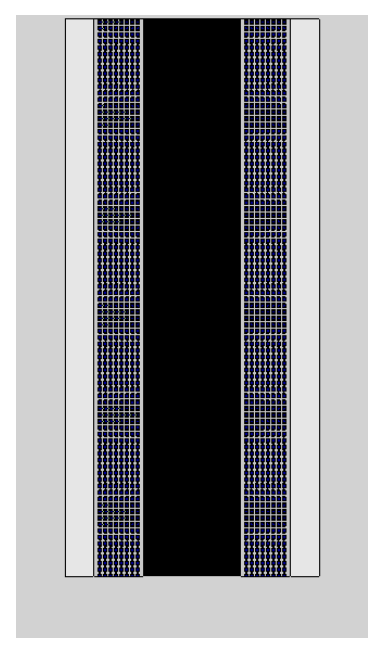

Figure 30. Vertical cross section of a B2 fuel unit cell with a particle lattice (not to scale).

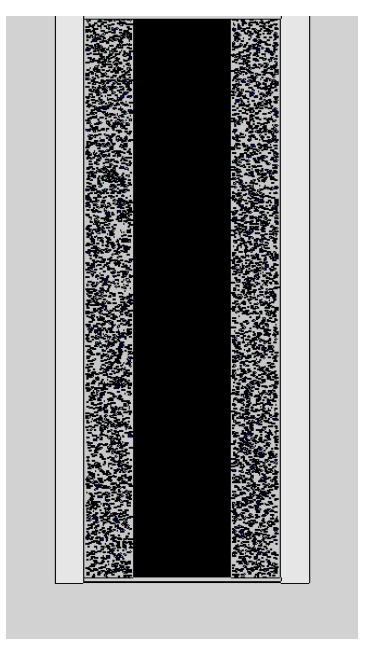

Figure 31. Vertical cross section of a B2 fuel unit cell with a random particle distribution (not to scale).

\subsection{Comparison of VHTRC Results}

\subsubsection{Convergence Behavior}

To discard a sufficient number of initial neutron cycles in the Monte Carlo simulations, the convergence of the multiplication factor and the Shannon entropy of the fission source distribution were investigated. Since the KENO-VI simulations were performed with 50,000 neutrons per cycle, the multiplication factor and the Shannon entropy are shown in Figure 32 and Figure 33, respectively, as a function of the first 100 neutron cycles with 50,000 neutrons each. All figures show that both the multiplication factor and the entropy have already converged before the fiftieth neutron cycle. Therefore, it is sufficient to discard the first 50 cycles. The Serpent and MCNP calculations were performed with 500,000 neutrons per cycle. Thus the multiplication factor and the entropy converge earlier than in the KENO-VI calculations and fewer cycles can be skipped.

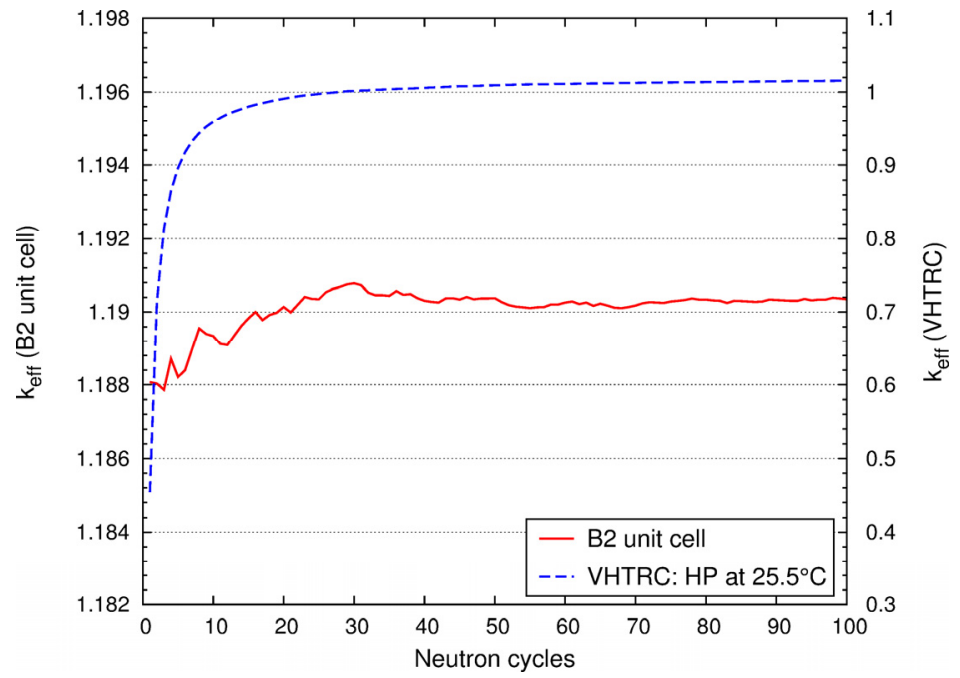

Figure 32. Multiplication factor as a function of neutron cycles with 50,000 neutrons/cycle for the B2 unit cell and the VHTRC HP core at $25.5^{\circ} \mathrm{C}$. 


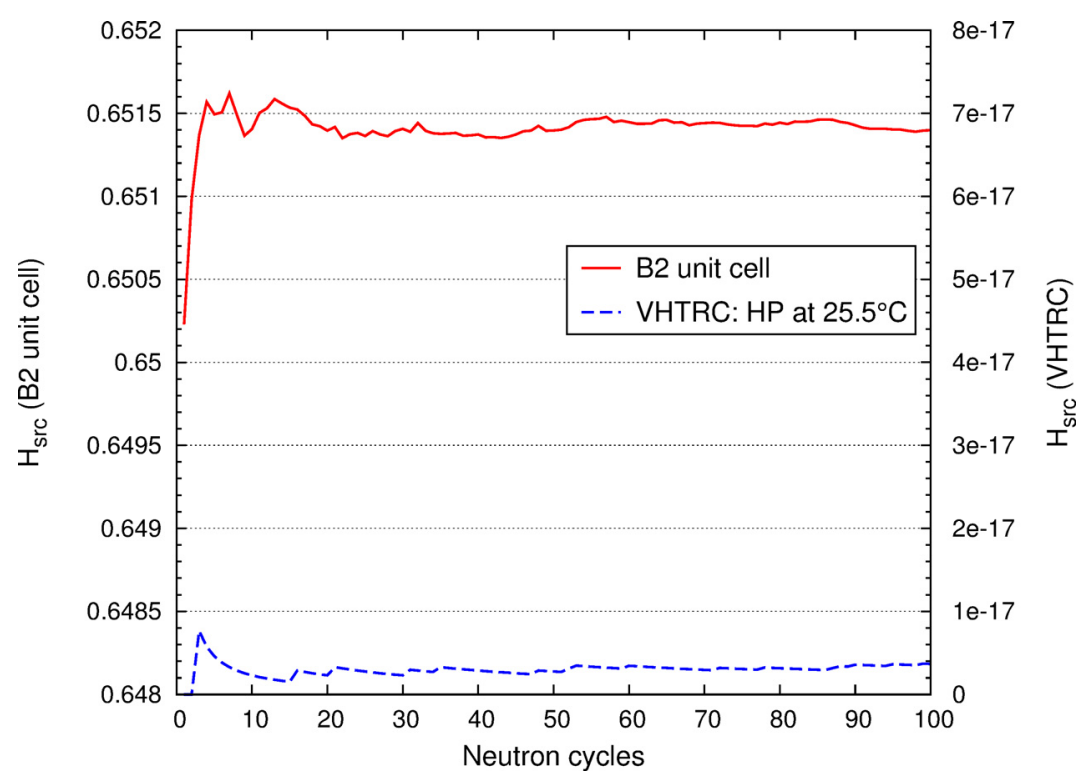

Figure 33. Shannon entropy of the fission source distribution as a function of neutron cycles with 50,000 neutrons/cycle for the B2 unit cell and the VHTRC HP core at $25.5^{\circ} \mathrm{C}$.

\subsubsection{Influence of the KENO-VI/DOUBLEHET Cell Treatment}

The DOUBLEHET treatment in KENO-VI does not allow annular fuel compacts. In simulations of the HTTR, Ilas ${ }^{22}$ and Wang ${ }^{16}$ homogenized the fuel compact with their central hole. To figure out the most appropriate cell treatment, calculations with four different DOUBLEHET cell specifications were performed as part of this study:

1. The annular fuel compact was homogenized with the inner void. As a result, the volume of the fuel region was increased and the packing fraction of the BISO particles in the graphite matrix was decreased.

2. The annular fuel compact was condensed to a solid cylinder with a smaller radius to preserve the correct volume of the fuel region and the correct packing fraction.

3. The number of particles was increased to retain the outer radius of the fuel compact and the correct packing fraction.

4. The same cell version as Case 1 , but with the geometry changed to a solid cylinder (in contrast to Cases 1-3, where the annular geometry was used). This fourth option seems to correspond to the homogenization approach of Ilas and Wang.

All simulations were performed for both the B2 fuel unit cell model and the full VHTRC block. The resultant multiplication factors are compared with the respective CE lattice calculations in Table 19. Cases 1 and 4 resulted in the largest deviations from the CE result $(>1,000 \mathrm{pcm})$. Case 2 is closer to the $\mathrm{CE}$ result, especially in the block calculation. Case 3 produced the best results for both models. Therefore, the cell specifications of Case 3 were chosen for all subsequent KENO-VI MG calculations. 
Table 19. Comparison of different KENO-VI/DOUBLEHET cell treatments.

\begin{tabular}{|c|c|c|c|c|}
\hline \multicolumn{3}{|c|}{ Model } & $\mathrm{k}_{\mathrm{eff}} \pm \sigma$ & $\begin{array}{l}\Delta \pm \sigma \\
{[\mathrm{pcm}]}\end{array}$ \\
\hline \multirow{5}{*}{ 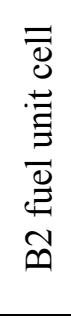 } & \multicolumn{2}{|c|}{ KENO-VI CE (6.1.2) - lattice } & $1.19387 \pm 0.00024$ & (reference) \\
\hline & \multirow{4}{*}{$\begin{array}{l}\text { KENO-VI } 238 \mathrm{MG} / \\
\text { DOUBLEHET (6.1.2) }\end{array}$} & Case 1 & $1.21592 \pm 0.00017$ & $2205 \pm 32$ \\
\hline & & Case 2 & $1.18507 \pm 0.00018$ & $-880 \pm 30$ \\
\hline & & Case 3 & $1.18938 \pm 0.00017$ & $-449 \pm 30$ \\
\hline & & Case 4 & $1.18403 \pm 0.00018$ & $-984 \pm 30$ \\
\hline \multirow{5}{*}{ 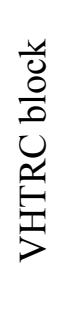 } & \multicolumn{2}{|c|}{ KENO-VI CE (6.1.2) - lattice } & $1.02914 \pm 0.00018$ & (reference) \\
\hline & \multirow{4}{*}{$\begin{array}{l}\text { KENO-VI } 238 \mathrm{MG} / \\
\text { DOUBLEHET (6.1.2) }\end{array}$} & Case 1 & $0.98303 \pm 0.00015$ & $-4611 \pm 24$ \\
\hline & & Case 2 & $1.02462 \pm 0.00018$ & $-452 \pm 26$ \\
\hline & & Case 3 & $1.02600 \pm 0.00017$ & $-314 \pm 25$ \\
\hline & & Case 4 & $0.98306 \pm 0.00015$ & $-4608 \pm 24$ \\
\hline
\end{tabular}

The approach followed for Case 4 seems not to be appropriate for the simulation of the VHTRC. A reason might be that the ratio between the volume of the central hole and the fuel compact volume is nearly twice as large in the VHTRC as in the HTTR. Ilas found a difference of $68 \mathrm{pcm}$ in CE calculations between an explicit annular ring and a homogenized fuel pin, but similar CE calculations of a homogenized ring in the VHTRC led to larger differences of $270 \mathrm{pcm}$ in the unit cell and $800 \mathrm{pcm}$ in the VHTRC block. (Although it is not explicitly mentioned, the so-called homogenization was interpreted to be a dispersion of the same number of particles as in the annular ring but in the solid cylinder. Therefore, the packing fraction is decreased.). The impact on the packing fraction and the inner void might thus have a larger significance in the simulations of the VHTRC.

\subsubsection{B2 Particle Layer and Fuel Unit Cell}

Simulations of one B2 particle layer in a hexagonal cell with Serpent, MCNP5, and SCALE 6.1.2 CE showed consistent multiplication factors with overlapping error bars. The MG calculations underestimate the CE multiplication factors by several hundred pcm, and the SCALE 6.2b3 CE calculation overestimates $\mathrm{k}_{\infty}$ by about $140 \mathrm{pcm}$.

For the B2 fuel unit cell calculations presented in Table 20, the Serpent calculation using a random particle distribution was chosen as the reference. The Serpent lattice calculation showed an overestimation of $\mathrm{k}_{\infty}$ of more than $500 \mathrm{pcm}$. This overestimation might arise from a reduced average distance between the fuel particles in the fuel compacts compared to a random distribution. 
Table 20. Multiplication factor of a B2 fuel unit cell.

\begin{tabular}{|l|c|c|}
\hline \multicolumn{1}{|c|}{ Model } & \multicolumn{1}{c|}{$\mathrm{k}_{\infty} \pm \sigma$} & $\begin{array}{c}\Delta \pm \sigma \\
{[\mathrm{pcm}]}\end{array}$ \\
\hline Serpent - random (ENDF-B-VII.0) & $1.19034 \pm 0.00005$ & (reference) \\
\hline Serpent - random (ENDF-B-VII.1) & $1.17677 \pm 0.00005$ & $-1357 \pm 7$ \\
\hline Serpent - lattice (ENDF-B-VII.0) & $1.19541 \pm 0.00004$ & $507 \pm 7$ \\
\hline Serpent - lattice (ENDF-B-VII.1) & $1.18189 \pm 0.00005$ & $-845 \pm 7$ \\
\hline MCNP5 - lattice (ENDF-B-VII.0) & $1.19487 \pm 0.00005$ & $453 \pm 7$ \\
\hline MCNP5 - lattice (ENDF-B-VII.1) & $1.18131 \pm 0.00005$ & $-903 \pm 7$ \\
\hline KENO-VI CE -lattice (6.1.2) & $1.19387 \pm 0.00024$ & $353 \pm 25$ \\
\hline KENO-VI CE -lattice (6.2b3) & $1.19609 \pm 0.00022$ & $575 \pm 23$ \\
\hline KENO-VI 238 MG/DOUBLEHET (6.1.2) & $1.18938 \pm 0.00017$ & $-96 \pm 18$ \\
\hline KENO-VI 238 MG/DOUBLEHET (6.2b3) & $1.19012 \pm 0.00022$ & $-22 \pm 23$ \\
\hline KENO-VI 252 MG/DOUBLEHET (6.2b3) & $1.18774 \pm 0.00019$ & $-260 \pm 20$ \\
\hline
\end{tabular}

The MCNP calculation reveals a similar deviation from the reference result. However, a difference of about $50 \mathrm{pcm}$ compared to the Serpent lattice calculation is visible where a very close match (overlapping error bars) was expected. To exclude an influence of the outermost, reflecting hexagonal surface, calculations with an outer cuboid surface were performed. Furthermore, a stack of 600 particle layers with $100 \mathrm{~cm}$ of graphite above and below the stack was also modeled to exclude influences of the actual unit cell geometry. However, all calculations showed deviations between MCNP5 and Serpent in the same order $(50 \mathrm{pcm})$. The cause of these differences is not yet completely understood, and will been investigated at a later stage.

The CE calculation with SCALE 6.1.2 showed a multiplication constant closer to the reference than the respective Serpent and MCNP particle lattice calculations. However, the $\mathrm{k}_{\infty}$ is about $150 \mathrm{pcm}$ lower than in the Serpent lattice calculation (i.e., a Serpent calculation including the same particle model). The MG calculation underestimates the reference result by about $100 \mathrm{pcm}$ (i.e., it is more than $500 \mathrm{pcm}$ lower than the CE calculation).

The SCALE 6.2b3 CE calculation showed a slightly closer result to the respective Serpent lattice calculation with a difference of about $70 \mathrm{pcm}$. The 238 -group calculation is also closer to the reference than the respective SCALE 6.1.2 calculation. However, the 252-group calculation underestimates the reference by $260 \mathrm{pcm}$.

All calculations of ko using the ENDF-B-VII.1 cross-section library are lower than the reference result. The differences to the respective calculations using ENDF-B-VII.0 are around 1,300 pcm in all cases. These high differences are assumed to be caused by the correction of the neutron capture cross section in graphite. Because of a larger neutron capture in ENDF-B-VII.1, the multiplication factor is reduced 14. The difference of the microscopic capture cross sections of carbon between the libraries is shown in Figure 34, where the ENDF-B-VII.1 library shows a thermal cross section approximately $0.4 \mathrm{mb}$ larger at $0.025 \mathrm{eV}$ than in the ENDF-B-VII.0 library. 


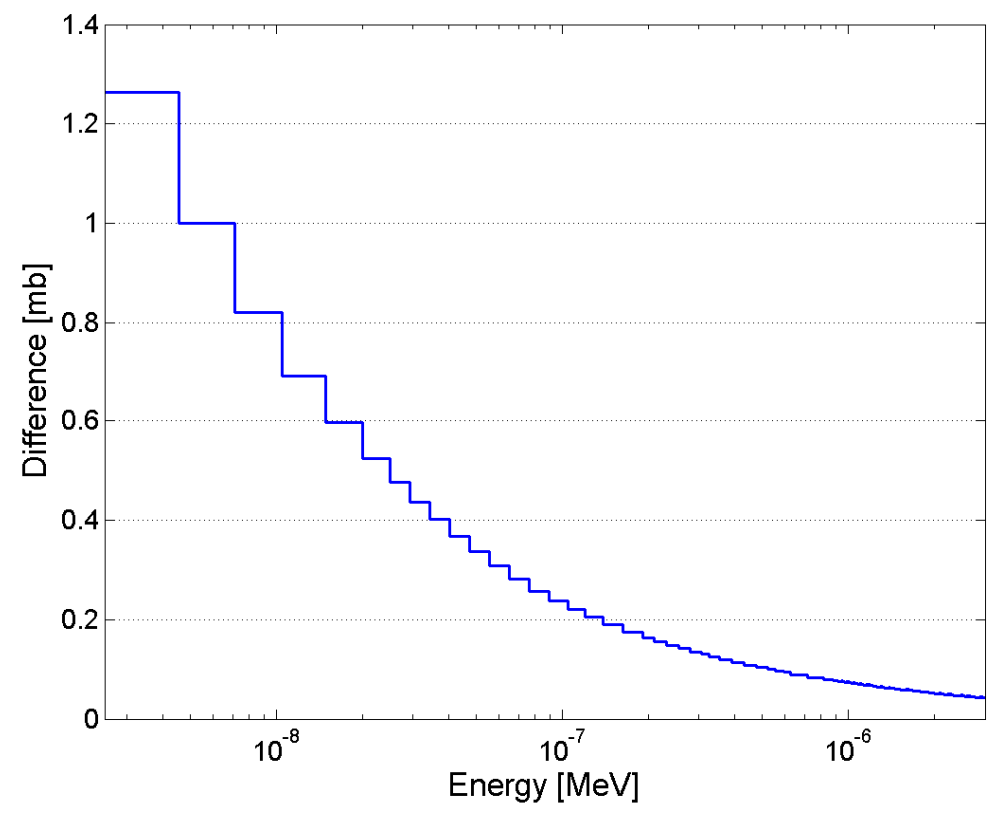

Figure 34. Difference $(\mathrm{mb})$ of the microscopic capture cross section of carbon at $293.6 \mathrm{~K}$ between the ENDF-B-VII.1 and ENDF-B-VII.0 library.

\subsubsection{VHTRC}

The simulations of the full block with MCNP5 could not yet be completed. The error "zero lattice element hit" occurs after the calculation of a number of calculated neutron cycles, stopping the calculation. Some of the errors could be avoided by changing the box in which one particle is located from a definition via macro bodies to explicit surface definitions. The size of the lattices was also increased by adding some graphite cells on all sides to avoid the lattice universe border matching the surface border of the universe. However, these simulations also failed before all cycles were calculated. Furthermore, it is not possible to avoid a model with particle lattices by using cell or surface transformation cards. These cards are limited to 999 transformations. However, both fuel types require a single particle layer with more than 600 particles each. A model with both fuel types is not possible (without changes in the code). An explicit formulation of every single particle already led to segmentation faults in small models. Therefore, no reliable MCNP results can be presented at this point.

In addition to the multiplication factor, the neutron flux was examined. The flux spectra of the VHTRC block and a B2 unit cell are shown in Figure 35. Due to the large amount of graphite (i.e., a large moderator-fuel ratio), the thermal peak in both models is distinctly higher than the corresponding fast peak). As is already observed in the discussion of the MHTGR-350 results in the previous section, the peak in the full block is higher than in the unit cell because of a larger moderator-fuel ratio in the block. 


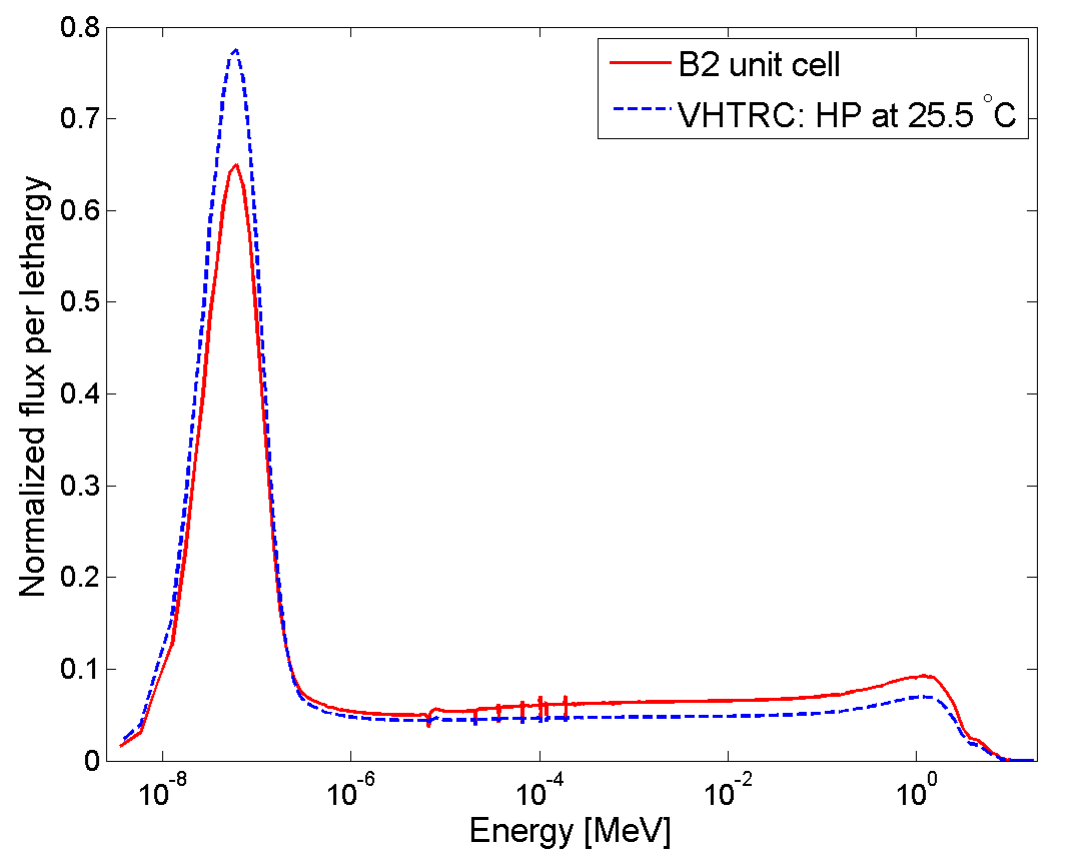

Figure 35. Normalized neutron flux in the B2 fuel unit cell and VHTRC HP core at $25.5^{\circ} \mathrm{C}$.

The multiplication factors of all calculated states and cores are compared as a function of temperature in Figure 36 to Figure 41. All figures are subdivided into three parts to present the three cores next to one other. The experimental values are shown in all plots as well, and the results of the Japanese MVP-II calculations, as reported in the VHTRC specifications 23 are added in Figure 40 and Figure 41.

The Serpent calculations show differences of $120-160 \mathrm{pcm}$ in the HP and HC-1 cores and about $200 \mathrm{pcm}$ in the HC-2 core between the random and the regular particle distributions for both libraries (Figure 36). The HC-2 core contains more fuel than the other cores, and so the influence of the fuel particle distribution might be more important. The decreased influence of the particle distribution agrees well with the MHGTR-350 simulations, where the largest geometry revealed the smallest differences between the random and the regular particle distribution. The general decrease of keff with increasing temperature can mainly be explained by Doppler resonance broadening of ${ }^{238} \mathrm{U}$.

The calculations with ENDF-B-VII.0 cross sections show differences of up to 1,200 pcm compared to the experiment. The corresponding ENDF-B-VII.1 calculations show significantly closer results to the experiment (within the large error bars of the experiment), which can be seen as an indication that the older version of the ENDF-VII library is not suitable for graphite HTGR systems. 


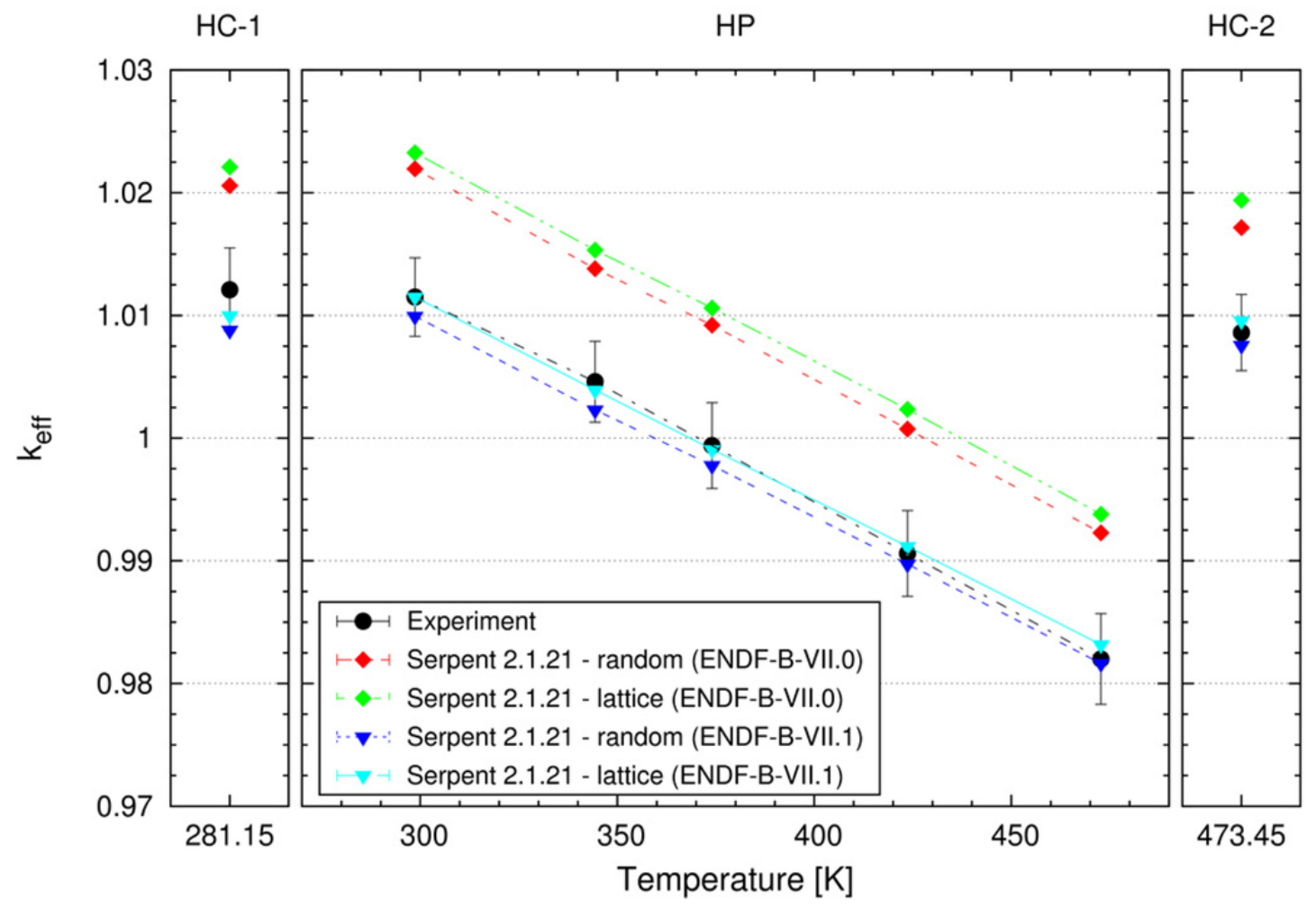

Figure 36. Comparison of Serpent 2 and experimental VHTRC multiplication factors.

SCALE 6.1.2 is not capable of adjusting temperatures in CE calculations, and the program therefore uses cross-section data from the closest available temperature point in the library. As a result, the multiplication factors of the two coldest HP cores are within one standard deviation of each other (Figure 37). These calculations only differ in the $1 \mathrm{H}$ temperature used, which does not have an important influence. The next two cores share the same graphite temperature of $400 \mathrm{~K}$, which show similar results. The decrease in keff to the hottest HP core is the result of the temperature jump of graphite to $500 \mathrm{~K}$ and in all other nuclides (except for $1 \mathrm{H}$ ) from $293.6 \mathrm{~K}$ to the next available library data point at $600 \mathrm{~K}$. The influence of the resonance broadening is clearly visible. The simulation of the HC-1 core is identical to the coldest HP core except for the reduced amount of fuel that causes a slight reduction in keff.

In SCALE 6.2b3 problem-dependent Doppler-broadening was applied, ${ }^{30}$ and the corresponding CE calculations therefore show a continuous decrease with increasing temperature. The SCALE 6.2b3 results are furthermore closer to the corresponding Serpent model (particle lattice, ENDF-B-VII.0 cross sections) in most cases with differences of $190-450 \mathrm{pcm}$. This is the result of various improvements such as the $\mathrm{S}(\alpha, \beta)$ treatment and the application of DBRC in the latest SCALE version. ${ }^{29}$ Since only the ENDF-B-VII.0 library can be applied in SCALE at this stage, all results show significant differences with the experimental values. 


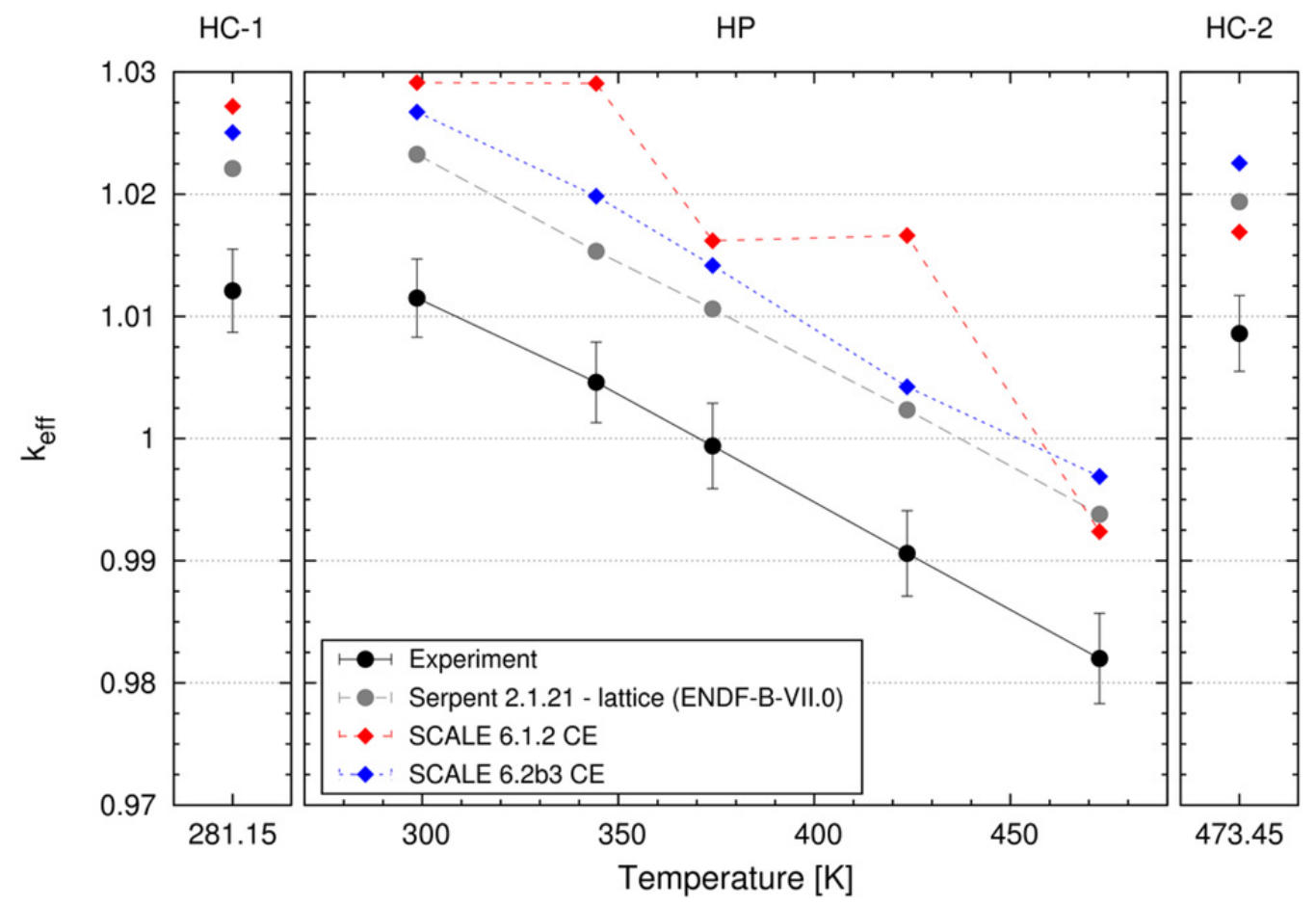

Figure 37. Comparison of SCALE/KENO-VI CE, Serpent and experimental VHTRC multiplication factors.

KENO-VI MG uses an interpolation procedure to calculate the corrected cross sections at the requested temperatures. As a result, the multiplication factor of the HP core is consistently reduced as the temperature increases (Figure 38). The calculations with 238 groups have overlapping error bars in most cases. All MG calculations tend to show smaller multiplication factors than the corresponding CE calculations.

The 252 group calculation with SCALE $6.2 \mathrm{~b} 3$ reveals an even smaller keff than the 238 group calculations and is therefore closer to the reference Serpent random calculation. However, the gradient of the 252 group curve connecting the HP cores is slightly different than in the 238 group calculations. In contrast to the 252 group results, the 238 group calculations show nearly linear behavior. Since these SCALE $6.2 \mathrm{~b} 3$ simulations differ only in the applied group structure, this difference is suggested to be a result of differences in the library. For testing purposes the HP cores have been calculated with natural carbon instead of graphite (i.e., without thermal scattering in graphite). As expected, these multiplication factors show large differences to the corresponding graphite calculations, and the temperature gradient is different. However, there is no over-average drop between two values. Therefore, differences in the thermal scattering library between the 238- and 252-group structure are suggested to cause the respective drop in Figure 38.

In general, all SCALE MG results are significantly higher than the experiment due to the Version 0 of the ENDF-B-VII library. The applied DOUBLEHET cell is also not annular, and was based on a set of MG calculations with various cells as a subjective modeling choice. Therefore, these results might include a systematic error in addition to the library effect. 
The differences of all SCALE calculations to the reference are presented in Figure 39. The improvements of the SCALE 6.2b3 values compared to SCALE 6.1.2 calculations are clearly visible. As already observed in the MHTGR calculations, the 252-group calculations show the closest results to the reference for most cases. The only exceptions to this trend are the SCALE 6.1.2 CE calculations at certain temperatures that utilize inappropriate temperatures and therefore over estimate Doppler-broadening.

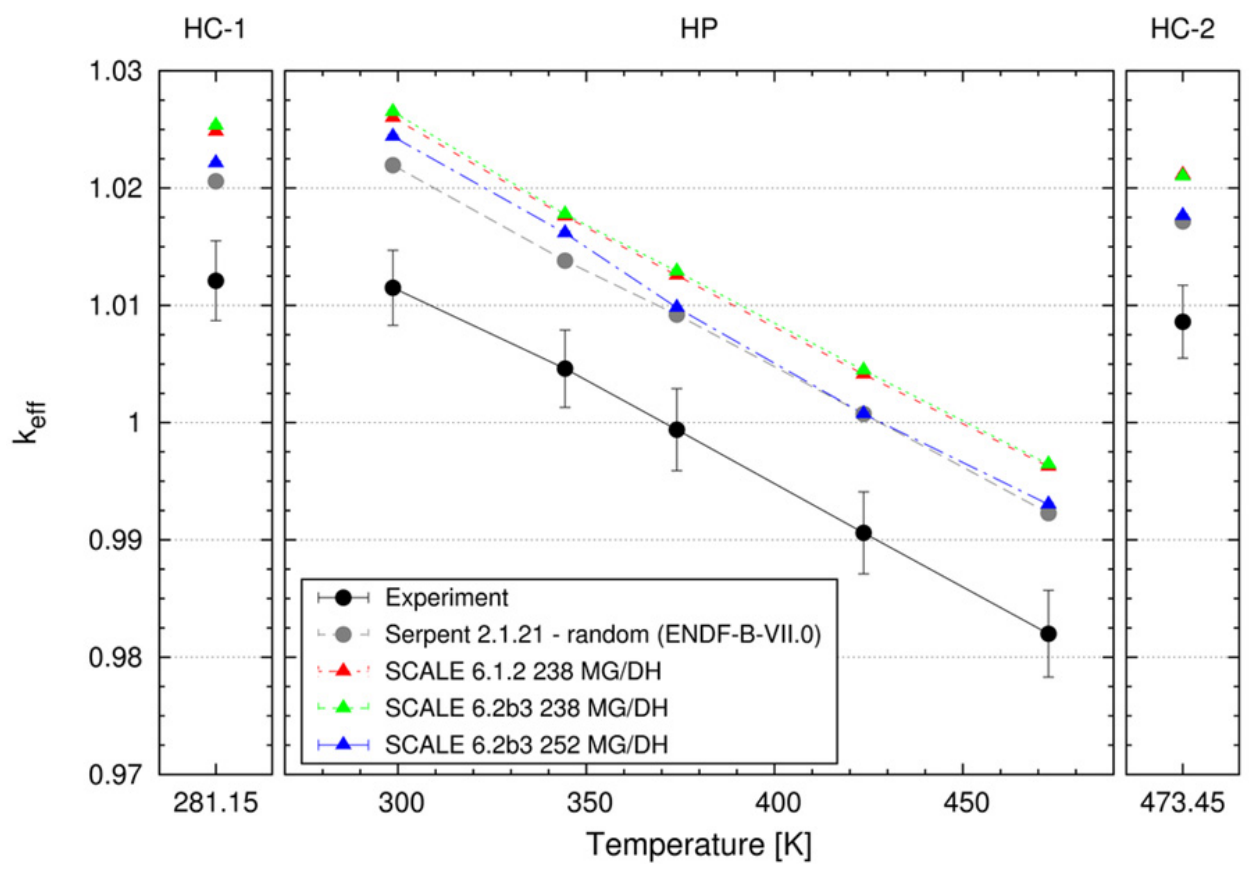

Figure 38. Comparison of the SCALE/KENO-VI MG and experiemntal VHTRC multiplication factors.

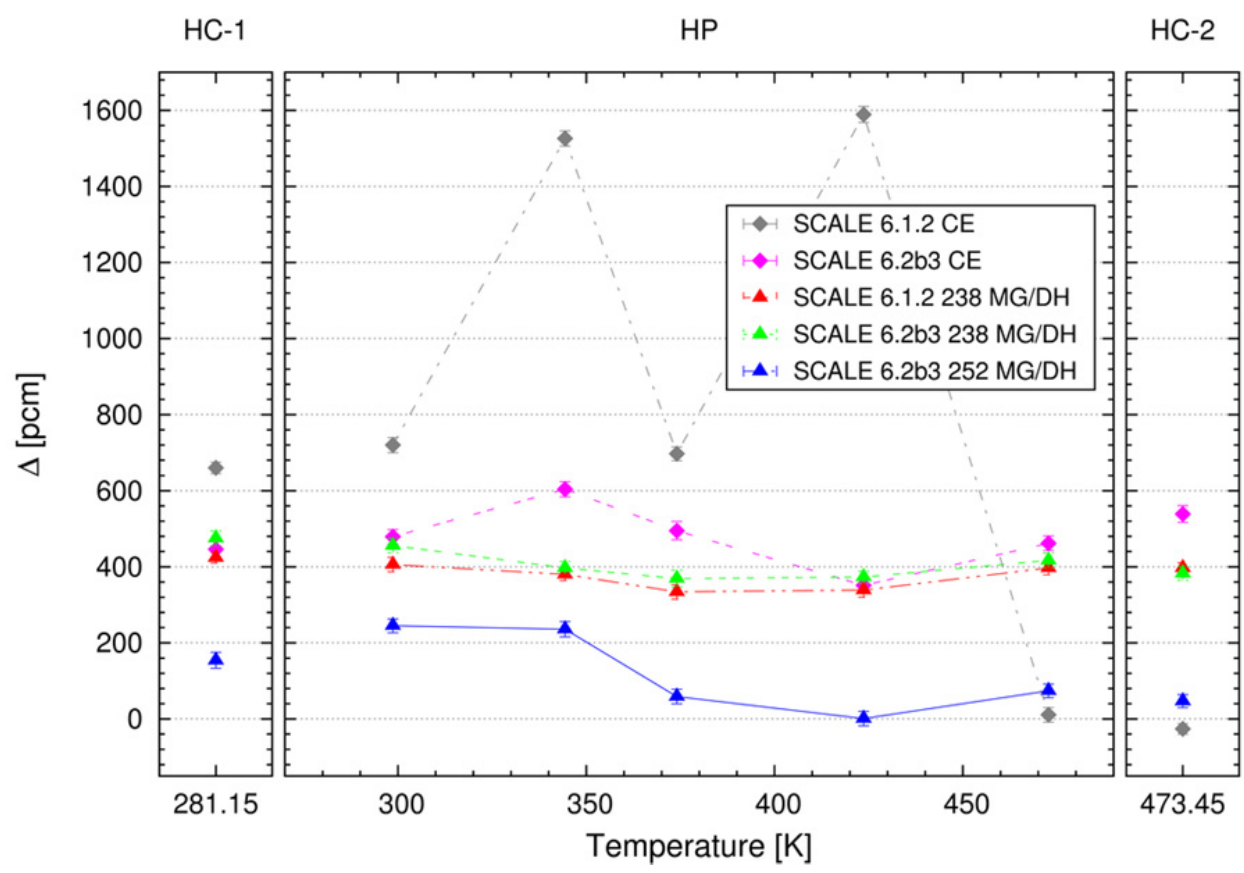

Figure 39. Difference $(\mathrm{pcm})$ between the SCALE/KENO-VI and the reference Serpent random (ENDF-B-VII.0) multiplication factor of the VHTRC. 
A comparison of the Japanese MVP-II calculations (as published in the specifications of the VHTRC $^{23}$ ) show a consistent underestimation of the experimental values by $300-500 \mathrm{pcm}$ for the HP and HC-2 cores, and by about $700 \mathrm{pcm}$ for the HC-1 core. The MVP-II data sets are compared to the Serpent calculations using the ENDF-B-VII.1 data library and random particle distributions in Figure 40, because the neutron absorption cross section of graphite has also been corrected in the JENDL-4.0 Library14 and the MVP-II model utilized a random particle distribution. The difference between the Serpent calculations and the MVP results is additionally shown in Figure 41 for two variations on the INL VHTRC model utilized for these CRP calculations. As mentioned earlier, the INL CRP model of the VHTRC fills the gaps between the fuel rods and the graphite block with graphite. Therefore, the amount of graphite is slightly increased and moderation improved. A better comparison to the MVP calculation is achieved with a model that includes these gaps. It is shown in Figure 41 that the inclusion of the gaps leads to multiplication factors about $200 \mathrm{pcm}$ smaller and closer to the MVP model. It was also noticed that the material specifications used in the MVP model are slightly different from the final benchmark specifications, so a third set of calculations was performed with the MVP-II material data. The result are multiplication factors up to $80 \mathrm{pcm}$ smaller in the HP and $\mathrm{HC}-1$ core, and about $100 \mathrm{pcm}$ smaller in the HC-2 core. Three of the core states now produced results with overlapping error bars with the corresponding MVP results. Since the differences with the MVP results are not constant, although the HP cores differ only in temperature, differences in the applied libraries are assumed.

The Serpent model utilizing a random particle distribution the latest release of the ENDF crosssection library and with gaps included is supposed to represent the closest model to the benchmark specifications. However, this model still shows differences of a few hundred pcm to the experiment. Some multiplication factors lie within the error bars of the experiment, but these error bars are relatively large at approximately $350 \mathrm{pcm}$. The remaining differences might, among other factors, be caused by material simplifications. The materials of the graphite block and rods have been combined, and the effects of different impurities or densities for these two materials could not be considered. Furthermore, the provided MVP-II input suggests that the material definitions have been slightly changed during the development of the benchmark specifications by decreasing the original number densities from 6 digits to 4 digits.

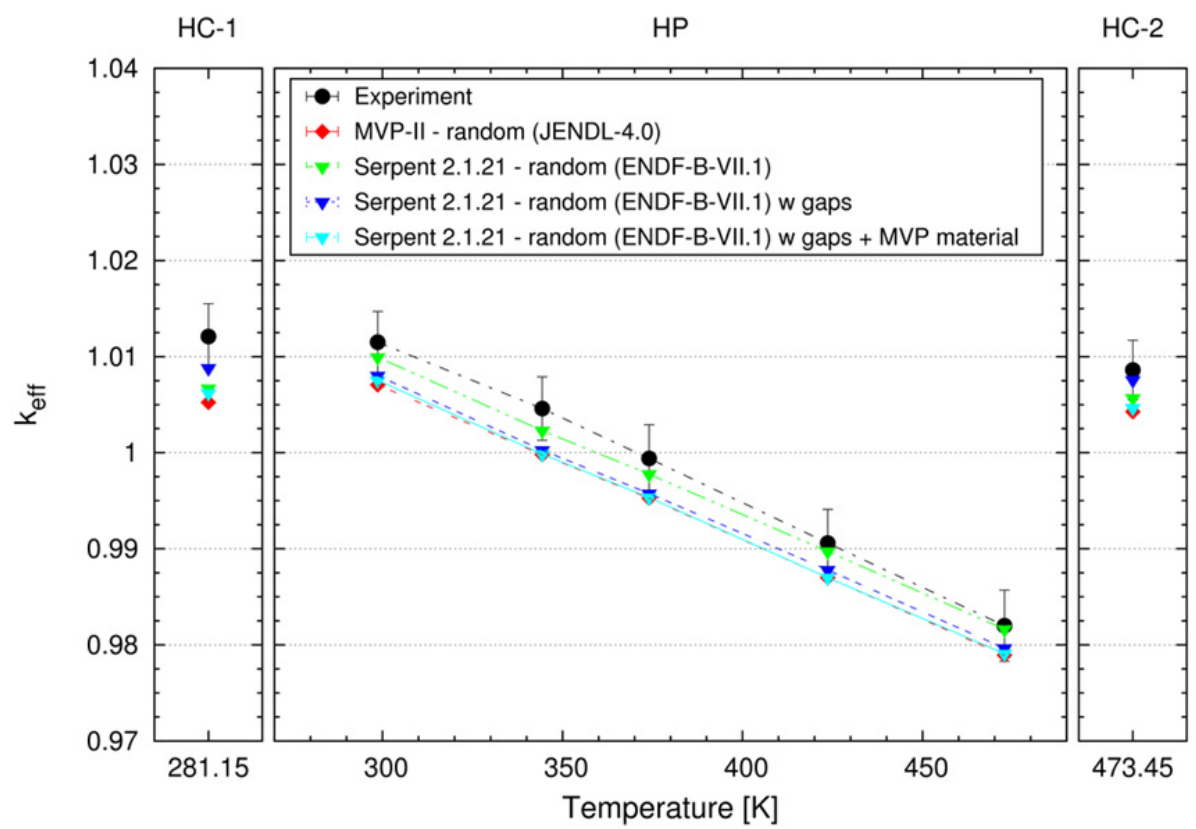

Figure 40. Comparison of the Serpent 2 (ENDF-B-VII.1) and MVP-II (JENDL-4.0) VHTRC multiplication factors. 


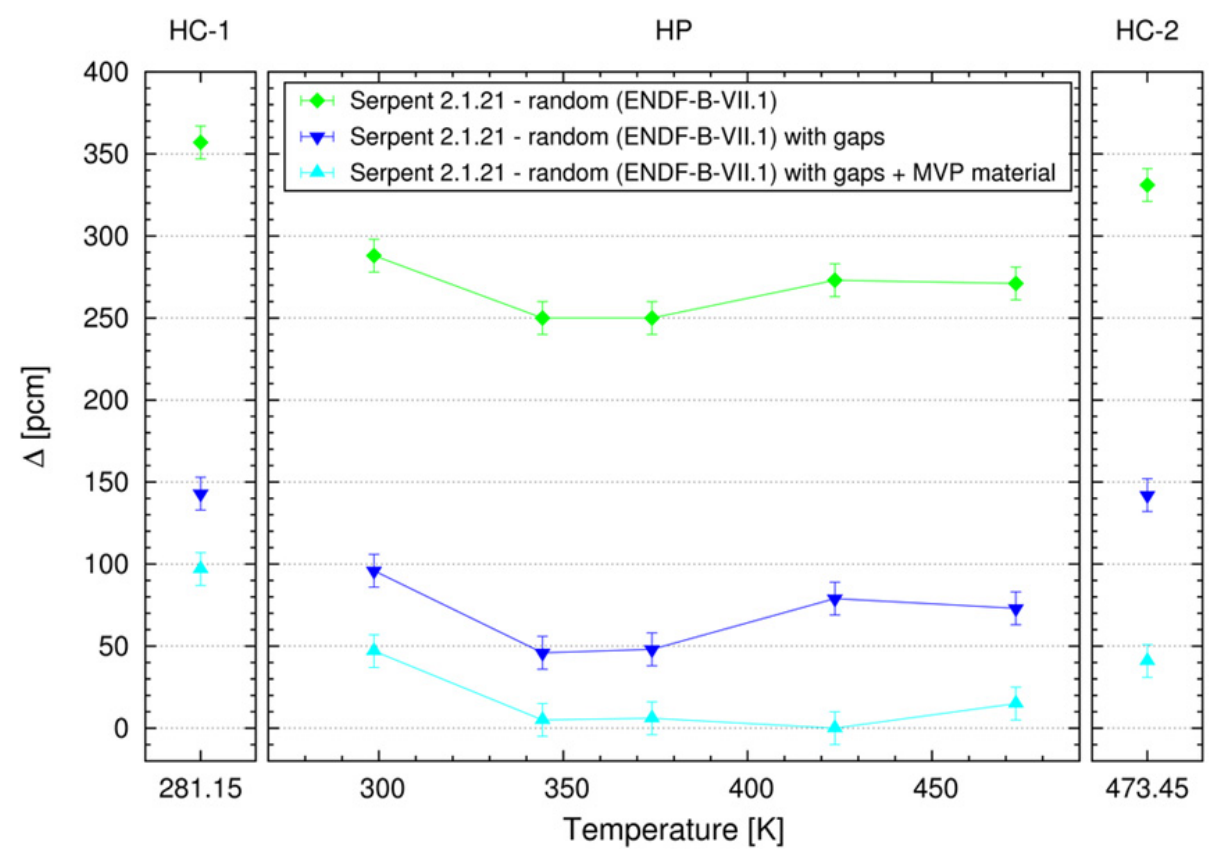

Figure 41. Differences (pcm) between VHTRC multiplication factors of the CRP model and variations including gaps and different material compositions.

\subsection{VHTRC Summary}

For the validation of the cell and lattice calculations of Exercises I-1 and I-2 in Phase I, criticality simulations of the VHTRC were performed with the Monte Carlo codes MCNP5, Serpent 2, and SCALE/KENO-VI. In addition to calculations of the fuel block, simulations of a fuel unit cell have been investigated.

The comparison of a unit cell and the fuel block revealed an influence of the model size on the simulation results. The Serpent calculations showed that the difference between the simulations of a random particle distribution compared to a regular particle lattice decreases as the model is expanded to include are larger portion of the physical domain. Furthermore, the difference between the SCALE 6.1.2 $\mathrm{CE}$ and $\mathrm{MG}$ calculations decreases when the model size is increased. The deviation of the multiplication factors of SCALE 6.1.2 compared to the Serpent calculations is increased at the same time.

SCALE 6.1.2 is not capable of adjusting the cross-section data to the requested temperature in CE calculations. A significant improvement in comparisons with Serpent and MG results was observed in CE calculations with SCALE 6.2b3, which considers the requested temperatures. All SCALE simulations are in excess of $1 \%(1,000 \mathrm{pcm})$ higher than the experimental data, since only ENDF-B-VII.0 cross sections can be applied. The SCALE 6.2b3 CE calculation showed better agreement with the corresponding Serpent lattice calculation than the SCALE 6.1.2 CE calculation due to various improvements in the new version. The 252-group calculation produced the best result when compared to either Serpent calculations containing regular or randomly distributed particles.

The Japanese MVP-II calculations revealed a consistent underestimation of the experiment and all INL simulations performed for this CRP. The addition of the gaps between the fuel and graphite rods and the surrounding graphite block in the model, and the use of the MVP-II material compositions with ENDF-B-VII.1 cross sections led to good agreement between the MVP-II and the Serpent random calculations. (The gaps and slightly modified material compositions are not part of the CRP or IHRP VHTRC specifications). 
The Serpent calculations using the ENDF-B-VII.1 library and a random fuel particle model produced the closest results to experimental values. The increase of the neutron capture cross section in graphite in this library compared to its prior release has a crucial impact on the calculations of the multiplication factor of this graphite moderated assembly. The use of this model as the reference for comparison in the preceding MHGTR-350 section is justified by these validation calculations.

\section{EXERCISES I-3 AND I-4: LOCAL THERMAL-HYDRAULIC COUPLING}

The final two exercises of Phase I are focused on the localized stand-alone fuel thermal response. The aim of the stand-alone thermal unit cell calculations is to isolate the effect of material and boundary input uncertainties on very simplified problems, before the same input variations are applied to complex core problems (Phases II-IV in Section 3). Exercise I-3 requires a steady-state solution for a single fuel compact and coolant channel unit cell with a fixed bulk coolant temperature, while Exercise I-4 requires the time-dependent solution of the same cell definition combined with a power transient. The figures of merit for both exercises are the variation in the unit cell temperature profiles due to input uncertainty variations in the material properties and boundary conditions.

This section presents the results of Computational Fluid Dynamics (CFD) study performed with the commercial CFX-13 code $^{25}$ by Dr. Su-Jong Yoon. It was jointly supported by the National Research Foundation of Korea (NRF) and a grant funded by the Korean government (Grant code: 2012-052255). A summary of this information has also been presented at the International Congress on Advances in Nuclear Power Plants (ICAPP) 2014 conference. ${ }^{26}$ It should also be noted that the results of this exercise, as reported here, were based on Revision 1 of the specifications. Some applied densities and dimensions are slightly different to the latest revision, but the general trends and conclusions are still valid.

\subsection{Overview of Specifications: Exercise I-3}

A typical hexagonal MHTGR-350 unit cell is shown in Figure 42, consisting of a helium coolant channel, six fuel compacts and a matrix graphite region. The helium gaps around the fuel compacts are also included. The equivalent triangular unit cells are shown in Figure 43. The only difference between the two sub-exercises is the representation of the fuel region.

- Exercise I-3a: The fuel region is modeled as a volume-averaged homogenous mixture consisting of $\mathrm{H}-451$ graphite and TRISO fuel kernels.

- Exercise I-3b: The fuel region is modeled as a heterogeneous mixture of TRISO fuel kernels embedded in $\mathrm{H}-451$ graphite (i.e., the UCO, inner and outer pyrolitic carbon [IPyC/OPyC] and silicon carbide [SiC] layers of the TRISO fuel particles are explicitly modeled). 


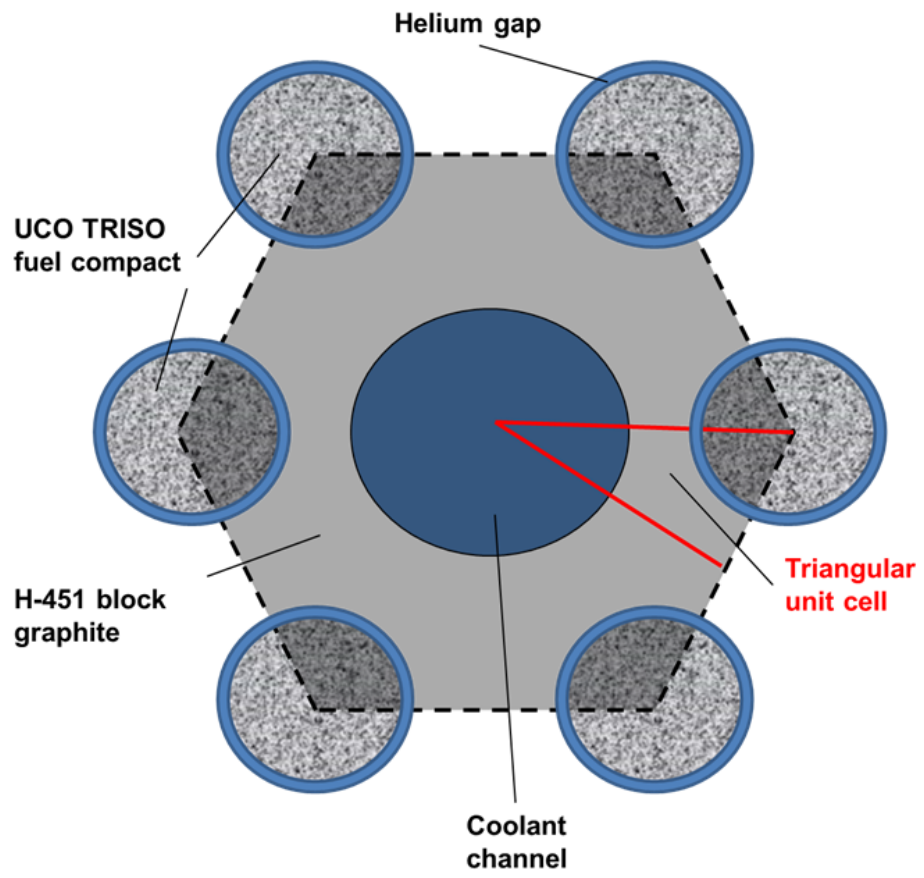

Figure 42. MHTGR-350 hexagonal and triangular unit cell representations.

Ex. I-3a

\section{Compact matrix graphite and} TRISOs (homogeneous)

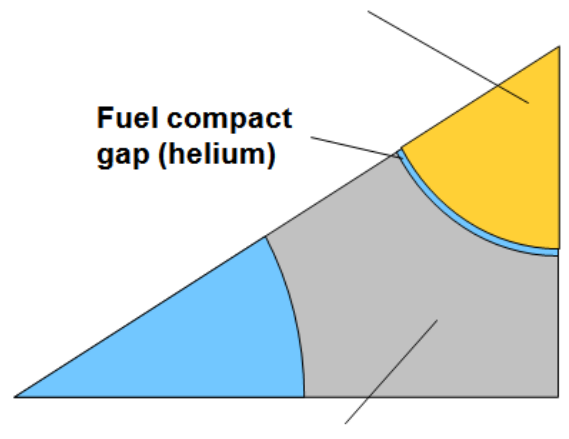

H-451 graphite
Ex. I-3b

Compact matrix graphite and TRISOs (heterogeneous)

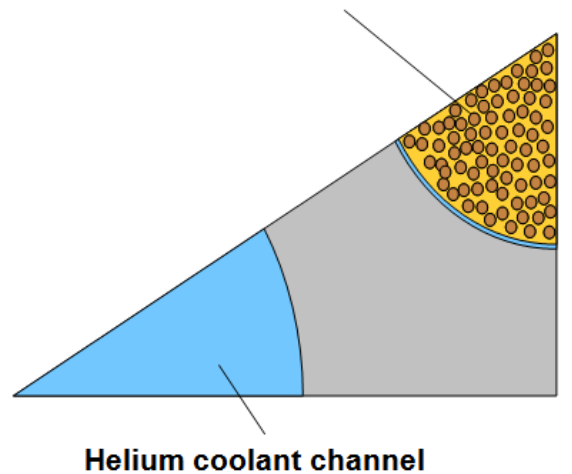

Figure 43. MHTGR-350 triangular unit cells for Exercise I-3a/4a and Exercise I-3b/4b.

The geometry and material properties are based on the OECD/NEA MHTGR-350 MW benchmark specification. ${ }^{5}$ The fuel-lattice unit cell is assumed to be a two-dimensional symmetric $1 / 12$ th model of the MHTGR-350 design, with dimensions as shown in Figure 44. 


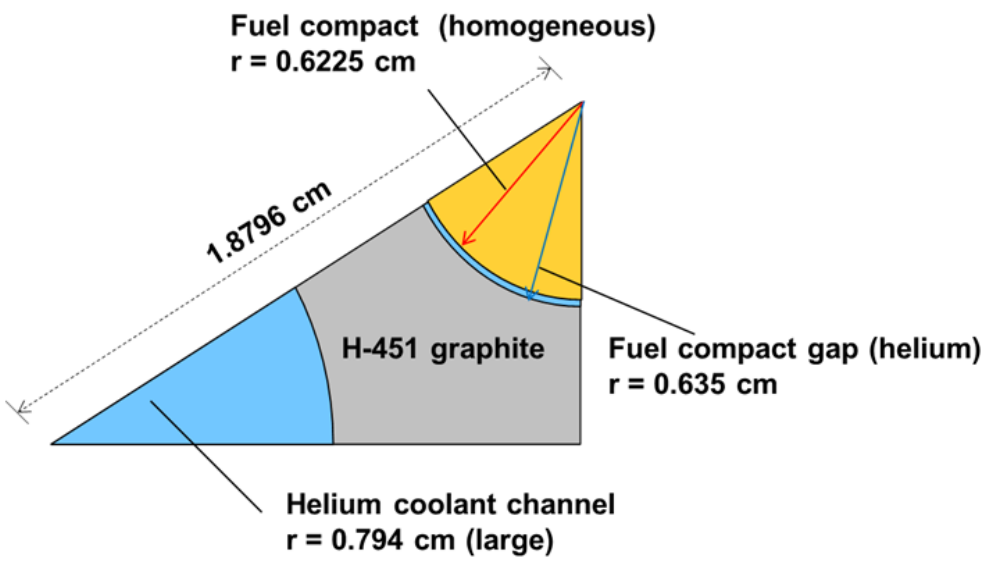

Figure 44. MHTGR-350 triangular unit cell specification.

The following assumptions and input boundary conditions are made:

- There are 102 large and six small coolant holes per standard fuel element. For this exercise, only the large coolant hole geometry will be investigated (i.e., $r=0.794 \mathrm{~cm}$ ).

- The coolant channel is not implicitly included in the unit cell. This is instead modeled as a simple heat sink boundary with a bulk coolant temperature of $750 \mathrm{~K}$ and a constant heat transfer coefficient of $1,700 \mathrm{~W} / \mathrm{m}^{2} \mathrm{~K}$.

- The heat transfer coefficient value $\left(1,700 \mathrm{~W} / \mathrm{m}^{2} \cdot \mathrm{K}\right)$ is calculated based on nominal MHTGR-350 operating conditions at a pressure of $6.39 \mathrm{MPa}$ using standard pipe flow correlations. The Petukhov correlation ${ }^{27}$ has been used to calculate the Darcy friction factor and the Gnielinski correlation ${ }^{28}$ has been used to calculate the Nusselt number at a Reynolds number of 43,000.

- The remaining boundaries of the model are assumed adiabatic (i.e., heat conduction to adjacent unit cells is ignored).

- A fixed power density in the fuel compact of $26 \mathrm{MW} / \mathrm{m}^{3}$ is used. This is based on the nominal MHTGR operating power of $350 \mathrm{MW}$ assuming a uniform power distribution. A schematic representation of these nominal case values are shown in Figure 45.

- Heat transfer across the $0.125 \mathrm{~mm}$ gap between the fuel compact and H-451 graphite region is modeled assuming heat conduction through stagnant helium and radiative heat transfer using an emissivity value of 0.85 for $\mathrm{H} 451$ graphite.

- The complex dependence of the thermal conductivity on temperature and fluence requires specific attention. The same correlations prescribed for the OECD/NEA MHTGR-350 benchmark $^{5}$ are used in this CRP specification. 
$T_{\mathrm{He}}=750 \mathrm{~K}$ $h=1700 \mathrm{~W} / \mathrm{m}^{2} . \mathrm{K}$

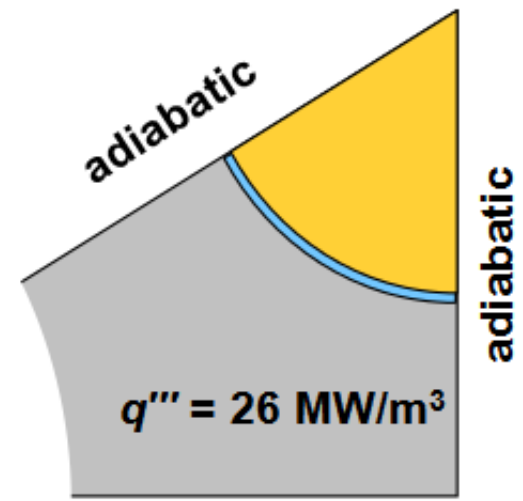

adiabatic

Figure 45. MHTGR-350 unit cell boundary conditions.

The nominal (i.e., best estimate, expected or mean) values used as input for Exercise I-3 and I-4 are included in Table 21.

Table 21. Exercise I-3 and I-4 input parameters - nominal values.

\begin{tabular}{|l|l|}
\hline \multicolumn{1}{|c|}{ Input Parameter } & \multicolumn{1}{c|}{ Nominal/Mean Value } \\
\hline Fuel compact power density & $26 \mathrm{MW} / \mathrm{m}^{3}$ \\
\hline Helium coolant temperature & $750 \mathrm{~K}$ \\
\hline Heat transfer coefficient & $1,700 \mathrm{~W} / \mathrm{m}^{2} . \mathrm{K}$ \\
\hline TRISO packing fraction & 0.35 \\
\hline Fuel compact/graphite gap width & $0.125 \mathrm{~mm}$ \\
\hline Thermal conductivities & Correlations (Table 24-Table 27) \\
\hline Specific heat (includes density as $\left.\rho c_{\mathrm{p}}\right)$ & Correlations (Table 24-Table 27) \\
\hline Emissivity & 0.85 \\
\hline UCO kernel diameter $(\mu \mathrm{m})$ & 349.7 \\
\hline UCO kernel density $(\mathrm{kg} / \mathrm{m} 3)$ & $1,092.4$ \\
\hline Buffer thickness $(\mu \mathrm{m})$ & 103.5 \\
\hline IPyC thickness $(\mu \mathrm{m})$ & 39.4 \\
\hline SiC thickness $(\mu \mathrm{m})$ & 35.3 \\
\hline OPyC thickness $(\mu \mathrm{m})$ & 41.0 \\
\hline Buffer density $\left(\mathrm{kg} / \mathrm{m}^{3}\right)$ & 970 \\
\hline IPyC density $\left(\mathrm{kg} / \mathrm{m}^{3}\right)$ & 1,900 \\
\hline SiC density $\left(\mathrm{kg} / \mathrm{m}^{3}\right)$ & 4,210 \\
\hline OPyC density $\left(\mathrm{kg} / \mathrm{m}^{3}\right)$ & 1,900 \\
\hline
\end{tabular}


Table 22. Thermo-physical properties of H-451 graphite.

\begin{tabular}{|l|l|}
\hline \multicolumn{1}{|c|}{ Parameter } & \multicolumn{1}{c|}{ Value } \\
\hline Thermal conductivity $[\mathrm{W} / \mathrm{m} / \mathrm{K}]$ & $\mathrm{k}=4.19346^{*} 10^{-6} \mathrm{~T}^{2}-2.13523 * 10^{-2} \mathrm{~T}+5.41993 * 10$ \\
\hline Density $\left[\mathrm{kg} / \mathrm{m}^{3}\right]$ & 1,740 \\
\hline Specific Heat $[\mathrm{J} / \mathrm{kg} / \mathrm{K}]$ & $\mathrm{c}_{\mathrm{p}}=4,184^{*}\left(0.54212-2.42667 * 10^{-6} \mathrm{~T}-90.2725 \mathrm{~T}^{-1}-\right.$ \\
& $\left.43,449.3 \mathrm{~T}^{-2}+1.59309 * 10^{7} \mathrm{~T}^{-3}-1.43688^{*} 10^{9} \mathrm{~T}^{-4}\right)$ \\
\hline Emissivity & 0.85 \\
\hline
\end{tabular}

Table 23. Thermo-physical properties of pyrolytic carbon layer.

\begin{tabular}{|l|l|}
\hline \multicolumn{1}{|c|}{ Parameter } & \multicolumn{1}{c|}{ Value } \\
\hline Thermal conductivity $[\mathrm{W} / \mathrm{m} / \mathrm{K}]$ & $\begin{array}{l}\mathrm{k}_{\mathrm{PyC}}=244.3 \mathrm{~T}^{-0.574}\left[1-0.3662\left(1-\mathrm{e}^{-1.005 \Gamma}\right.\right. \\
-0.03554 \Gamma)]^{*}\left[\rho_{\mathrm{PyC}} /\left(2.2\left(1,930-\rho_{\mathrm{PyC}}\right)+\rho_{\mathrm{PyC}}\right)\right] \\
\end{array}$ \\
& $\begin{array}{l}\mathrm{k}_{\mathrm{PC}}=122.15 \mathrm{~T}^{-0.574}\left[1-0.3662\left(1-\mathrm{e}^{-1.005 \Gamma}\right.\right. \\
-0.03554 \Gamma)]^{*}\left[\rho_{\mathrm{PC}} /\left(2.2\left(1,930-\rho_{\mathrm{PC}}\right)+\rho_{\mathrm{PC}}\right)\right]\end{array}$ \\
\hline Density PyC $\left[\mathrm{kg} / \mathrm{m}^{3}\right]$ & 1,900 \\
\hline Density PC $\left[\mathrm{kg} / \mathrm{m}^{3}\right]$ & 970 \\
\hline Specific Heat $[\mathrm{J} / \mathrm{kg} / \mathrm{K}]$ & $\begin{array}{l}\mathrm{c}_{\mathrm{p}}=4,184 *\left(0.54212-2.42667 * 10^{-6} \mathrm{~T}-90.2725 \mathrm{~T}^{-1}-43,449.3 \mathrm{~T}^{-2}\right. \\
\left.+1.59309^{*} 10^{7} \mathrm{~T}^{-3}-1.43688^{*} 10^{9} \mathrm{~T}^{-4}\right)\end{array}$ \\
\hline
\end{tabular}

Table 24. Thermo-physical properties of silicon carbon layer.

\begin{tabular}{|l|l|}
\hline \multicolumn{1}{|c|}{ Parameter } & \multicolumn{1}{c|}{ Value } \\
\hline Thermal conductivity $[\mathrm{W} / \mathrm{m} / \mathrm{K}]$ & $\mathrm{k}=(17,885 / \mathrm{T}+2) \mathrm{e}^{-0.1277 \Gamma}$ \\
\hline Density PyC $\left[\mathrm{kg} / \mathrm{m}^{3}\right]$ & 4,210 \\
\hline Specific Heat $[\mathrm{J} / \mathrm{kg} / \mathrm{K}]$ & $\mathrm{c}_{\mathrm{p}}=925.65+0.3772 \mathrm{~T}-7.9259 * 10^{-5} \mathrm{~T}^{2}$ \\
& $-3.1946^{*} 10^{7} \mathrm{~T}^{-2}$ \\
\hline
\end{tabular}

Table 25. Effective thermo-physical properties of fuel compact.

\begin{tabular}{|l|l|}
\hline \multicolumn{1}{|c|}{ Parameter } & \multicolumn{1}{c|}{ Value } \\
\hline Thermal conductivity $[\mathrm{W} / \mathrm{m} / \mathrm{K}]$ & $\begin{array}{l}\mathrm{k}=47.4 *\left(1-9.755610^{-4}(\mathrm{~T}-373.15) \mathrm{e}^{-6.03610-4(\mathrm{~T}-273.15)}\right)^{*}[1-0.3662(1- \\
\left.\mathrm{e}^{-1.005 \Gamma}-0.03554 \Gamma\right) *[\rho /(2.2(1,700-\rho)+\rho)]\end{array}$ \\
\hline Density $\left[\mathrm{kg} / \mathrm{m}^{3}\right]$ & 1,740 \\
\hline Specific Heat $[\mathrm{J} / \mathrm{kg} / \mathrm{K}]$ & $\begin{array}{l}\mathrm{c}_{\mathrm{p}}=4,184 *\left(0.54212-2.42667 * 10^{-6} \mathrm{~T}\right. \\
\left.-90.2725 \mathrm{~T}^{-1}-43,449.3 \mathrm{~T}^{-2}+1.59309 * 10^{7} \mathrm{~T}^{-3}-1.43688^{*} 10^{9} \mathrm{~T}^{-4}\right)\end{array}$ \\
\hline
\end{tabular}

Since no data are available for the material property of $\mathrm{UC}_{0.5} \mathrm{O}_{1.5}$ kernel in a heterogeneous model, uranium dioxide $\left(\mathrm{UO}_{2}\right)$ properties are used instead. The density of $\mathrm{UO}_{2}$ is specified as $10,970 \mathrm{~kg} / \mathrm{m}^{3}$ in this simulation. The dimensional changes in the kernel density with temperature are not taken into account. The value of the burn-up (B) of kernel is assumed to be 3.415. The model for irradiated $\mathrm{UCO}_{2}$ thermal conductivity is specified in accordance with $^{5}$ as:

$\mathrm{k}[\mathrm{W} / \mathrm{m} / \mathrm{k}]=\mathrm{k}_{0}(\mathrm{~T}) * \mathrm{FD}^{*} \mathrm{FP} * \mathrm{FR}$ 
where $\mathrm{k}_{0}(\mathrm{~T})$ is the thermal conductivity of $100 \%$ dense $\mathrm{UCO}_{2}, \mathrm{FD}$ is a dissolved solid fission product factor, FP is a precipitated solid fission product factor, and FR is a radiation damage factor. The respective factors are determined as follows:

$$
\begin{aligned}
& \mathrm{k}_{0}(\mathrm{~T})=115.8 /\left(7.5408+17.692 \mathrm{t}+3.6142 \mathrm{t}^{2}\right)+7,410.5 \mathrm{t}^{-5} / 2 \mathrm{e}^{-16.35 / \mathrm{t}} \\
& \mathrm{FD}=\left(1.09 / \mathrm{B}^{3.265}+0.0643(\mathrm{~T} / \mathrm{B})^{1 / 2}\right)^{*} \arctan \left[\left(1.09 / \mathrm{B}^{3.265}+0.0643(\mathrm{~T} / \mathrm{B})^{1 / 2}\right)^{-1}\right] \\
& \mathrm{FP}=1+0.019 \mathrm{~B} /(3-0.019 \mathrm{~B})^{*}\left[1+\mathrm{e}^{-(\mathrm{T}-1,200) / 100}\right]^{-1} \\
& \mathrm{FR}=1-0.2 /\left(1+\mathrm{e}^{(\mathrm{T}-900) / 80}\right)
\end{aligned}
$$

where $\mathrm{t}$ is $\mathrm{T}(\mathrm{K}) / 1,000$.

The specific heat capacity model covers the temperature range from $298.15 \mathrm{~K}$ to $3,120 \mathrm{~K}$ and it is functionalized as:

$$
\begin{aligned}
& \mathrm{c}_{\mathrm{p}}=302.27(548.68 / \mathrm{T})^{2} \mathrm{e}^{548.68 / \mathrm{T}} /\left(\mathrm{e}^{548.68 / \mathrm{T}}-1\right)^{2}+2 * 8.463 * 10^{-3}(1+0.011 \mathrm{~B}) \mathrm{T}+8.741 * 10^{7 *} 18,531.7 * \\
& \mathrm{e}^{-18,531.7 / \mathrm{T}} / \mathrm{T}^{2}
\end{aligned}
$$

\subsection{CFX Fuel Models}

In this study CFX $-13^{25}$ was used to obtain the temperature profile in the unit-cell model of MHTGR-350 fuel. Figure 46 shows the cross-sectional view of the fuel compact. In this figure, the sizes of TRISO particles on the cross section are not identical. However, the TRISO particles are assumed to be identical in this analysis. Because the surface area of the TRISO particles on the cross section varies with their locations, the average size of the TRISO particles are determined based on the average packing fraction of the particles in the fuel compact. To compare the homogeneous and heterogeneous fuel models, two-dimensional CFD fuel models are developed as shown in Figure 47 and Figure 48. The height of CFD model $\left(\mathrm{h}_{\mathrm{m}}\right)$ is assumed to be $1 \mathrm{~mm}$ (i.e., a very thin layer that essentially reduces to a two-dimensional approach). The number of TRISO particles in the heterogeneous 2-D model is calculated based on the packing fraction of the fuel compact. The total number of kernels in the fuel compact is calculated as:

$\mathrm{N}_{\text {kernel }}=0.35 \mathrm{~V}_{\text {fc }} / \mathrm{V}_{\text {kernel }} \approx 6,646$

(This number is slightly larger than the number of kernels used in Exercise I-1b, due to the assumptions made in each of the cases. This difference is acceptable since the exercises are not linked in terms of uncertainty propagation. For the coupled calculation that will be defined for Phases II-IV, the neutronic specification will be used consistently). To calculate the number of kernels in the 2D heterogeneous model, the number of kernels per cross section of fuel compact is calculated by dividing the height of the fuel compact into the total number of kernels:

$\mathrm{N}_{\text {kernel,m }}=\left(\mathrm{N}_{\text {kernel }} \mathrm{h}_{\mathrm{m}} / \mathrm{h}_{\mathrm{fc}, \mathrm{m}}\right) / 6 \approx 22$

Since only $1 / 6$ section of the fuel compact is modeled in the unit cell, a total of 22 TRISO fuel particles are explicitly modeled in the heterogeneous model. The TRISO particles in the fuel compact are randomly distributed. To calculate the power density of kernel in the heterogeneous model, the power per fuel compact of the 2-D CFD model is calculated by dividing the fuel compact volume into the fuel compact power density:

$\mathrm{Q}_{\mathrm{fc}, \mathrm{m}}=\mathrm{Q}^{\prime \prime \prime}{ }_{\mathrm{fc}, \mathrm{m}} \mathrm{V}_{\mathrm{fc}, \mathrm{m}}=\mathrm{Q}^{\prime \prime \prime}{ }_{\mathrm{fc}, \mathrm{m}}\left(\mathrm{A}_{\mathrm{fc}, \mathrm{m}} \mathrm{h}_{\mathrm{m}}\right)=0.527 \mathrm{~W}$

The power per kernel can then be determined by dividing the number of kernels into the power per fuel compact:

$\mathrm{Q}_{\mathrm{kernel}, \mathrm{m}}=\mathrm{Q}_{\mathrm{fc}, \mathrm{m}} / \mathrm{N}_{\mathrm{kernel}, \mathrm{m}} \approx 0.024 \mathrm{~W}$ 
Finally, the power density of a single kernel is calculated by dividing the volume of the kernel into the power per kernel:

$\mathrm{Q}^{\prime \prime \prime}{ }_{\text {kernel, } \mathrm{m}}=\mathrm{Q}_{\text {kernel,m }} / \mathrm{V}_{\text {kernel, } \mathrm{m}} \approx 169 \mathrm{MW} / \mathrm{m}^{3}$

A hexahedral mesh is used for the homogeneous model (Figure 47) while the hybrid mesh combining the hexahedral and wedge meshes is used in the heterogeneous model (Figure 48).

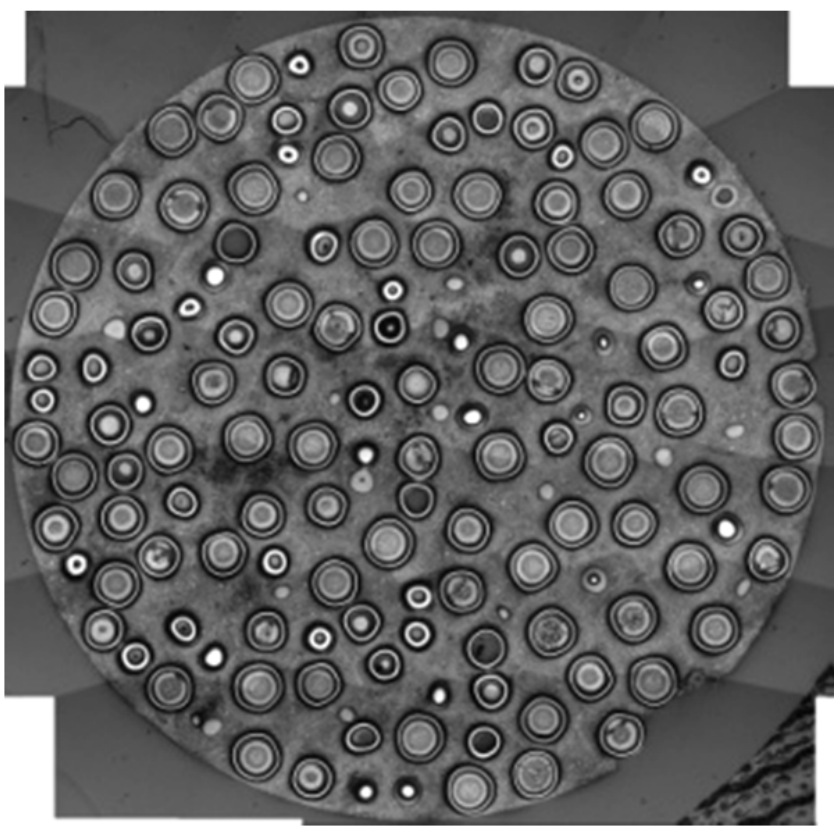

Figure 46. Cross-sectional view of a fuel compact (micrograph). The varying radii of the particles indicate the variable location of the randomly distributed particles relative to the cut-plane.

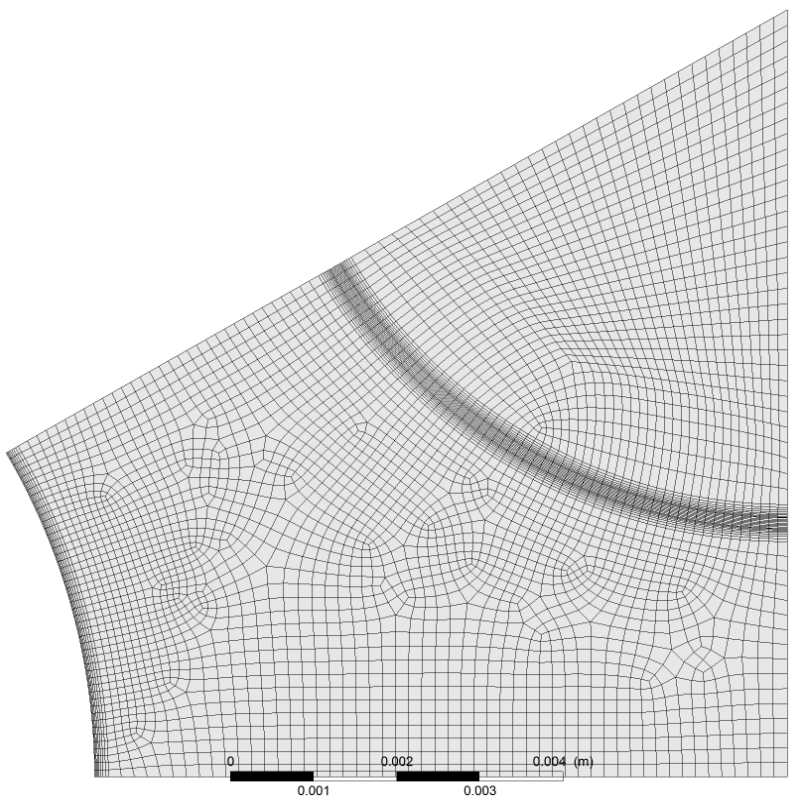

Figure 47. Homogeneous unit-cell model of MHTGR-350 fuel. 


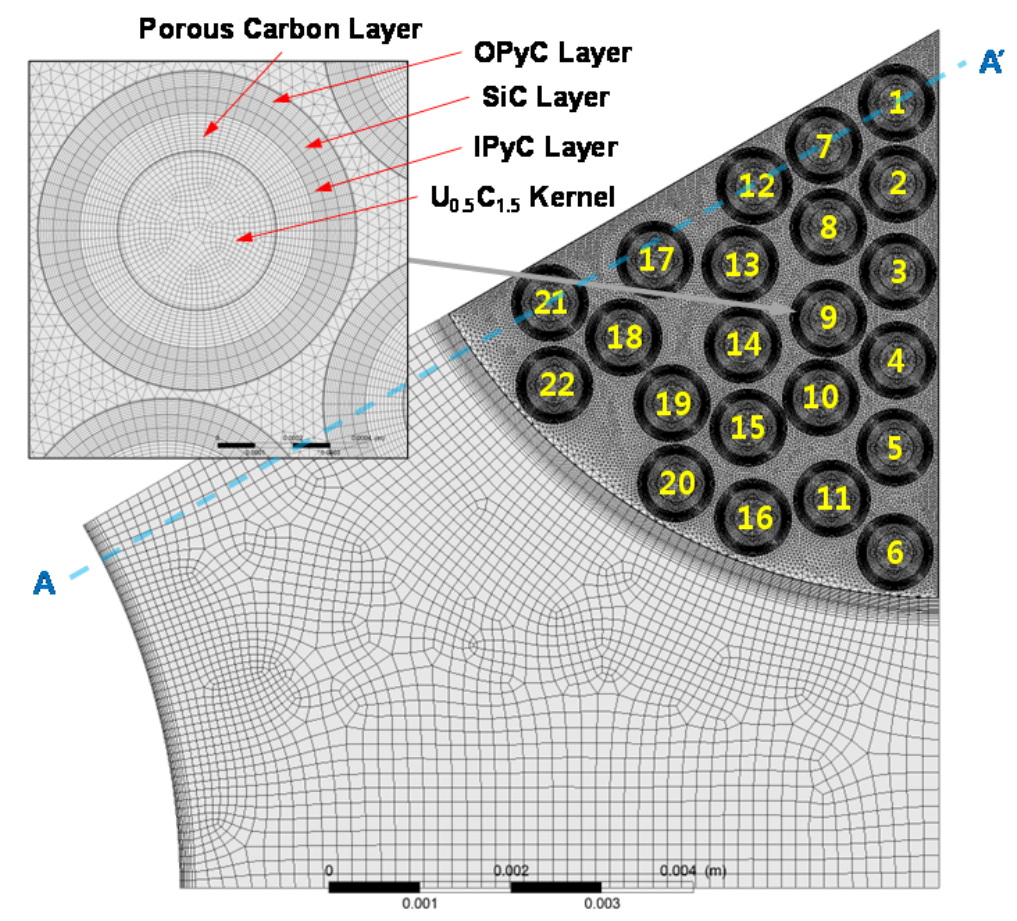

Figure 48. Heterogeneous unit-cell model of MHTGR-350 fuel.

For the graphite and fuel gap regions, the same mesh structure was used for both the homogeneous and heterogeneous models. The mesh information is summarized in Table 26.

Table 26. Mesh Statistics of CFD Models.

\begin{tabular}{|l|c|c|}
\hline \multicolumn{1}{|c|}{ Parameter } & Homogeneous & Heterogeneous \\
\hline Number of Nodes & 10,682 & 185,390 \\
\hline Number of Elements & 5,080 & 90,207 \\
\hline Wedges & 0 & 13,466 \\
Hexahedral & 5,080 & 76,741 \\
\hline
\end{tabular}

The maximum residual value of $10^{-9}$ was used to monitor the convergence of the simulation. The Discrete Transfer Model is employed to solve the radiation heat transfer through the gap between the graphite and fuel compact. In the transient simulation, the time step and total simulation time are $0.1 \mathrm{sec}$ and $30 \mathrm{sec}$, respectively. The input parameters, boundary conditions and material properties discussed in the Section 7.1 are adopted as the inputs of the CFD simulations.

\subsection{Results and Discussion}

\subsubsection{Exercise I-3: Steady-State Results}

The temperature distributions of the homogeneous (Exercise I-3a) and heterogeneous (Exercise I-3b) models are shown in Figure 49 and Figure 50, respectively. In the steady-state simulation, the maximum temperature of the homogeneous model was observed at the center of the fuel compact because this location is farthest away from the heat sink. 
The maximum temperature of heterogeneous model was observed in the $\mathrm{U}_{0.5} \mathrm{C}_{1.5}$ kernel of first TRISO particle, which is also farthest away from the heat sink. The maximum and volume-averaged temperatures of fuel compact in the homogeneous model and heterogeneous model are $883 \mathrm{~K}$ and $899 \mathrm{~K}$, respectively.

The temperatures of different structures of the compact as computed for the homogeneous and heterogeneous models are summarized in Table 27 and Table 28, respectively. In these analyses, the temperatures of fuel gap and graphite in the homogeneous and heterogeneous models are similar to each other because total heat generation in the fuel compact of the homogeneous and heterogeneous model is preserved. In the heterogeneous model, the maximum and volume-averaged temperatures of the kernels were similar to each other. Thus, the temperature deviation inside the kernels is negligible. A variation up to $24 \mathrm{~K}$ is observed between $22 \mathrm{kernels}$, depending on their spatial locations. However, the graphite region in the heterogeneous fuel compact shows a larger difference between the volume-averaged and maximum temperatures.

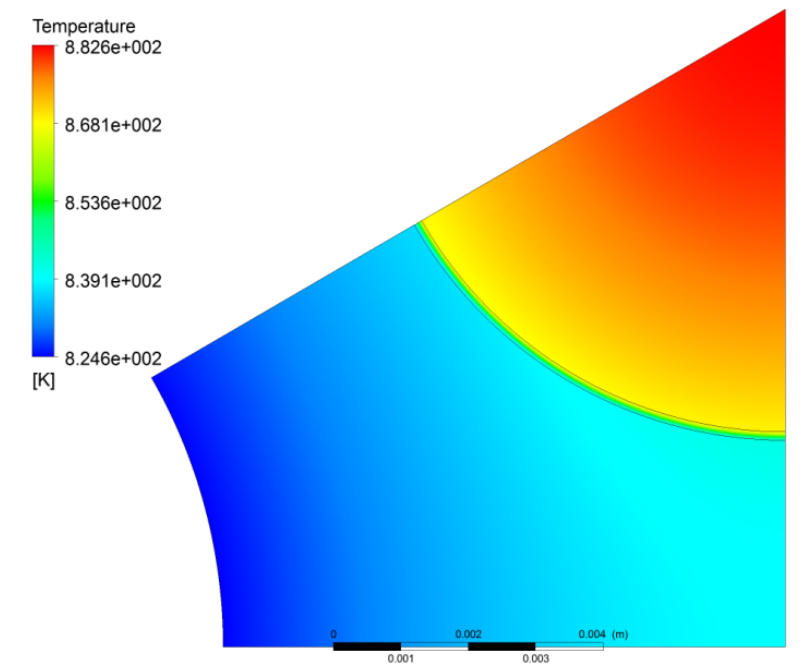

Figure 49. Temperature (K) distribution of the steady-state homogeneous model (Exercise I-3a).

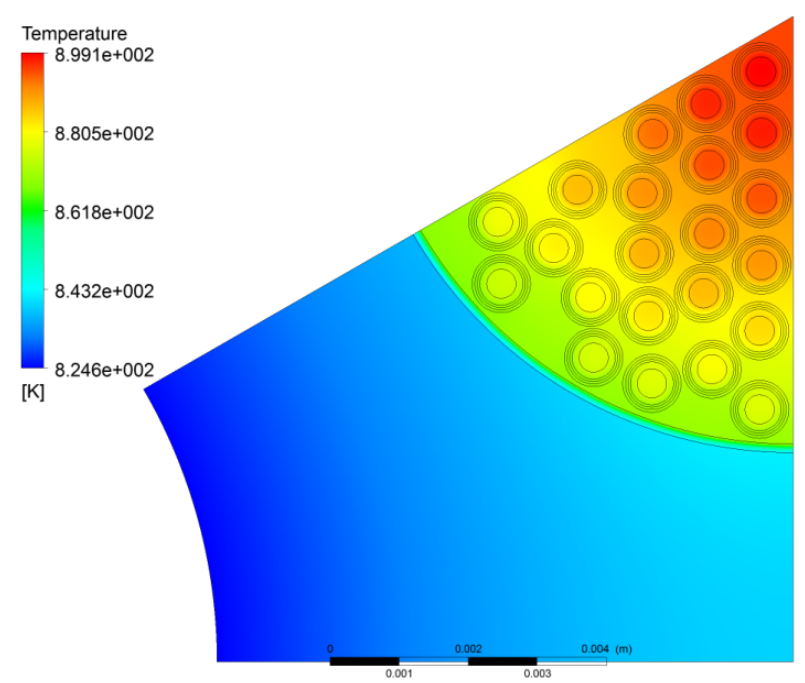

Figure 50. Temperature (K) distribution of the steady-state heterogeneous model (Exercise I-3b). 
Table 27. Volume-averaged and maximum temperature $(\mathrm{K})$ of the homogeneous model.

\begin{tabular}{|l|c|c|}
\hline \multicolumn{1}{|c|}{ Location } & Volume-averaged & Maximum \\
\hline Fuel Compact (mixture) & 875.6 & 882.6 \\
\hline Fuel Gap & 853.8 & 869.2 \\
\hline Graphite & 833.9 & 841.6 \\
\hline
\end{tabular}

Table 28. Volume-averaged and maximum temperature $(\mathrm{K})$ of the heterogeneous model.

\begin{tabular}{|l|c|c|}
\hline \multicolumn{1}{|c|}{ Location } & Volume-averaged & Maximum \\
\hline Fuel Compact (graphite) & 879.4 & 895.2 \\
\hline Fuel Gap & 853.8 & 869.7 \\
\hline Graphite & 833.9 & 841.6 \\
\hline Kernel 1 & 898.8 & 899.1 \\
\hline Kernel 2 & 897.4 & 897.7 \\
\hline Kernel 3 & 894.2 & 894.6 \\
\hline Kernel 4 & 889.5 & 890.0 \\
\hline Kernel 5 & 883.6 & 884.1 \\
\hline Kernel 6 & 876.2 & 876.8 \\
\hline Kernel 7 & 896.8 & 897.1 \\
\hline Kernel 8 & 894.5 & 894.9 \\
\hline Kernel 9 & 891.1 & 891.4 \\
\hline Kernel 10 & 886.3 & 886.8 \\
\hline Kernel 11 & 879.2 & 879.8 \\
\hline Kernel 12 & 892.5 & 893.0 \\
\hline Kernel 13 & 889.8 & 890.2 \\
\hline Kernel 14 & 887.3 & 887.7 \\
\hline Kernel 15 & 882.7 & 883.3 \\
\hline Kernel 16 & 876.1 & 876.7 \\
\hline Kernel 17 & 886.2 & 886.6 \\
\hline Kernel 18 & 881.4 & 882.1 \\
\hline Kernel 19 & 880.2 & 881.0 \\
\hline Kernel 20 & 875.4 & 876.0 \\
\hline Kernel 21 & 878.3 & 874.8 \\
\hline Kernel 22 & 874.3 & \\
\hline & & \\
\hline & & \\
\hline
\end{tabular}

A comparison of the temperature profile between the homogeneous and heterogeneous models is shown in Figure 51. For both models, temperature profiles in the graphite and fuel gap regions were identical while quite different profiles in the fuel compact region were observed. Generally, the temperatures of the TRISO particles in the heterogeneous model were higher than those at the same location in the homogeneous model. Thus, this under-estimation by the homogeneous model for predicting the peak fuel temperatures should be considered in reactor safety analyses that assume a homogeneous model. 
In this analysis, the uniform fuel gap assumes complete separation of the fuel compact and graphite region. The heat is transferred by conduction and radiation in the gap region. However, in the actual situation, this fuel gap would not be uniform, and contact heat transfer between the fuel compact and graphite could occur. Due to the change of heat transfer mechanism, the temperature of fuel compact and graphite could change. This aspect would need further investigation in the case of realistic reactor safety analyses. However, for this uncertainty calculation, uniform heat transfer across the gap is assumed.

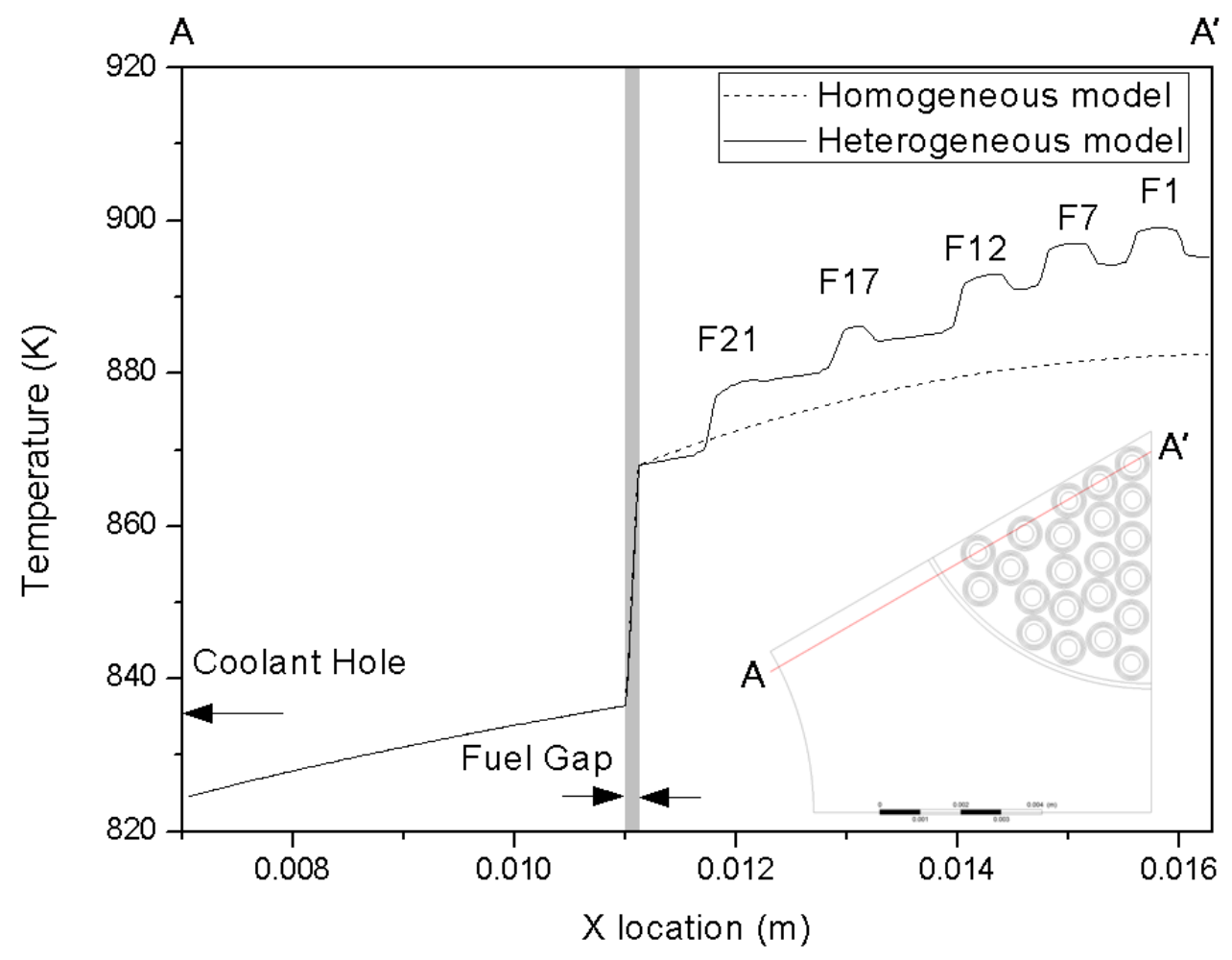

Figure 51. Comparison of temperature $(\mathrm{K})$ profiles between homogeneous and heterogeneous models.

\subsubsection{Exercise I-4: Transient Results}

The transient simulation used the converged steady-state simulation as an initial condition. Figure 52 shows the maximum and volume-averaged temperatures of the homogeneous model (Exercise I-4a) in the transient simulation. The temperature of the fuel compact reached the peak value after 6.4 seconds from the point transient power reach peak. The peak temperatures in the fuel compact, fuel gap, and graphite were $1,079 \mathrm{~K}, 1,018 \mathrm{~K}$, and $942 \mathrm{~K}$, respectively. The peak volume-averaged temperatures of the fuel compact, fuel gap and graphite were 1,047 K, $971 \mathrm{~K}$, and $925 \mathrm{~K}$, respectively. Although the transient power increased to 10 times higher than the nominal power, the maximum temperature of fuel compact did not exceed $1,100 \mathrm{~K}$. 


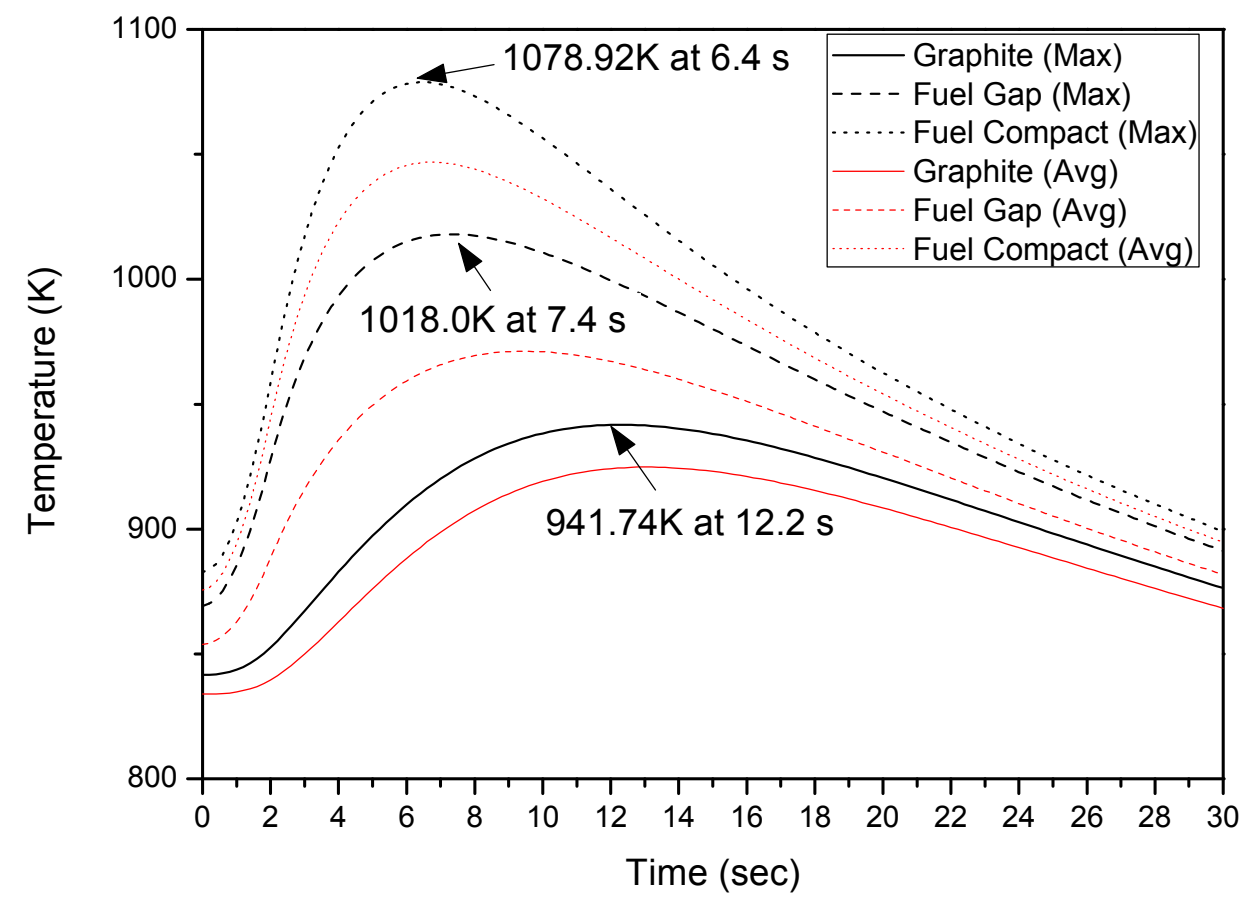

Figure 52. Maximum and volume-averaged temperatures (K) of homogeneous model in a transient simulation.

Figure 53 and Figure 54 show the maximum and volume-averaged temperatures of heterogeneous model in the transient simulation (Exercise I-4b), respectively. The peak temperatures of first kernel, fuel compact, fuel gap, and graphite were $1,138 \mathrm{~K}, 1,120 \mathrm{~K}, 1,013 \mathrm{~K}$, and $938 \mathrm{~K}$, respectively.

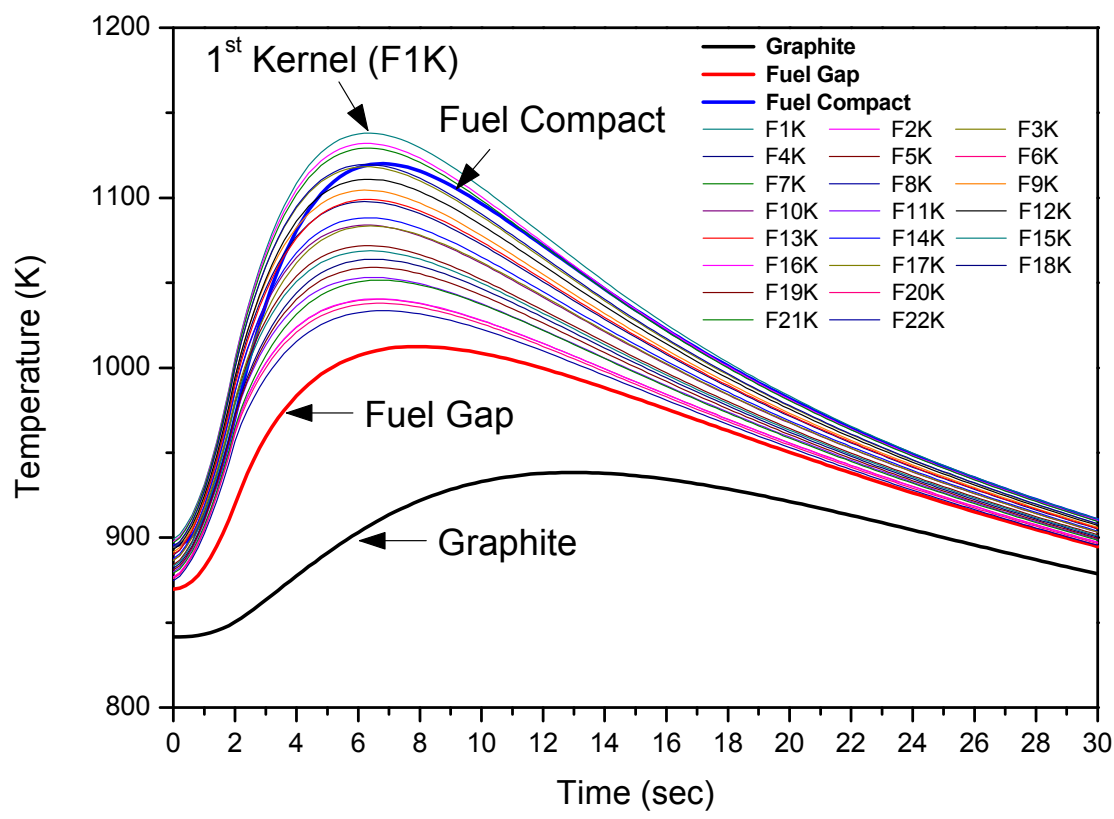

Figure 53. The maximum temperature $(\mathrm{K})$ profile of heterogeneous model in a transient simulation. 


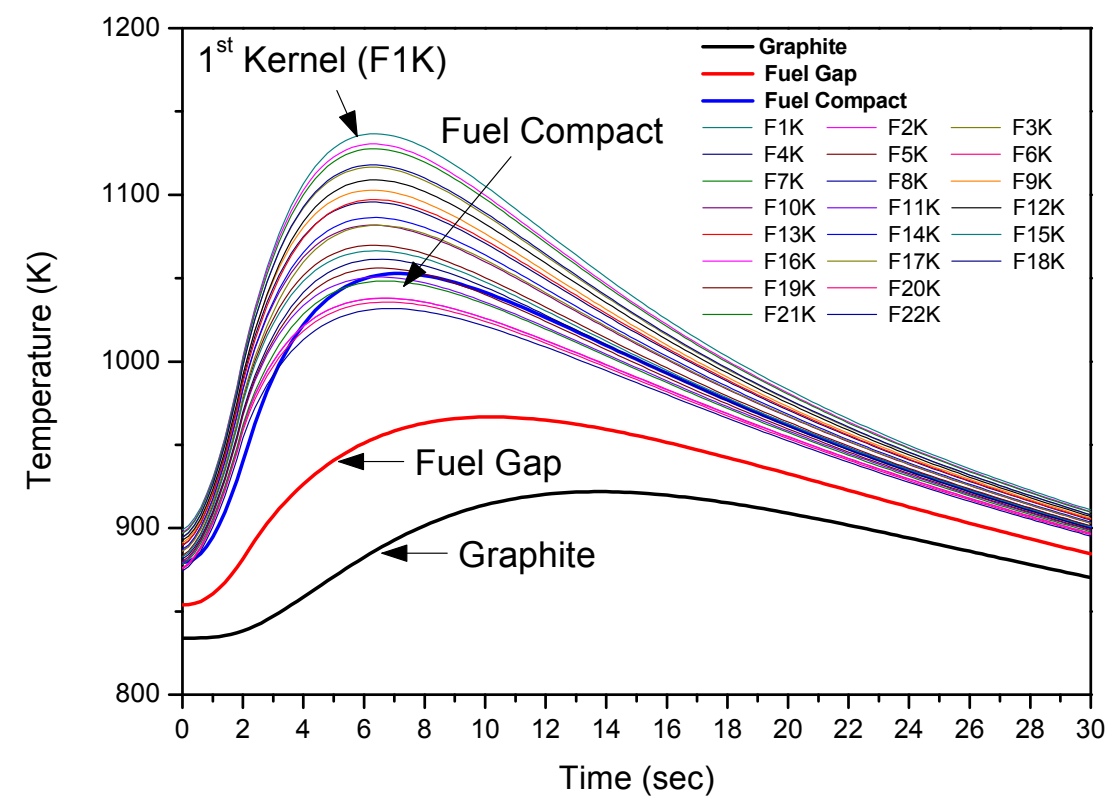

Figure 54. The volume-averaged temperature $(\mathrm{K})$ profile of heterogeneous model in a transient simulation.

A comparison of maximum temperatures of homogeneous and heterogeneous models in the transient simulation is presented in Figure 55. Similarly to the results of steady-state simulation shown in Figure 51, the temperatures of the fuel gap and graphite in homogeneous and heterogeneous models were close to each other. The temperature inside the fuel compact of heterogeneous model, in contrast, was significantly higher than that of homogeneous model. Assuming the heterogeneous model captures the relevant physics more completely and accurately, the homogeneous model for the fuel compact of prismatic HTGR tends to underestimate the peak fuel temperature by up to $10 \%$. However, this statement can only be qualified by performing validation comparisons against experimental or operational data.

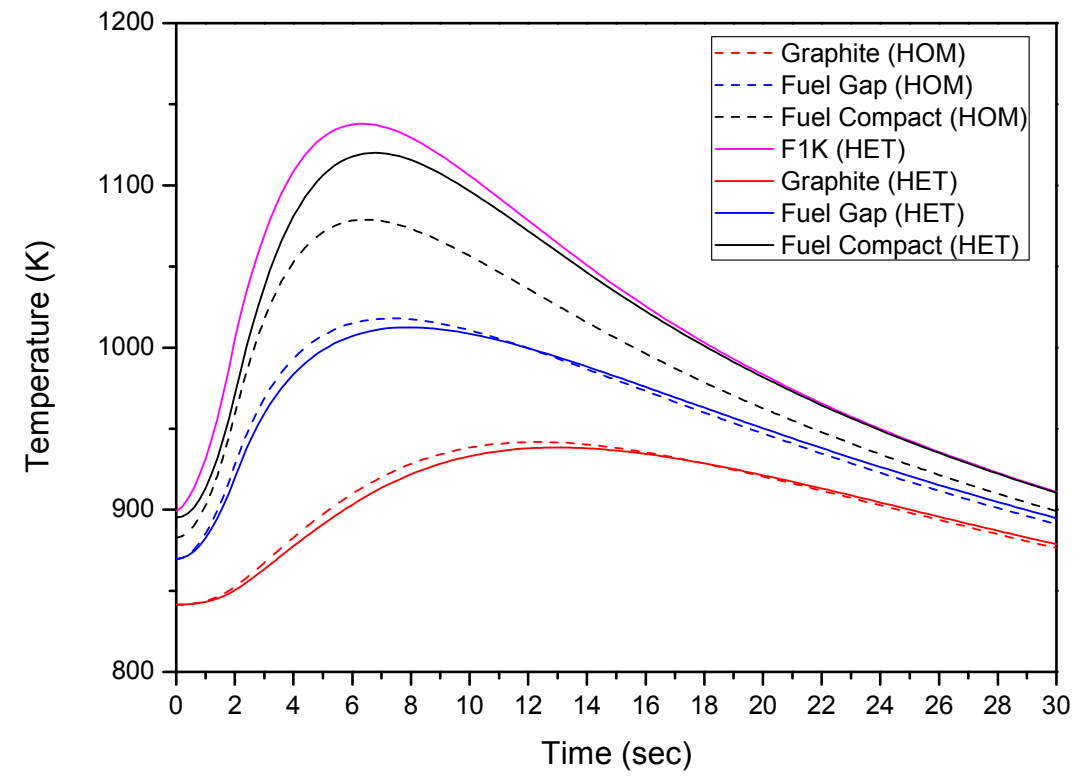

Figure 55. Comparison of maximum temperatures between the homogeneous and heterogeneous model in the transient simulation. 


\subsection{Summary: Exercise $\mathrm{I}-3$ and $\mathrm{I}-4$}

CFD analyses have been performed for Exercises I-3 and I-4 of the IAEA CRP on HTR UAM to investigate the effect of fuel compact homogenization on the fuel and graphite temperatures, and provide high-fidelity reference results for comparison with lower-order code predictions. The volume-averaged and maximum temperatures of the homogeneous and heterogeneous models were compared for both steady-state and transient conditions. In both steady-state and transient conditions, the peak temperature of the heterogeneous model was significantly higher (up to 10\%) than that of the homogeneous model. The use of homogeneous models in the reactor safety analysis could be non-conservative because this model underestimates the peak temperature of the fuel.

\section{SUMMARY AND FUTURE WORK}

This report presents the status of the INL contributions to Phase I of the IAEA CRP on HTGR uncertainties, as of the date of this report revision. The main focus of the report is the compilation and discussion of reference results for Phase I (i.e., for input parameters at their nominal or best-estimate values), which is defined as the first step of the uncertainty quantification process.

For the validation exercise of this phase, the cell and lattice phases are represented by the VHTRC. The simulations were performed with the Monte Carlo codes SCALE/KENO-VI and Serpent 2. Unit cell simulations of the VHTRC were also performed with MCNP5. In addition to simulations using the ENDF-B-VII.0 cross-section library, the ENDF-B-VII.1 data library was applied for several simulations. KENO-VI simulations were performed using both $\mathrm{CE}$ and $\mathrm{MG}$ cross sections. In case of MG calculations, the DOUBLEHET cell treatment was applied.

A comparison with a subset of the current literature did not show a consistent calculation bias using a regular particle lattice in comparison to a random distribution. The deviation of the multiplication factor of lattice calculations seems to be mainly influenced by the particle pitch in the lattice. The results of KENO-CE calculations of the MHTGR and the VHTRC fuel block exceed those of the respective Serpent calculations for both random and regular particle distributions. This trend agrees with published simulations of the HTTR, a prismatic high-temperature reactor with annular fuel compacts.

Due to an increase in the neutron capture cross section of graphite in the ENDF-B-VII.1 cross-section library, Serpent calculations using this dataset revealed significantly decreased multiplication factors compared to the same calculations using ENDF-VII.0 data. Furthermore, Serpent calculations of the VHTRC fuel assembly suggest a temperature influence on the multiplication factor due to the thermal graphite scattering data. VHTRC calculations using ENDF-V-VII.1 data produced the closest results to the experiment. After removal of assumed simplifications in the VHTRC model, good agreement with the Japanese MVP-II calculations using the JENDL-4.0 library was also obtained.

In CE calculations with SCALE 6.1.2, the cross sections are not adjusted according to the requested temperatures, but defaults to the closest temperature point on the library. The deviation to experimental results varies significantly. In contrast, the requested temperatures are calculated in KENO-VI MG by interpolation, but these results still show a consistent and significant (more than 1\%) overestimation of the experimental dataset. Because SCALE does not yet provide the option of using ENDF-B-VII.1 data, the influence of this library on the KENO-VI simulations could not be investigated. An influence similar to the Serpent calculations is expected.

In addition to simulations with SCALE Version 6.1.2, simulations with SCALE 6.2 beta Version 3 were performed. The best agreement with the Serpent reference result was produced using the 252-group DOUBLEHET models of the MHTGR problem. However, this beta version might still be modified before the first official release of SCALE 6.2.

CFD analyses have been performed for Exercises I-3 and I-4 of the CRP to investigate the effect of fuel compact homogenization on fuel and graphite temperatures, and provide high-fidelity reference 
results for comparison with lower-order code predictions. The volume-averaged and maximum temperatures of the homogeneous and heterogeneous models were compared for both steady-state and transient conditions. In both steady-state and transient conditions, the peak temperature of the heterogeneous model was significantly higher (up to 10\%) than the homogeneous model. The use of homogeneous models in the reactor safety analysis could be non-conservative because this model underestimates the peak temperature of the fuel.

As the propagation of uncertainties through the subsequent coupled core stage requires few-group transport solutions, work has already started at INL on using the NEWT transport solver in SCALE to create equivalent cell, block, and super-cell multi-group lattice models, and to compare the results with the Monte Carlo results reported here. The first NEWT results obtained is discussed in Appendix A.

The NEWT module will also be used, as part of the SAMPLER sequence, to produce perturbed multi- and few-group libraries for use in the propagation of uncertainties through Phases I - III. The next INL CRP progress report, scheduled for release in October 2015, will contain a comparison of the NEWT results for all Phase I exercises, as well as a comparison between the Phase I uncertainty data produced by TSUNAMI-MG (SCALE 6.1), TSUNAMI-CE (using the new CLUTCH and IFP methods in SCALE 6.2), and SAMPLER. 


\section{REFERENCES}

1. IAEA, 2003, Evaluation of High Temperature Gas Cooled Reactor Performance: Benchmark Analysis Related to Initial Testing of the HTTR and HTR-10, International Atomic Energy Agency, IAEA-TECDOC-1382, November 2003.

2. F. Reitsma, et al., "The OECD/NEA/NSC PBMR400 MW Coupled Neutronics Thermal Hydraulics Transient Benchmark - Steady-state Results and Status," Proceedings of PHYSOR-2008, Interlaken, Switzerland, September 14, 2008.

3. G. Strydom, et al., "THE OECD/NEA/NSC PBMR 400 MW Coupled Neutronics Thermal Hydraulics Transient Benchmark: Transient Results," Proceedings of PHYSOR-2010, Pittsburgh, USA, May 9-14, 2010.

4. OECD Nuclear Energy Agency NEA/NSC/DOC, "Benchmark for Uncertainty Analysis in Modelling (UAM) for Design, Operation and Safety Analysis of LWRs, Volume I: Specification and Support Data for the Neutronics Cases (Phase I)," Version 2.0, 2012.

5. OECD Nuclear Energy Agency, 2013, Prismatic Coupled Neutronics/Thermal Fluids Transient Benchmark of the MHTGR-350 MW Core Design: Benchmark Definition, OECD Nuclear Energy Agency NEA/NSC/DOC(2013), 2013.

6. G. Strydom, et al., 2014, HTGR Reactor Physics, Thermal-Hydraulics and Depletion Uncertainty Analysis. Prismatic Benchmark Definition: Phase I, IAEA Draft Rev. 2, November 2014.

7. V. Descotes, et al., "Studies of 2D Reflector Effects in Cross-Section Preparation for Deep Burn VHTRs," Nuclear Engineering and Design, Vol. 242, January 2012, pp. 148-156.

8. J. Leppänen, Development of a new Monte Carlo reactor physics code, Ph.D., Thesis, Helsinki University of Technology, June 18, 2007.

9. ORNL/TM-2005/39, 2011, SCALE: A comprehensive modeling and simulation suite for nuclear safety analysis and design, Version 6.1, Oak Ridge National Laboratory, 2011.

10. J. Leppänen, Private communication, 2014.

11. R. J. Ellis, et al., "Generation of a broad-Group HTGR library for use with SCALE," NUREG/CR-7106, ORNL/TM-2011/298, 2012, p. 8.

12. M. Ouisloumen, R. Sanchez, "A model for neutron scattering off heavy isotopes that accounts for thermal agitation effects," Nuclear Science and Engineering, Vol. 107, No. 3, March 1, 1991, pp. 189-200.

13. M. L. Williams, "Resonance self-shielding methodologies in SCALE 6," Nuclear Technology, Vol. 174, No. 2, May 2011, pp. 149-168.

14. M. Goto, et al., "Impact of revised thermal neutron capture cross section of carbon stored in JENDL-4.0 on HTTR criticality calculation," Journal of Nuclear Science and Technology, Vol. 48, No. 7, January 5, 2011 pp. 965-969.

15. J. Kim, G Kim, C. Huh, "Assessment of double heterogeneity treatment capability in SCALE," Proceedings of the 22nd International Conference Nuclear Energy for New Europe, Bled, Slovenia, September 9-12, 2013, pp. 613.1-613.8.

16. J.-Y. Wang, et al., "HTTR criticality calculations with SCALE6: Studies of various geometric and unit-cell options in modeling," Proceedings of PHYSOR 2012, Knoxville, U.S.A., April 15-20, 2012. 
17. R. W. Swanson, L. J. Harrison, "The effect of carbon crystal structure on treat reactor physics calculations," CONF-880911-23, DE89 003625, International Reactor Physics Conference, Jackson Hole, U.S.A., September 18-22, 1988, Vol. 4.

18. A. I. Hawari, V. H. Gillete, "Inelastic thermal neutron scattering cross sections for reactor-grade graphite," Nuclear Data Sheets, Vol. 118, April 2014, pp. 176-178.

19. J. Leppänen, M. DeHart, "HTGR reactor physics and burnup calculations using the Serpent Monte Carlo code," American Nuclear Society, Vol. 101, January 2009, pp. 782-784.

20. J. Žáková, A. Talamo, "Criticality assessment for prismatic high temperature reactors by fuel stochastic Monte Carlo modeling," Annals of Nuclear Energy, Vol. 35, No. 5, May 2008, pp. 856860 .

21. M.-H. Chiang, et al., "Evaluation of the HTTR criticality and burnup calculations with continuous-energy and multigroup cross sections," Nuclear Engineering and Design, Vol. 271, May 2014, pp. 327-331.

22. D. Ilas, J. Gehin, "HTTR fuel block simulations with SCALE," Proceedings of PHYSOR-2010, Pittsburgh, USA, May 9-14, 2010.

23. NEA, "Temperature effect on reactivity in VHTRC-1 core," VHTRC-GCR-EXP-001, CRIT-COEF, NEA/NSC/DOC(2006)2, 2006.

24. LANL, "MCNP - A General Monte Carlo N-Particle Transport Code, Version 5, Volume I: Overview and Theory," LA-UR-03-1987, Los Alamos National Laboratory, 2003.

25. ANSYS Inc., “ANSYS CFX-Solver Theory Guide,” ANSYS Inc., Canonsburg, Pennsylvania, November, 2009.

26. S.-J. Yoon, G. Strydom, "Comparison of Homogeneous and Heterogeneous CFD Models for Phase I of the IAEA CRP on HTGR Uncertainties Benchmark," Proceedings of PHYSOR 2014, Charlotte, U.S.A., April 6-9, 2014.

27. B. S. Petukhov, "Heat transfer and friction in turbulent pipe flow with variable physical properties," Advances in Heat Transfer, Vol. 6, pp., 1970, 503-565.

28. F. Incropera and D. DeWitt, 2007, Fundamentals of Heat and Mass Transfer, 6th ed., Wiley, New York.

29. K. B. Bekar, et al., "Enhancements in Continuous-Energy Monte Carlo Capabilities in SCALE," Proceedings of ANS NCSD 2013 - Criticality Safety in the Modern Era: Raising the Bar, Wilmington, North Carolina, September 29-October 3, 2013.

30. K. B. Bekar, et al., "Enhancements in Continuous-Energy Monte Carlo Capabilities in SCALE," Presentation of ANS NCSD 2013, Wilmington, NC, September 29-October 3, 2013.

31. B. T. Rearden, et al., "Overview of SCALE 6.2," Proceedings of ANS NCSD 2013, Wilmington, North Carolina, September 29-October 3, 2013.

32. F. B. Brown, "On the Use of Shannon Entropy of the Fission Distribution for Assessing Convergence of Monte Carlo Criticality Calculations," Proceedings of PHYSOR-2006, ANS Topical Meeting on Reactor Physics, Vancouver, Canada, September 10-14, 2006.

33. Y. Kim, K.-S. Kim, J. Man Noh, "Reactivity-Equivalent Physical Transformation for Homogenization of Double-Heterogeneous Fuels," Transactions of the Korean Nuclear Society, Autumn Meeting, Busan Korea, October 27-28, 2005. 


\section{APPENDIX A}

\section{ADDITIONAL INVESTIGATIONS ON NEWT RESULTS, ALTERNATIVE METHODS AND LIBRARY STRUCTURES}

\subsection{SCALE/NEWT Criticality Calculations with 238 Groups}

As a preparation for the SCALE/SAMPLER calculations, NEWT inputs for the MHTGR have been compiled. NEWT is a deterministic code and requires two-dimensional inputs. For the fuel compacts the same MG cells as in the KENO calculations of the reference results are applied. The burnable poison compacts have been homogenized in order to avoid a particle structure.

The multiplication factors of these calculations with SCALE 6.1.2 are presented in Table 29. The values are furthermore compared to the corresponding KENO MG calculation, (e.g., for Exercise I-2a the values are compared with KENO MG calculations including homogeneous burnable poison compacts). All results included here used the 238-group structure. The input for the super cell of Exercise I-2c could not be successfully compiled at this stage.

For all calculations, 6 azimuthal and 3 polar angles were chosen. The fuel compact unit cells consisted of a $4 \times 4$ grid, all other compacts of a $2 \times 2$ grid. Furthermore the coarse-mesh finite-difference acceleration has been applied. The PN order for scattering in the material mixtures is set to 1 for the homogeneous fuel compact, and to 2 in all other (DOUBLEHET) cases.

The NEWT calculations for Exercise I-1a show multiplication factors within the standard deviation of the respective KENO MG results, and the DOUBLEHET calculations of Exercise I-1b show only small differences below $60 \mathrm{pcm}$ to the KENO results. The fresh fuel block (Exercise I-2a) showed an underestimation of the multiplication factor of more than $300 \mathrm{pcm}$, whereas the depleted fuel block is within the standard deviation of KENO. Apart from different fuel compositions, the only other difference between the fuel block models is the consideration of burnable poison compacts in the fresh block. The addition of BP compacts is therefore suggested to be the cause of the deviation from the KENO result. A change of, for example, the unit cell grid or the angular resolution might improve the result. Further investigation into the reaction rates and spectra would also be necessary to complete this preliminary assessment.

The differences between NEWT and KENO MG multiplication factors are relatively small, and the use of the NEWT seem to be a possible option for accurate multi-group calculations with a lower calculation burden.

Table 29. Comparison of the NEWT and SCALE 6.1.2 KENO MG (238 groups) multiplication factors.

\begin{tabular}{|l|c|c|c|c|}
\hline & $\mathbf{C Z P}$ & $\begin{array}{c}\Delta \\
{[\mathbf{p c m}]}\end{array}$ & HFP & $\begin{array}{c}\Delta \\
\text { [pcm] }\end{array}$ \\
\hline Exercise I-1a & 1.25412 & -3 & 1.18299 & -1 \\
\hline Exercise I-1b & 1.31070 & -42 & 1.24282 & -57 \\
\hline Exercise I-2a & - & - & 1.04871 & -315 \\
\hline Exercise I-2b & - & - & 0.96903 & -10 \\
\hline
\end{tabular}




\subsection{REACTIVITY-EQUIVALENT Physical Transformation of the MHTGR-350}

Kim et al. ${ }^{33}$ suggested a reactivity-equivalent physical transformation (RPT) for the homogenization of double-heterogeneous fuel. As observed in the reference results of Exercise I-1a, a homogenization of the fuel compact cylinder leads to a significant underestimation of the multiplication factor due to reduction in self-shielding of the particles. The RPT method suggests moving the particles into a homogenized cylinder with a smaller radius. The space between the cylinder and the original fuel radius is filled with graphite matrix. The radius of the new cylinder can be obtained by iteration calculations in order to match the reference multiplication factor of a corresponding double-heterogeneous calculation.

For the calculations in this limited comparison, Serpent 2 was again applied. The reference value was the Serpent calculation performed for Exercise I-1b that included a random particle distribution. The ENDF-B-VII.0 library was applied. The obtained multiplication factors and the reduced radius for the RPT method are presented in Table 30. The RPT ko are within the standard deviation of the reference. The neutron flux spectra for the HFP state are presented in Figure 56. The difference between the spectra is less than $0.001 \%$. It is especially noteworthy that the resonances of the homogenized RPT model show very similar resonances to the double-heterogeneous model. In comparison to Exercise I-1a, the reduced RPT radius enhances the fuel self-shielding.

Table 30. RPT values of the fuel compact unit cell (Exercise I-1b).

\begin{tabular}{|l|c|c|}
\hline & CZP & HFP \\
\hline Reference $\mathrm{k}_{\infty}$ & $1.31865 \pm 0.00012$ & $1.24657 \pm 0.00013$ \\
\hline RPT radius [cm / \% of original] & $0.47159 / 76$ & 0.47174 \\
\hline RPT $\mathrm{k}_{\infty}$ & $1.31887 \pm 0.00011$ & $1.24655 \pm 0.00012$ \\
\hline
\end{tabular}

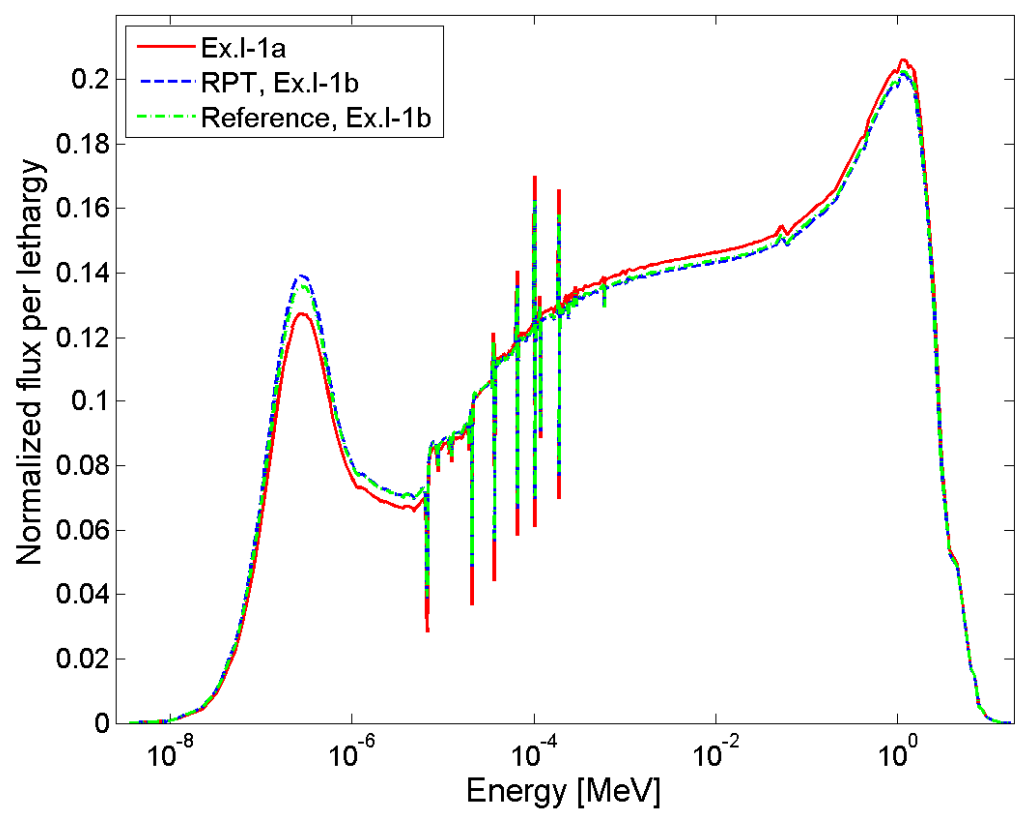

Figure 56. Normalized neutron flux spectrum of the reference heterogeneous Serpent calculation and the homogeneous RPT method (Exercise I-1b, HFP). 
In a subsequent test, it was investigated if the obtained RPT radius of the Exercise I- $1 \mathrm{~b}$ HFP state can also be utilized into the next exercise, [i.e., a fresh fuel block (Exercise I-2a)]. However, differences of several hundred pcm are obtained for both a fresh block with and without burnable poison (see Table 31). The use of the reduced radius homogenous model for the super cell (Exercise I-2c) did however result in a much smaller difference of $55 \mathrm{pcm}$ compared to the reference. The influence of the fuel compact model decreases in the super cell, as already observed in the discussion of the reference results. The differences of the fluxes in the homogeneous and heterogeneous super cells are again below $0.001 \%$.

Table 31. RPT $\mathrm{k}_{\infty}$ of Exercise I-2a/c compared to the corresponding Serpent calculation.

\begin{tabular}{|c|c|c|}
\hline & $\mathbf{k}_{\infty} \pm \boldsymbol{\sigma}$ & $\begin{array}{c}\Delta \pm \sigma \\
{[\mathrm{pcm}]}\end{array}$ \\
\hline Exercise I-2a with BP & $1.05799 \pm 0.00008$ & $-505 \pm 12$ \\
\hline Exercise I-2a without BP & $1.38522 \pm 0.00006$ & $-725 \pm 9$ \\
\hline Exercise I-2c: $\mathrm{k}_{\infty}$ & $1.04955 \pm 0.00005$ & $-55 \pm 7$ \\
\hline
\end{tabular}

Although this investigation was limited in scope, it therefore seems that the RPT method might be worth further investigation, especially if the participants' code for uncertainty calculations is not able to include double-heterogeneous fuel models. An interesting comparison might be the uncertainty calculation comparison using SCALE/TSUNAMI MG of a RPT equivalent model for Exercise I-1b against the TSUNAMI results for Exercise I-1a. These results can be cross-checked with the new TSUNAMI-KENO CE predictions available in SCALE 6.2, where a double heterogeneous treatment is not required, and also the stochastic predictions of the SAMPLER module.

It is however recommended that the validity of this method be validated against the VHTRC experimental data set, before the RPT approach is applied to the CRP exercises. It might also be the case that the RPT method performs well where integral parameters are concerned (total flux, multiplication factor), but that larger differences exist in local reaction rates.

\subsection{SCALE/KENO Calculations Using the 56-Group Library}

In addition to the 238- and 252-group calculations with SCALE/KENO 6.2b3, calculations with the new (pre-compiled) 56 energy group library with have also been performed. The results are presented in Table 32, and also compared to the respective 238-group calculation with SCALE 6.2b3.

The use of this library led to a k $\infty$ underestimation of the 238-group results by several hundred pcm for all exercises. This group structure therefore seems not to be suitable for graphite systems, which is not surprising as this library is optimized for LWR applications. The 56-group library calculation times were also longer. It has to be mentioned that this 56-group structure is so far only a prototype library, however, and the relative performance might change until the final release. At this point, this library option cannot be recommended for HTGR applications. 
Table 32. Multiplication factor of SCALE/KENO $6.2 \mathrm{~b} 3$ calculations with 56 energy groups compared to the respective 238-group calculations.

\begin{tabular}{|l|c|c|c|c|}
\hline & \multicolumn{2}{|c|}{ CZP } & \multicolumn{2}{c|}{ HFP } \\
\hline & & $\begin{array}{c}\Delta \pm \boldsymbol{\sigma} \\
\text { [pcm] }\end{array}$ & $\mathbf{k}_{\infty} \pm \boldsymbol{\sigma}$ & $\begin{array}{c}\Delta \pm \boldsymbol{\sigma} \\
\text { [pcm] }\end{array}$ \\
\hline Exercise I-1a & $1.24683 \pm 0.00016$ & $-737 \pm 22$ & $1.17702 \pm 0.00015$ & $-597 \pm 21$ \\
\hline Exercise I-1b & $1.30658 \pm 0.00014$ & $-644 \pm 20$ & $1.23912 \pm 0.00014$ & $-581 \pm 20$ \\
\hline Exercise I-2a & - & - & $1.06626 \pm 0.00015$ & $-435 \pm 21$ \\
\hline Exercise I-2b & - & - & $0.96203 \pm 0.00011$ & $-822 \pm 16$ \\
\hline Exercise I-2c & - & - & $1.04789 \pm 0.00013$ & $-540 \pm 19$ \\
\hline
\end{tabular}

\subsection{SCALE/KENO and SCALE/NEWT Calculations Using an ORNL 81-Group Structure}

Ellis et al. ${ }^{11}$ suggested an optimized 81-group structure for HTGR analyses. Initial work was performed at ORNL, but the library was not included in any SCALE release so far, and no plans exist at ORNL to include it in future SCALE releases as a standard library. However, as a SCALE/NEWT user option, an 81-group library can be created by collapsing the 238-group master library into a user-defined 81 group structure. This library is flux-weighted with the 238 group solution for this specific CRP problem.

As a first assessment on the performance of this group structure for the CRP problem set, 81-group libraries were created for Exercise I-1a/b and I-2a/b and afterwards utilized by both NEWT and KENO, using SCALE Version 6.1.2. The resulting multiplication factors are compared in Table 33 and Table 34 with the respective 238-group calculations. Table 33 also includes a comparison of the relative run-times required for these calculations. The input for the super cell of Exercise I-2c could not be successfully compiled at this stage.

Both the NEWT and the KENO results using the new 81-group library show excellent agreement with the 238-group calculations. The differences in the set of NEWT calculations are less than $20 \mathrm{pcm}$, and the calculation time is decreased by more than a factor of two. The KENO calculations of the new group structure have overlapping error bars with the 238-group calculations.

These results look very promising, especially with regard to the reduction in calculation time with NEWT. It might be sufficient for subsequent uncertainty calculations to utilize the new 81-group structure (as opposed to a more expensive 238-group library), but a more detailed comparison including flux spectra and reaction rates should be performed prior to the use of this group structure in future calculations.

Table 33. Multiplication factor of SCALE/NEWT 6.1.2 calculations with 81 energy groups compared to the respective 238-group calculations.

\begin{tabular}{|l|c|c|c|c|c|c|}
\hline & \multicolumn{3}{|c|}{ CZP } & \multicolumn{3}{c|}{ HFP } \\
\hline & $\mathbf{k}_{\infty}$ & $\begin{array}{c}\Delta \\
{[\mathbf{p c m}]}\end{array}$ & $\begin{array}{c}\text { Duration } \\
\mathbf{8 1 g} / \mathbf{2 3 8 g}\end{array}$ & $\mathbf{k}_{\infty}$ & $\begin{array}{c}\Delta \\
{[\mathbf{p c m}]}\end{array}$ & $\begin{array}{c}\text { Duration } \\
\mathbf{8 1 9} / \mathbf{2 3 8 g}\end{array}$ \\
\hline Exercise I-1a & 1.25424 & 12 & $40 \%$ & 1.18318 & 19 & $36 \%$ \\
\hline Exercise I-1b & 1.31075 & 5 & $39 \%$ & 1.24292 & 10 & $37 \%$ \\
\hline Exercise I-2a & - & - & - & 1.04876 & 5 & $36 \%$ \\
\hline Exercise I-2b & - & - & - & 0.96910 & 7 & $28 \%$ \\
\hline
\end{tabular}


Table 34. Multiplication factor of SCALE/KENO 6.1.2 calculations with 81 energy groups compared to the respective 238 -group calculations.

\begin{tabular}{|l|c|c|c|c|}
\hline & \multicolumn{2}{|c|}{ CZP } & \multicolumn{2}{c|}{ HFP } \\
\hline & $\mathbf{k}_{\infty} \pm \boldsymbol{\sigma}$ & $\begin{array}{c}\Delta \pm \boldsymbol{\sigma} \\
{[\mathbf{p c m}]}\end{array}$ & $\mathbf{k}_{\infty} \pm \boldsymbol{\sigma}$ & $\begin{array}{c}\Delta \pm \boldsymbol{\sigma} \\
\text { [pcm] }\end{array}$ \\
\hline Exercise I-1a & $1.25426 \pm 0.00015$ & $11 \pm 21$ & $1.18327 \pm 0.00013$ & $27 \pm 20$ \\
\hline Exercise I-1b & $1.31133 \pm 0.00013$ & $21 \pm 19$ & $1.24341 \pm 0.00013$ & $2 \pm 20$ \\
\hline Exercise I-2a & - & - & $1.06945 \pm 0.00015$ & $0 \pm 21$ \\
\hline Exercise I-2b & - & - & $0.96921 \pm 0.00012$ & $8 \pm 16$ \\
\hline
\end{tabular}

